\title{
WestVirginiaUniversity
}

THE RESEARCH REPOSITORY @ WVU

Graduate Theses, Dissertations, and Problem Reports

2017

\section{Investigating stress response across integrated biological systems}

Alice A. Han

Follow this and additional works at: https://researchrepository.wvu.edu/etd

Part of the Analytical Chemistry Commons, and the Other Chemistry Commons

\section{Recommended Citation}

Han, Alice A., "Investigating stress response across integrated biological systems" (2017). Graduate Theses, Dissertations, and Problem Reports. 5748.

https://researchrepository.wvu.edu/etd/5748

This Dissertation is protected by copyright and/or related rights. It has been brought to you by the The Research Repository @ WVU with permission from the rights-holder(s). You are free to use this Dissertation in any way that is permitted by the copyright and related rights legislation that applies to your use. For other uses you must obtain permission from the rights-holder(s) directly, unless additional rights are indicated by a Creative Commons license in the record and/ or on the work itself. This Dissertation has been accepted for inclusion in WVU Graduate Theses, Dissertations, and Problem Reports collection by an authorized administrator of The Research Repository @ WVU.

For more information, please contact researchrepository@mail.wvu.edu. 


\title{
Investigating stress response across integrated biological systems
}

\author{
Alice A. Han \\ Dissertation submitted to the \\ Eberly College of Arts and Sciences \\ at West Virginia University
}

in partial fulfillment of the requirements for the degree of

Doctor of Philosophy in

Chemistry

\author{
Jonathan W. Boyd, Ph.D., Chair \\ Harry O. Finklea, Ph.D. \\ John B. Mertz, Ph.D. \\ Stephen J. Valentine, Ph.D. \\ James P. O'Callaghan, Ph.D. \\ C. Eugene Bennett Department of Chemistry
}

Morgantown, West Virginia
2017

Keywords: Stress Response, Cytokines, Phosphoprotein, Signaling, Network Centrality Parameter Analysis

Copyright 2017 Alice A. Han 


\begin{abstract}
Investigating stress response across integrated biological systems
\end{abstract}

\begin{abstract}
Alice A. Han
The impact that a stressor has on a living system, whether it is on a cellular, organ, or even a whole organism level, can affect the overall health of the system. Monitoring the biochemical response resulting from a stressful experience offers insight into the numerous potential outcomes ranging on the spectrum of survival and death. Accessing this information not only provides a heightened understanding of the biochemical adaptions that occur, but also allows for the development of prediction models (to assess prospective influences of the stressor) or potential therapeutic treatments (to alleviate adverse effects) using measurable, quantifiable, and comparable metrics. It is, however, a continuing effort to decipher the results as these detected responses can be complex, and furthermore extremely context specific. Biochemical patterns can be biased by the many discrepancies in the types, degrees, and frequencies of stressors, time points at which the responses are measured, the biological matrix that is assessed, and even the selected cohort of targets. The work of this dissertation observed intracellular (i.e. protein expression, phosphorylation modifications), extracellular (i.e. cytokine), and hormonal (cortisol, ACTH) responses, following exposures to a variety of physical, social, and environmental stressors. A series of statistical treatments, including network centrality parameter analyses, were implemented to dissect these complex datasets. The findings suggested that the changes in the measured biochemical responses - triggered by certain stressors - could be distinguished among different degrees of experienced stress, as well as different time points of measurement. The overarching objective of this research and of all future related research is to bring insight into the complex biological response system and demonstrate how particular stressors can prompt discrete trends. Overall, there appears to be potential value in monitoring biochemical alterations to describe the stress response.
\end{abstract}




\section{DEDICATION}

This dissertation is dedicated to my parents - Chong Mo and Young Hee Han and my sister - Erica Han for their unconditional love, support, and patience. 


\section{ACKNOWLEDGEMENTS}

First and foremost, I would like to acknowledge and thank my advisor and mentor, Dr. Jonathan Boyd. I don't think I could ever fully express how much I appreciate everything you've given me. Beyond training me to become a better investigator, you've instilled in me the importance of perspective, compassion, and having fun with research. Thank you for all your hard work over the past 5 years.

While I dedicate this work to my family, they also deserve so much recognition. Mom, Dad, Erica - thank you for being so patient with me even when I didn't really deserve it. I often forget about the sacrifices everyone has made for me and I can only hope to make it up to you guys someday. All I want to do is make everyone proud and I will work hard every day to be the best version of myself. I don't say this enough because you know I don't do touchy-feely emotion things, but I love all of you. Now it's in writing forever :)

To my former lab mates, Dr. Julie Miller and Dr. Holly Currie: I am so happy for you two for all your achievements and success! In joining the Boyd research group, I not only found great scientists to look up to, but great friends as well. Thank you for teaching me the tricks of surviving graduate school, supporting me through all my times of struggle, and for giving me so many fun memories. While we are all in different places, I hope to always maintain our friendship.

To my fellow lab mates, Nicole Prince, Julia Mouch, and Maren Prediger: Even though we haven't worked together for very long, it doesn't seem like it at all. I've had so much fun this past

year - both research and non-research related. I still can't believe we collected Zephyr data at iv 
Fright Farm and in the Escape Room (I expect the Boyd group to continue with this "research" and please send me data!). I wish everyone the best and know that I will always be available to help.

To all my friends that kept me sane throughout the years: Emily Fabyanic, Michael Spencer, Bill Feeney, Julie Miller, Holly Currie, Nicole Prince, Julia Mouch, Rob Crovak, Reem Eldawud, Corey Nida, Albert Tomasso, Zach Sasiene, Emily Despeaux and Liz Ward. I feel incredibly lucky to have met all of you and we BETTER keep in touch...

To Dr. Kimberly Quedado and Dr. Vincent Castranova: My graduate training would have been so different without the IGERT fellowship. I feel so privileged to have had access to the resources and opportunities provided through this fellowship. Thank you so much for putting in the work to oversee a bunch of stubborn, outspoken, and opinionated students and genuinely caring for our success.

Last, and certainly not least, my committee members, Dr. James O'Callaghan, Dr. Harry Finklea, Dr. Blake Mertz, Dr. Stephen Valentine: thank you for the encouragement, patience, and guidance throughout my graduate career. Honorable mention goes to my former committee member, Dr. Kenneth Blemings, for sitting through my candidacy defense. 


\section{TABLE OF CONTENTS}

$\begin{array}{ll}\text { Title Page } & \text { i }\end{array}$

$\begin{array}{ll}\text { Abstract } & \text { ii }\end{array}$

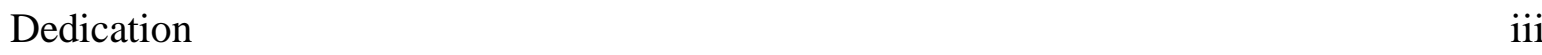

Acknowledgements $\quad$ iv

Table of Contents

List of Figures $\quad$ xii

List of Tables $\quad$ xiv

Chapter 1: Introduction to stressors and measurable biological responses ...............................1

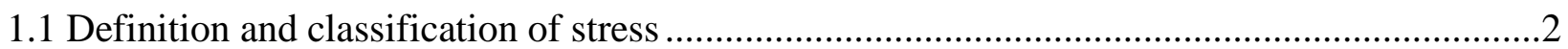

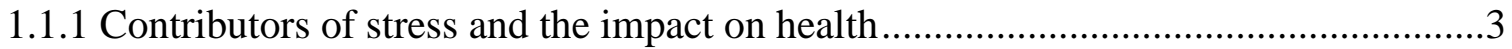

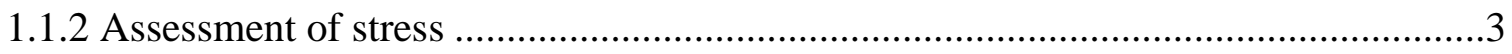

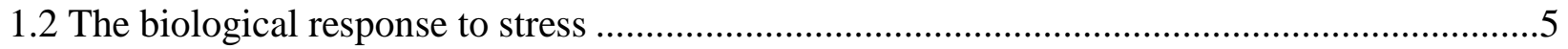

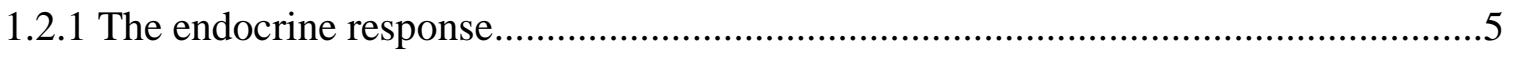

1.2.1.1 The hypothalamic-pituitary-adrenal axis .........................................6

1.2.1.2 The sympathetic-adrenal-medullary system .......................................

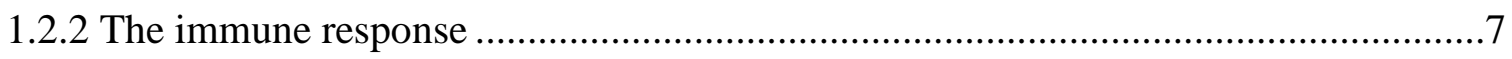

1.2.2.1 Relating the immune response the stress ........................................

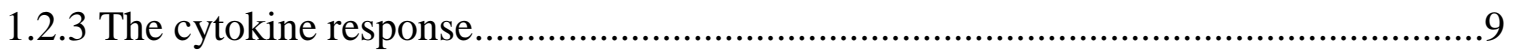

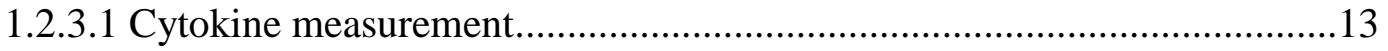


1.2.3.2 Cytokines and stress................................................................... 14

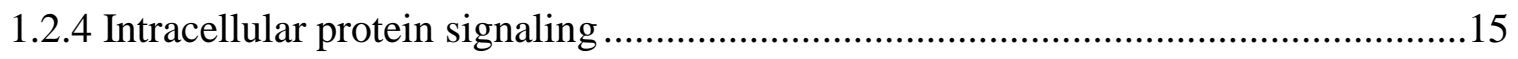

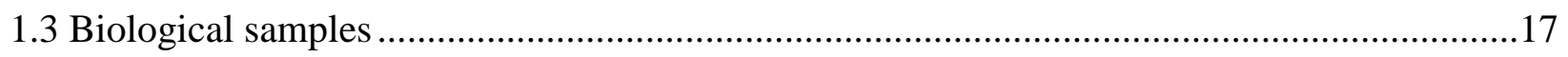

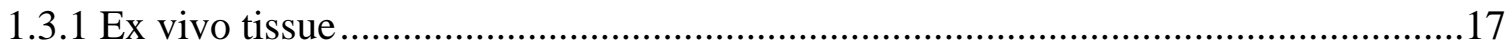

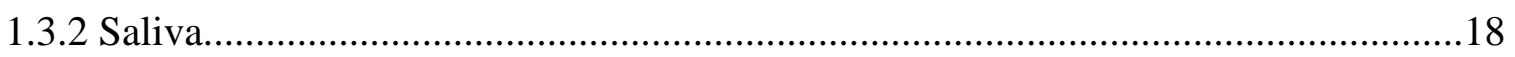

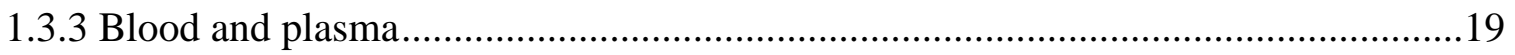

1.4 Bioinformatics: analyzing complex biological datasets .............................................. 19

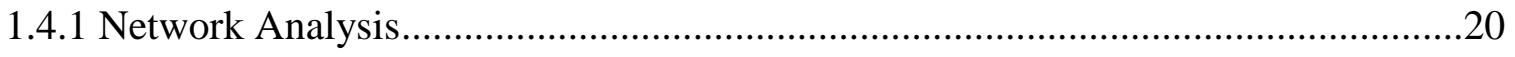

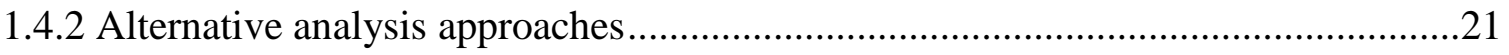

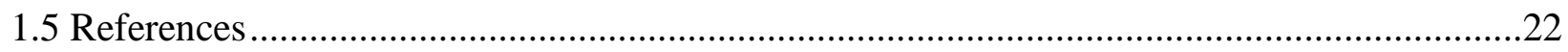

\section{Chapter 2: Spatiotemporal phosphoprotein distribution and} associated cytokine response of a traumatic injury ............................................31

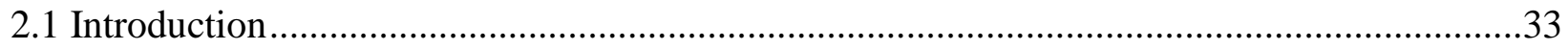

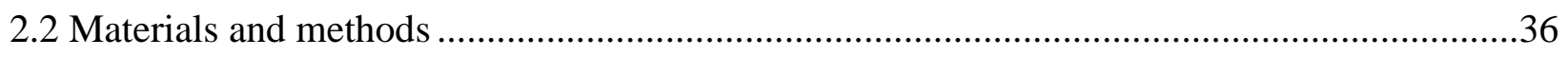

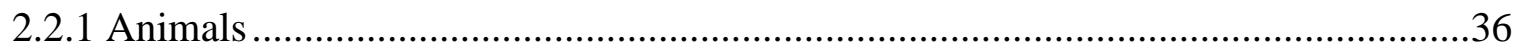

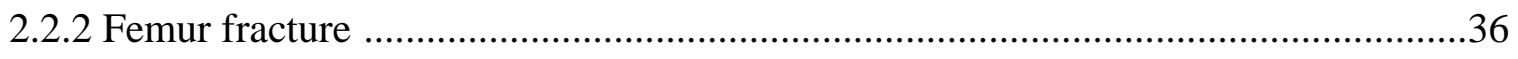

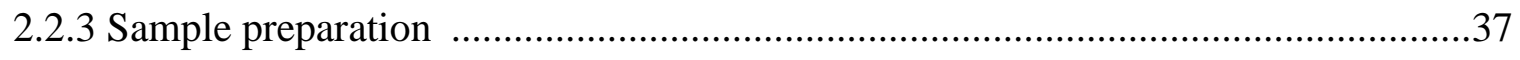

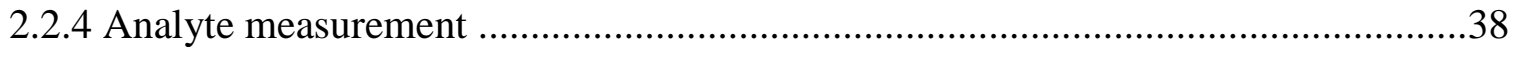

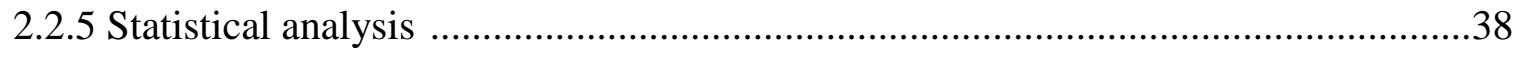

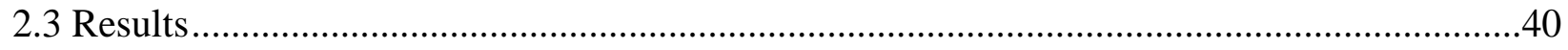


2.3.1 Spatial and temporal total protein levels................................................................40

2.3.2 Spatial and temporal response of phosphorylated proteins.......................................41

2.3.3 Spatial and temporal cytokine concentrations .......................................................4

2.3.4 Quadratic discriminant analysis used to predict location and time of injury............46

2.3.5 Correlation between cytokine and phosphoprotein response....................................49

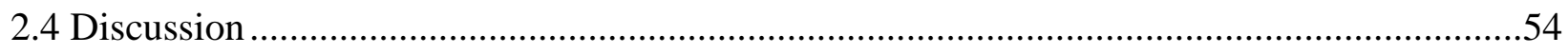

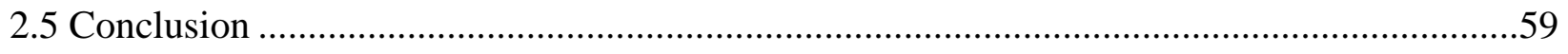

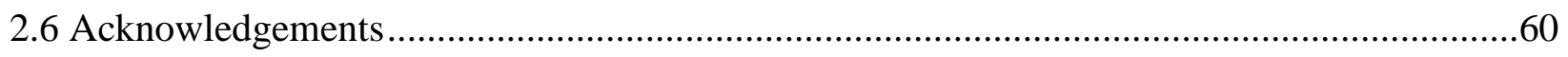

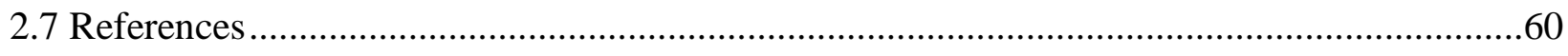

Chapter 3: Using network centrality parameter analysis to investigate

intra- and extracellular signaling responses of a traumatic injury ........................66

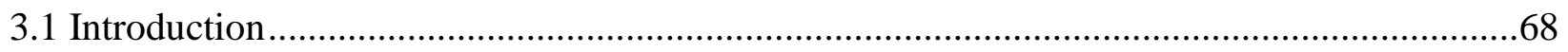

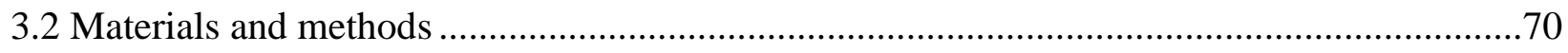

3.2.1 Data description ............................................................................................

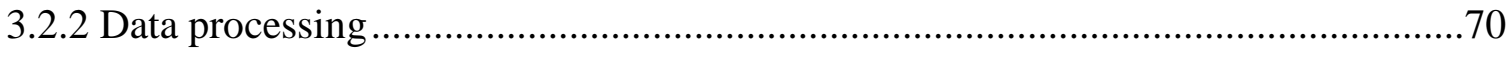

3.2.3 Network evaluation with Ingenuity Pathway Analysis ...........................................71

3.2.4 Network centrality parameter analysis ................................................................73

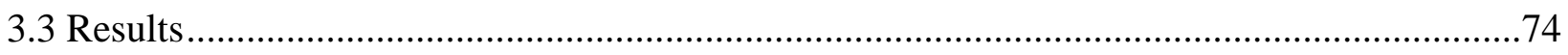

3.3.1 Relative spatial and temporal cytokine response ……............................................74

3.3.2 Relative spatial and temporal protein response ………………..............................74 
3.3.3 Relative spatial and temporal response of phosphorylated proteins

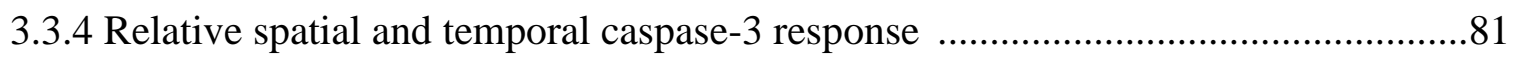

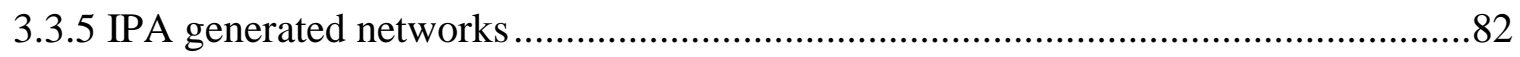

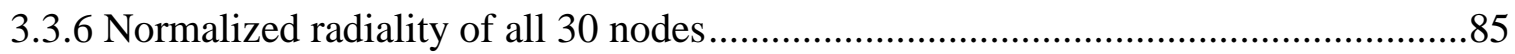

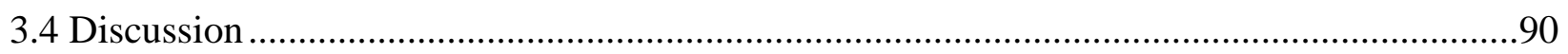

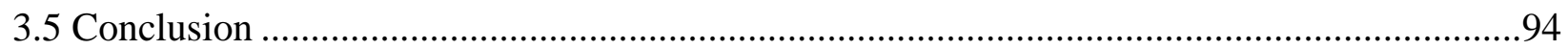

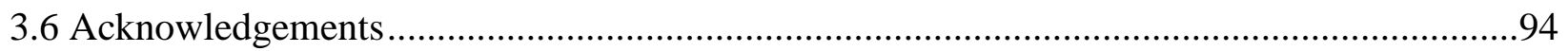

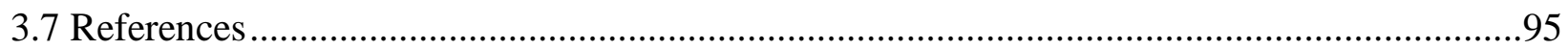

Chapter 4: The investigation of salivary cytokine response to individual and shared success

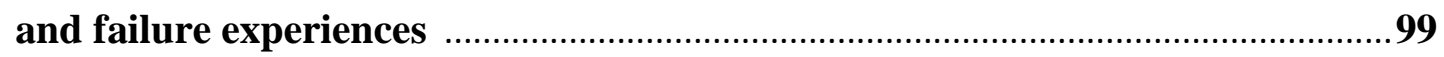

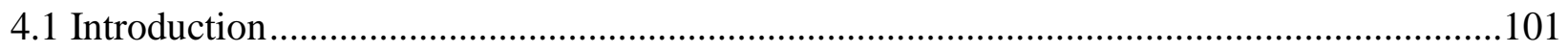

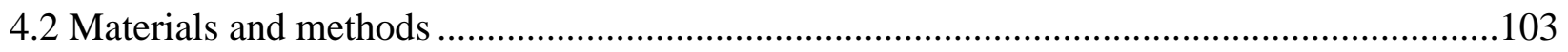

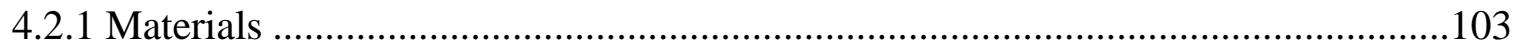

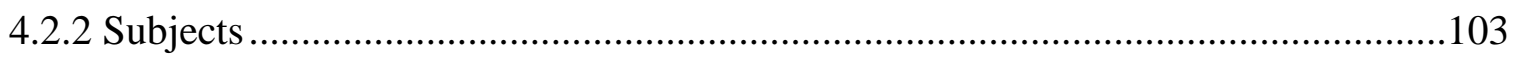

4.2.3 Experimental design: mock hostage rescue ………............................................104

4.2.4 Saliva sample collection and analysis ............................................................106

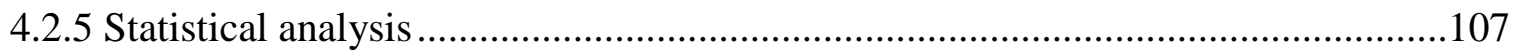

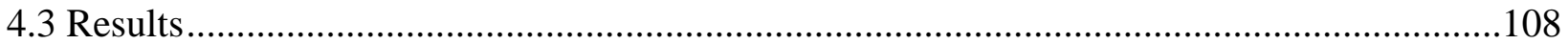

4.3.1 Normalized salivary cytokine responses of individual and team performances .....................................................................108 
4.3.2 Task completion times ...................................................................................110

4.3.3 Principal component analysis score and loading plots ..........................................111

4.3.4 Hierarchical cluster analysis with Ward's minimum variance ................................113

4.3.5 Baseline cytokine concentrations ..................................................................114

4.3.6 Success and failure associated salivary cortisol concentrations ............................114

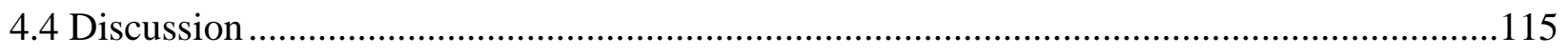

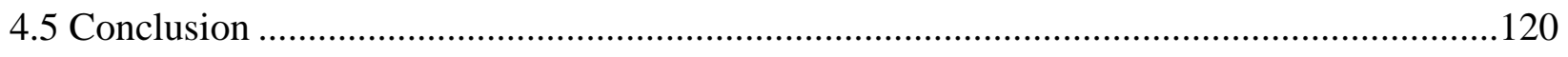

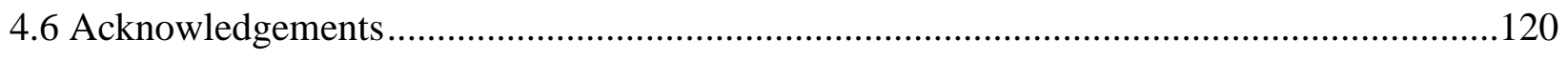

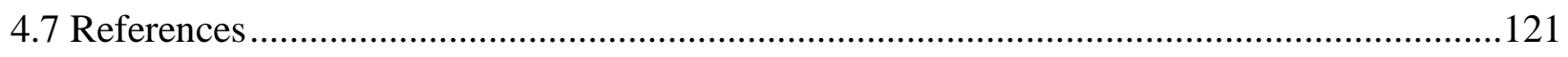

Chapter 5: Identification of Biomarkers Related to Perceived Stress Scale-10 and the Evaluation of the Survey Components ...........................................................127

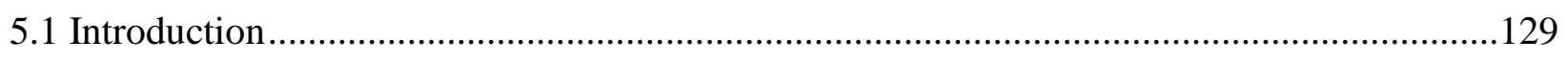

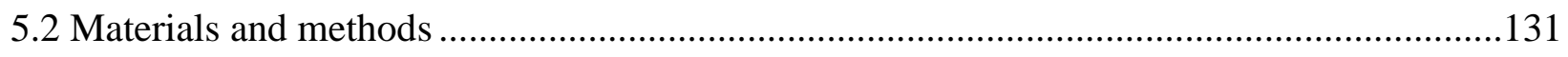

5.2.1 Subjects ..................................................................................................131

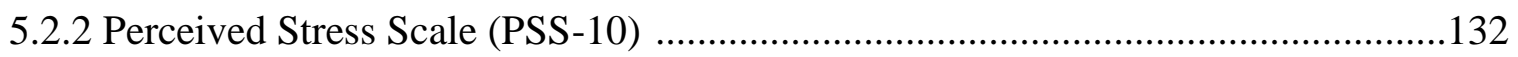

5.2.3 Plasma sample preparation and analysis ...........................................................134

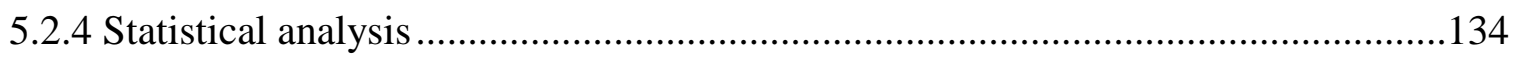

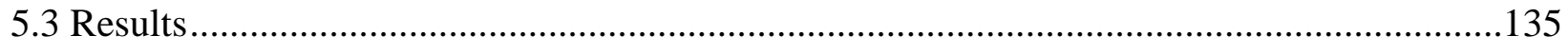

5.3.1 Biomarkers of overall PSS-10 scores ………………...................................135

5.3.2 Biomarkers of PSS-10 question 2 (Q2) scores ................................................136 
5.3.3 Biomarkers of PSS-10 question 5 (Q5) scores

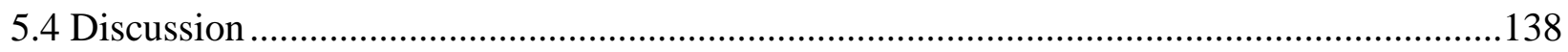

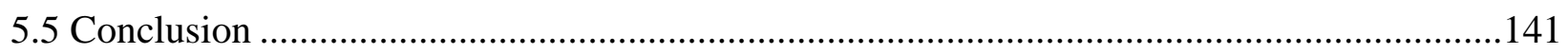

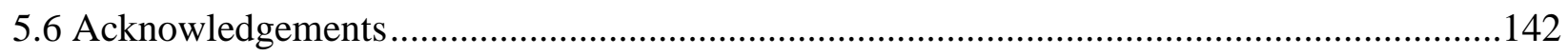

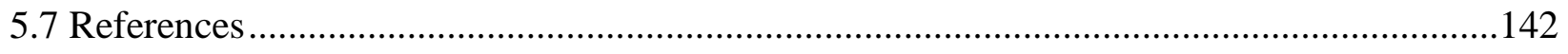

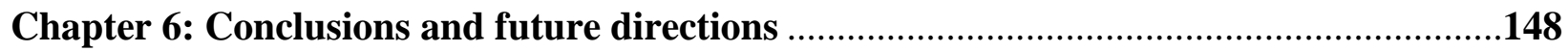

Appendix A: Cytokine Calibration Curves .....................................................................................151

Appendix B: Chapter 4 supplementary figures .................................................................................152 


\section{LIST OF FIGURES}

Figure 2.1. Total protein concentration measured across time and location in response to a traumatic injury

Figure 2.2. Phosphorylation levels measured across time and location in response to a traumatic injury

Figure 2.3. Phosphorylation levels measured across time and location in response to a traumatic injury

Figure 2.4 Cytokine levels measured across time and location in response to a traumatic injury

Figure 2.5. Canonical scores plot for the identification of the location of injury

Figure 2.6. Canonical scores plot for the identification of the time of injury

Figure 3.1. Relative cytokine levels measured across time and injury location in muscle tissue resulting from a femur fracture

Figure 3.2. Relative protein response measured across time and injury location in muscle tissue resulting from a femur fracture

Figure 3.3. Relative phosphorylation response measured across time and injury location in muscle tissue resulting from a femur fracture

Figure 3.4. Relative phosphorylation response measured across time and injury location in muscle tissue resulting from a femur fracture

Figure 3.5. Relative Caspase- 3 activity measured across time and injury location in muscle tissue resulting from a femur fracture

Figures 3.6. (top) and 3.7. (bottom) IPA generated networks constructed from protein, cytokine, phosphoprotein, and protease dataset

Figure 3.8. Significant radiality outcomes associated with responses at the site of fracture

Figure 3.9. Significant radiality outcomes associated with responses 1-cm away from the site of fracture

Figure 4.1. Normalized cytokine response following team and individual 
Figure 4.2. (top) and 4.3. (bottom). PCA: score and loading plots of cytokine response

Figure 4.4. (left) and 4.5. (right). Hierarchical cluster analysis with Ward's minimum variance

Figure 5.1. Plasma ACTH levels of high and low perceived stress subjects

Figure 5.2. Plasma cortisol levels of high and low perceived stress subjects - Q2

Figure 5.3. Plasma ACTH levels of high and low perceived stress subjects - Q5

Figure 5.4. Plasma insulin levels of high and low perceived stress subjects - Q7

Figure 5.5. Plasma insulin levels of high and low perceived stress subjects - Q9

Figure A1. Cytokine Calibration Curves

Figure B1. Subjects 1-4: Normalized cytokine response following team and individual performance

Figure B2. Subjects 5-8: Normalized cytokine response following team and individual performance

Figure B3. Subjects 9-12: Normalized cytokine response following team and individual performance

Figure B4. Subjects 13-14: Normalized cytokine response following team and individual performance 


\section{LIST OF TABLES}

Table 2.1. Results of spatial cytokine-total protein response correlation analysis

Table 2.2. Results of spatial cytokine-phosphorylated protein response correlation analysis

Table 2.3. Results of temporal cytokine-total protein response correlation analysis

Table 2.4. Results of temporal cytokine-phosphorylated protein response correlation analysis

Table 3.1. Intra and Extracellular biomolecules measured after traumatic injury

$\begin{array}{ll}\text { Table 3.2. Top IPA Networks } & 84\end{array}$

Table 3.3. Normalized radiality of nodes at the site of injury 86

Table 3.4. Normalized radiality of nodes 1-cm away from injury $\quad 87$

Table 4.1. Subject Information 104

Table 4.2. Task completion times 110

Table 4.3. Averaged baseline cytokine concentrations 114

Table 4.4. Averaged salivary cortisol concentrations 115

Table 5.1. 10 questions of the PSS-10 survey supplied to the subjects 132

Table 5.2. Profiles of each subject 


\section{Chapter 1}

\section{Introduction to stressors and measurable biochemical responses}




\subsection{Definition and classifications of stress}

As research has progressed, the definition and organization of the different types of stress that living systems can experience has expanded and evolved. In the most basic terms, stress is broadly used to describe the resulting changes produced by a stressor, or an external force imposed on a living entity $[1,2]$. Considering stress can encompass numerous influences, it is critical to be aware of, and identify, all of the potential factors, especially when it comes to investigating the stress response. These varying factors can include types of stressors (e.g. external, internal, distress, eustress), duration and frequency of exposure, number of stressors experienced at one time, new versus pre-existing stressors, as well as the profile of the subject in question (sex, age, health, organ systems and even the type of cell).

It is common to associate stress with a negative connotation, but stressors can also prompt positive effects. In fact, distress is known as the consequence of the demands surpassing the capacity to maintain homeostasis, while eustress describes the optimal level of stress that is beneficial to performance [2]. In this sense, living systems are essentially stressed at all times, but the perceived effects and changes in biochemical responses are most apparent when the impacts are substantial. Further, the difference between a positive and negative response to stress may be exposure dependent. For instance, on a cellular level, a short-term glucocorticoid (cortisol) treatment resulted in improved mitochondrial function, whereas a long-term chronic exposure stimulated cell death [3]. It is then necessary to be aware of the variation in stress response of the same stimulant simply due to the manner the system is subjected to the stressor. 


\subsubsection{Contributors of stress and the impact on health}

Beyond describing stress in terms of positive and negative outcomes, it is also important to account for all potential factors contributing to overall stress in order to appropriately evaluate the response. Stressors can be generally classified by internal or external sources: internal stressors originate from the subject (e.g. overall health/nutrition, moods, thoughts, emotional state, personal goals and expectations) and external stressors encompass environmental stimulants that are imposed on the subject (e.g. physical trauma, chemical exposures, social relationships, major life changes, unexpected surprises). Additionally, the degree, frequency, and duration of the demand are all important aspects to consider for proper appraisal of stress. It is believed that the collective effects of prolonged or excessive contact to certain stressors can have an adverse impact on health. Some stressors, such as toxins or physical injuries, produce more obvious and immediate harm to one's wellbeing, but chronic distress can also lead to the development of physiological and psychiatric diseases [4]. These aftermaths can range from increased susceptibility to colds [5] or to depression [6]. There is still an ongoing effort to elucidate the link between stress and the onset of ailments [7-8], as the total contributors of stress are case-specific, sometimes difficult to control, and often challenging to replicate.

\subsubsection{Assessment of stress}

To evaluate the extent of stress that a living system can experience, it is important to select the appropriate array of measurements, which may depend on the stressors and the living system. For instance, to assess the effect of a chemical exposure, a number of different quantitative responses can be monitored (viability, metabolic activity, respiratory rates, death/necrosis, signaling, immune biomarker alterations, genetic modifications). When applicable (i.e. 
investigating human subjects that are able to provide feedback), alternative assessments that target a more qualitative description of the stress include standardized surveys and questionnaires based on the Perceived Stress Scale [9] or the Ardell Wellness Model [10]. These self-evaluating stress assessments are informative in cases when there is ambiguity in experienced stress among the tested subjects and are often used in conjunction with other biological measurements, such as heart rate variability [11] or hormones [12].

While yielding additional information, the relationship between biochemical measurements and self-reported stress is still a challenge to decipher for several reasons. First, there are biases in reported and observed stress that are difficult to control or prevent. An occurrence known as socially desirable responding (SDR) describes the propensity for people to put forth a more positive appearance, influencing the information that is provided [13]. In other cases where stress is observed by the investigator, such as assessing a physical injury, protocols for accurate, consistent, and subjective evaluation have yet to be developed [14]. In addition, the ambiguous and multiple roles of certain measured responses question the reliability of these "stress biomarkers". The contribution of cortisol as a stress hormone is a well-known example of this uncertainty due to its diverse roles in the regulation of metabolic processes (blood glucose levels, carbohydrate, protein, and lipid metabolism), inflammatory response, memory, as well as operation of the endocrine system [15]. Added complications, such as the time of the day [16], diet [17], and varying time scales of biological responses [18], can easily impact how the data is perceived and create obstacles that prevent a complete understanding of the stress response. Consequently, it is beneficial to collect and analyze both biochemical and survey data in 
complementary fashion, but it may also be advantageous to define a link between the two datasets [19].

\subsection{The biological response to stress}

In an effort to better comprehend and describe the stress response from a biological/biochemical perspective, it is imperative to first understand fundamental processes that regulate the basic necessities of a living system. Being aware of these baseline responses not only offers investigators with potential targets to monitor, but also provides a reference point as a comparison to determine if significant changes have actually occurred. For instance, to investigate the consequences of a chemical stressor with possible carcinogenic effects, it would be appropriate to monitor cellular alterations that regulate the balance between cell growth and death over an extended period of time (currently 2 year standard [20]). These cellular alterations could include genetic mutations, protein expression, modification, and signaling, or cell receptor impairments. Alternatively, to assess the effects of more conceptual stressors, such as workplace or social stress, exploring the involvement of individual immune or nervous system responses may be informative.

\subsubsection{The endocrine response}

Endocrines are also important components of the stress response and are managed by two particular systems, the hypothalamic-pituitary-adrenocortical (HPA) axis and the sympathetic-adrenal-medullary (SAM) system. Disrupting the homeostatic function of these systems, typically in the form of prolonged or repeated activity, can contribute to the onset and exacerbation of diseases and disorders [21]. A brief overview of the mechanisms and roles of each system will be covered in this section. 


\subsubsection{The hypothalamic-pituitary-adrenal axis}

The hypothalamic-pituitary-adrenal (HPA) axis is an important framework to address in the response to stress. The purpose of the HPA axis is to provide a controlled stream of regulatory hormones that manages widespread functions related to fetal organ development, metabolism, immunity, and cardiovascular maintenance. It is also a well-known key regulator in the adaptive response to stress and involves the coordination of the paraventricular nucleus (PVN) of the hypothalamus, the anterior lobe of the pituitary gland, and the adrenal gland. In brief, neurons in the hypothalamus secrete and release two hormones: corticotropin-releasing factor (CRF) and arginine vasopressin (AVP). These hormones flow through vasculature linking the hypothalamus and the pituitary gland, where the production and circulation of adrenocorticotropic hormone (ACTH) is prompted. Finally, ACTH stimulates the synthesis and distribution of glucocorticoids from the adrenal gland. Strict regulation of CRF, AVP, ACTH, and glucocorticoid production is implemented through a negative feedback of glucocorticoids, primarily cortisol [15].

While the CRF/AVP $\rightarrow$ ACTH $\rightarrow$ glucocorticoid (e.g cortisol) initiation and production loop is a mechanism that is constantly operating even in non-stressful situations, the influence of a stressor (psychological, trauma, or inflammatory) can alter HPA axis function. Exposure to positive stressors result in an initial amplification of HPA axis hormone production, followed by a quick decline in concentration that indicates the termination of the stressful incident. However, if the stress is too demanding, lengthy, or reoccurring, this could lead to deleterious effects as a result of the prolonged and amplified presence of cortisol, as well as the potential desensitization of the feedback mechanism [22]. A number of different factors can activate the HPA response, including cytokines [23], neurotransmitters, and other peptide hormones [24]. Changes to any of 
these hormone levels in the HPA axis can have a wide-ranging downstream effect on processes like blood glucose regulation, blood vessel dilation, inflammation [15].

\subsubsection{The sympathetic-adrenal-medullary system}

The sympathetic-adrenal-medullary (SAM) system accesses the autonomic nervous system (ANS), which manages activities that do not require conscious effort (breathing, digestion, swallowing). A particular type of hormone, called catecholamines, are secreted in the adrenal gland and are best recognized to be responsible for the "fight or flight" response. Catecholamines, such as dopamine, norepinephrine, and epinephrine can rapidly prompt increased breathing rate or blood flow to muscles, essentially providing an immediate means to react to an acute stressor. However, if these mechanisms are repeatedly activated due to a chronic stressor, negative outcomes have been observed: a weakened or altered immune system is a prime example of how irregular SAM system function can lead to adverse effects as catecholamines have the capacity to impede or amplify the immune response [25].

\subsubsection{The immune response}

The immune system is an integrated network of cells, tissues, and organs that provides a defense mechanism against foreign threats. The immune response can take two different, but interrelated paths: the innate or adaptive route. The innate response generally delivers a nonspecific immediate solution and is the first line of defense. The innate mechanism is primarily responsible for the recruitment of immune cells to the affected site, where necessary measures are taken to effectively manage the issue. Various types of cells (neutrophils, macrophages, eosinophils, basophils, lymphocytes, monocytes, natural killer cells, and dendritic cells) contribute 
to the overall response [26] and have been observed to perform a number of services that function to protect and minimize damage by trapping and eliminating the threat via phagocytosis (engulfing foreign material) [27] and/or the initiation of the adaptive immune response [28].

Adaptive immunity utilizes a more selective approach and possesses the capacity to retain information relevant to the specific elicited response for future deployment. Classically, adaptive immune processes are based on an antigen-antibody arrangement or a cell mediated response. Briefly, when a specific type of white blood cell (B cells) with membrane bound antibodies encounters an antigen (toxins, bacteria, foreign proteins) that binds to an antibody, it induces the production of more antibodies that then circulate throughout the bloodstream. These antibodies can detect and directly bind to the antigen to prevent them from binding to host cells and causing adverse outcomes [28]. Similarly, there are also T cells that can respond to antigens, but only if the antigen is already bound to receptors on antigen-presenting cells, such as macrophages. Depending on the type of receptor that the T cell expresses, foreign threats can be managed by the initiation of apoptosis in infected or non-viable cells via release of perforins and granzymes [29], further B cell activation, or prompting other immune responses [28].

\subsubsection{Relating immune response and stress}

While the relationship between the immune system and stress is a complex concept to unravel, considerable research has shown two perspectives of the relationship: how the immune system responds to stress and how stress impacts the immune system. The roles of immune mechanisms in mediating the effects of stress have been substantially explored to gain insight into how particular immune responses can be targeted to alleviate damage imposed by the stressor. For 
example, innate immune responses of traumatic injuries have been characterized to offer new treatment strategies or enhance existing therapeutic procedures [30, 31]. Furthermore, the immunotoxicity of a wide range of exposures, including endocrine disrupting chemicals [32] and nanomaterials [33] have also been examined to propose potential preventative measures that minimize adverse toxic outcomes.

Considering the immense contribution of the immune system in attending to a variety stressors, investigating the repercussions of too much stress imposed on immune function has additionally been a prominent focus of stress related research. Short-term stress is believed to provide an immuno-protective effect, while chronic stress has been associated with immunosuppressive outcomes [34]. It has been hypothesized that acute stress initially increases the production of immune cells, followed by a redistribution of the immune cells throughout the body. This process essentially supplies cells, tissues, and organs with a reserve of defenders in preparation for future attacks [35]. Acute stress is also known to enhance the memory capabilities of B cells, which is the essence of how vaccinations work. In cases of chronic stress, indications of a weakened immune system have been observed, such as diminished antibody response [36] and an overall decrease in immune cell production, function, and responsiveness [37]. When the "allostatic load" or the "wear and tear" a living system undergoes in response to a chronic stressor is too high, dysregulation or suppression of the immune system is a probable outcome [34].

\subsubsection{The cytokine response}

Another widely studied aspect of the biochemical stress response are cytokines - small peptides, proteins, and glycoproteins that activate a diverse spectrum of functions by binding to 
the surface receptors of a cell. Cytokines are known to be important signaling contributors of the immune response, as well as many other biological processes including, but not limited to, regulating and maintaining homeostatic conditions [38], embryonic development, inflammation, disease pathogenesis, cell proliferation, and cognitive function [39]. There are several features of cytokines that may contribute to its broad range of roles:

- They are pleiotropic - one cytokine can act on different cells and produce different effects [39].

- They can be redundant - different cytokines can trigger the same effects [40].

- They can be synergistic or antagonistic - combinations of cytokines can initiate or inhibit certain functions [41].

- They can behave as autocrines, paracrines, or endocrines - act on the same cell, on a cell nearby, or on a remote cell reached through circulation, respectively [40].

- They are produced in many different types of cells and can stimulate the expression of other cytokines and receptors - production and secretion can occur in immune (macrophages, B cells, T cells) [39], as well as endothelial and fibroblast cells [42].

As of 2008, several hundred cytokines and genes have been identified [43], with expectations to continue expanding on what is currently known. To summarize the features of the cytokine superfamily, it is common to organize the groups based on function, properties, or structure. Five cytokine classes will be briefly covered in this chapter: interleukins, interferons, tumor necrosis factors, chemokines, and colony-stimulating factors. 
Interleukins broadly encompass all secreted proteins that are primarily involved in the communication among leukocytes (white blood cells of the immune system). Even within this one class, there are dozens of functions that interleukins have been observed to exhibit. Akdis et al. compiled a list of more than 40 different interleukins along with their sources, target cells, major functions, and associated diseases [44], and demonstrated how complex and interrelated these interleukins can be. Many interleukins were reported to possess multiple functions, such as the induction of proinflammatory responses, cell proliferation, recruitment, and differentiation, apoptosis, and tumor suppression - to name a few.

Interferons (IFNs) are a group of cytokines involved in several diverse functions associated with anti-proliferation, pro-apoptosis, and cytotoxicity that have given this group potential to be used for clinical applications, such cancer treatments [45]. IFNs were first discovered to have a purpose in defending against viruses, but it appears they have a bigger role in regulating the immune response. Currently, IFNs are divided into two categories: Type I and Type II. Type I species, such as IFN- $\alpha$, IFN- $\beta$, or IFN- $\omega$, are induced by viral infections, while Type II (only consisting of IFN- $\gamma$ ) are stimulated by antigens or mitogens [46]. IFNs have also been strongly linked to the initiation of the JAK-STAT signaling pathway, which has subsequent downstream effects on various transcriptional activities [47].

Tumor necrosis factors (TNFs) have appropriately been described as multifunctional cytokines. As the name suggests, this family was initially believed to responsible for tumor cell apoptosis and cachexia, but many studies have provided evidence for a greater significance in mediating a number of different processes. In various cases, TNFs have exhibited anticancer and 
cytotoxic involvement, as well as signs of tumor promotion, angiogenesis, and invasion [48]. In addition, these cytokines have demonstrated substantial roles in the immuno-protective and proinflammatory response [49]. The widespread and sometimes contrasting nature of the TNF response not only illustrates the complexity of these regulatory processes, but also shows its flexibility that allows the biological system to be as efficient as possible.

Chemokines are believed to possess a more straightforward function, compared to the other previously described cytokines. They principally serve as chemotactic cytokines, directing the recruitment and movement of cells. Binding to the G-protein-coupled receptors on the target cell initiates intracellular signaling that regulate structural changes, such as membrane ruffling, pseudopod formation, and increased adhesive interactions, which drives the migration of cells towards a direction where they are needed [50]. Chemokines have been observed to act on many immune and non-immune cells (T cells, monocytes, neutrophils, NK cells, dendritic cells, fibroblasts, endothelial cells) [51], resulting in great interest in exploring chemokine roles in disease development and inflammatory processes [52].

Colony-stimulating factors (CSFS) are essential for the development, maturation, and differentiation of all bone marrow derived cells. Different forms of CSFs have been identified and named based on the type of cell that responds when stimulated. Three well established CSFs include granulocyte/macrophage colony-stimulating factor (GM-CSF), macrophage colonystimulating factor (M-CSF), and granulocyte colony-stimulating factor (G-CSF) [53]. Despite some differences in activation, sources, targets, and effects among the various CSFs, their overall efforts are concentrated to promote the survivability of cells. Consequently, these cytokines have 
been linked to various immune and inflammatory responses, progression of diseases, and have additionally been investigated as targets for therapeutic and clinical applications [54].

Another common system to define cytokines is based on their pro or anti-inflammatory effects. Inflammation in tissues can be characterized by redness, heat, pain, and swelling that can lead to dysfunction of organs, as well as the activation of the immune response on the cellular level [55]. Cytokines have been observed to both promote and reduce inflammation. Well established proinflammatory cytokines include IL-1, TNF, IL-12, IL-18, and GM-CSF and anti-inflammatory cytokines include IL-4, IL-10, IL-13, IFN- $\alpha$, and transforming growth factor [56]. Some cytokines, like IL-6, are known to exhibit both properties [57], suggesting that the notion of labeling cytokines as having either a pro or anti-inflammatory effect limits the way cytokine roles are perceived.

\subsubsection{Cytokine measurement}

Monitoring cytokines can be an informative approach to acquire a comprehensive depiction of the efforts taken in response to perturbations against a living system. There are multiple available methods to measure cytokine response that describe different aspects of cytokine activity. One option is to investigate the effects of the cytokine of interest (e.g. the initiation of cell proliferation or chemotaxis can be analyzed). Alternatively, immunoassays, such as enzyme linked immunosorbent assays can measure specific cytokine concentrations based on antibody-specific interactions with targets. Other methods include receptor binding [58] or gene expression [59] analysis that can provide additional information about cytokine activity. However, investigators must be cautious as there are many factors to consider. Besides selecting the appropriate cytokines that are most relevant to the context from the large collection of all known targets, it is important 
to be aware of elements that may interfere with assays or influence the results. Uncertainty may be attributed to:

- Degradation of cytokines (during storage, freeze/thaw cycles, or naturally occurring) [60]

- Additional cellular cytokine production and release post-sample collection [60]

- Interactions/binding of cytokines to membrane receptors, proteins, autoantibodies [60]

- Timing of cytokine existence (short half-life, circadian rhythms) [60]

- Different forms of cytokines (precursors, glycosylated products, polymeric structures) [60]

- Limit of detection (LOD): Levels of circulating cytokines in blood are generally below the LOD with commercially available assays [61].

As cytokines are multifunctional, involved in many important regulatory and defense mechanisms, and have been linked to many diseases and disorders, there is promising potential in observing cytokine biomarkers for both research and clinical applications. Much progress has been made in developing methods for cytokine measurement, but more work is needed to address all of the uncertainties and ranges of cytokine behavior. In this regard, there are limitations to how cytokine response can be interpreted and for now, the best way to alleviate any reservations is to account for and include all relevant experimental controls.

\subsubsection{Cytokines and Stress}

The roles of cytokines in the context of stress is an extremely vast field of research. Whether it is the response resulting from a physical stressor (exercise [62] or injury [63]), hazardous exposure [64], or diseases [65] and psychological disorders [66-67] exacerbated by 
stress, cytokine response is recognized as a powerful tool to assess the impact of stressors and to elucidate disease pathogenesis, as well as potential treatments. There is considerable interest in using cytokine biomarkers to characterize different forms of stress [68], but there are also challenges due to significant variations in cytokine levels among individuals, multiple functions and triggers of cytokine activation, as well as concentration discrepancies in different biological matrix. Consequently, more studies need to be conducted with matching stressors and sample matrices to establish accurate cytokine profiles.

\subsubsection{Intracellular protein signal transduction}

Exploring systemic responses, such as the immune or endocrine system, provides a big picture of the protective countermeasures implemented to promote a healthy state. However, it is critical to note that systemic signaling, initiation, and activation are followed or mediated by a cascade of intracellular events. Intracellular protein expression and signaling are essential components of sustaining homeostatic conditions and carrying out defensive mechanisms. For instance, phagocytosis (immune cells engulfing foreign bodies), is driven by cell movement, engulfment, and sterilization that are all dependent on protein signaling and structural support [69].

The mitogen activated protein kinase (MAPK) family is currently the best characterized protein signaling pathway that is known to be involved in cellular proliferation, differentiation, development and even cell death. These proteins act as messengers and deliver signals that are received at the surface receptors to other intracellular mediators that release subsequent secondary signals or to the nucleus, where gene transcription takes place. Within this family, the ERK1/2, JNK, and p38 pathways are frequently investigated, which has resulted in an abundant and 
comprehensive literature base regarding their activity. Certain cascades, such as Ras-Raf-MEKERK or JAK-STAT, are recognized as conserved pathways and many downstream targets or substrates have been identified as well [70], but crosstalk among distinct pathways is also vital for efficient communication and operation [71]. When investigating protein expression and signaling, it is beneficial to select groups of proteins that are known to work together as a network in order to piece together an image of cellular efforts.

There are many ways signals can be transduced. Protein phosphorylation is one of the principal forms of transmitting signals and is often studied to acquire a better understanding of cellular communication and function [72]. The process of phosphorylation is mediated by kinases and requires a source of energy in the form of adenosine triphosphate (ATP). Conversely, proteins can also undergo de-phosphorylation, facilitated by phosphatases. Measuring both protein expression and phosphorylated protein levels can reveal substantial information about the overall response. Amplification of certain proteins may indicate the activation of cellular processes that are associated with those proteins. Phosphorylation signaling can further elucidate which pathways are being activated that contribute to the outcome. It must be noted that increased or decreased phosphorylation does not necessarily correspond to activation or deactivation of the protein, respectively. In fact, it is well known that a single protein can have multiple phosphorylation sites and site-specific phosphorylation can dictate function [73]. Moreover, studies have shown that all types of proteins can ultimately be inactivated by phosphorylation [74-76]. 


\subsection{Biological samples}

When measuring any stress response in living subjects, there are different options regarding the biological matrix that can be sampled. In most cases, the selected matrix will depend on the affected tissue, measurement of interest, or even the accessibility of sample collection. For instance, to investigate the biochemical response of physical stressors (e.g. physical trauma), the impact is localized and a resection of the damaged tissue would be an appropriate sample to collect. In contrast, if the objective is to explore a more systemic response with a broad spread of biochemical communication (e.g. HPA axis), then working with blood, saliva, or even cerebral spinal fluid from a human subject would be suitable. Great consideration must be taken in selecting a sample matrix that will best capture the desired response. In addition, there is always great interest in collecting samples that are minimally invasive, easy to sample, and cause low risks to the subject and the researcher. As a result, biological matrices, such as saliva, blood, sweat, hair, urine, and tears, are all favorable options. The work covered in this dissertation focused on three types of biological matrices: resected ex vivo muscle tissue, saliva, and blood.

\subsubsection{Ex vivo tissue}

Ex vivo assessments can be advantageous for obtaining a spatiotemporal snapshot of the response. It is assumed that basal biological activity and any changes that transpired due to a stressor is preserved in properly extracted, stored, and handled tissue. It may also be necessary to phenotype or characterize the different components of the isolated tissue. For instance, if the immune response is being investigated, isolating immune cells allows for a focused investigation and removes noise produced by other cell types. Awareness of the potential variability in sampling is also critical. It is challenging to have complete control over the consistency in tissue samples 
that can be influenced by factors as simple as the size of the sample. Differences in size may be an issue if there is a spatial gradient of the response that yields an increased or reduced apparent effect, compared to the true response.

\subsubsection{Saliva}

Saliva is viewed as one of the more convenient diagnostic mediums that has the capacity to detect both local and systemic irregularities. The identification of salivary biomarkers that are indicative of infections, autoimmune disease, carcinoma, endocrine dysfunction, and stress is being pursued with great potential for success [77]. It is important that investigators are aware of some of the major factors in saliva sample collection that can affect the outcome. First, there are many major and minor salivary glands that produce and secrete saliva. The major glands include the parotid, submandibular, and sublingual, while minor glands are spread out in locations such as the tongue, tonsils, palate, and cheeks. Whole saliva that is produced from multiple gland sources can be collected, or alternatively saliva can be extracted from individual glands to learn more about the association between diseases and specific saliva sources [78]. Other potential complications can include poor oral health (any cuts or sores, inflammation, gingivitis), circadian rhythms, oral stimulants and contamination (food, water, gum) [79], and dehydration [80].

Stimulation of saliva can be triggered by physical and mechanical factors, such as chewing or talking, but the autonomic nervous system also plays a substantial role. Parasympathetic and sympathetic nerves innervate salivary glands and transmit signals that regulate fluid and protein secretion. Acetylcholine and noradrenaline are neurotransmitters produced by parasympathetic and sympathetic nerves, respectively, and are chiefly responsible for activating salivary cell 
secretion. A number of different proteins (amylases, carbonic anhydrase, histatins, mucins, proline-rich proteins) have been detected in saliva and are either secreted continuously or activated by the nervous system [81]. Additionally, salivary glands feature permeable capillaries that permit the exchange of molecules found circulating in the blood and in salivary cells, supporting the idea that systemic responses and biomarkers that are not localized in the oral cavity can be detected in saliva [82].

\subsubsection{Blood and plasma}

Blood tests are the most common form of monitoring physiological conditions. Whether it is a measurement of glucose concentration, white blood cell counts, or cholesterol levels, blood is a dynamic and powerful diagnostic medium. Blood is comprised of two main components: the plasma and formed elements. The plasma is mostly water (>90\%), followed by proteins (albumins, globulins, fibrinogens, regulatory proteins), and other solutes (electrolytes, nutrients, waste). The formed elements include red blood cells (> 99\%), white blood cells, and platelets. Blood performs a variety of tasks, the most obvious being transportation of gases, nutrients, wastes, signaling hormones and proteins. As a result, the contents detected in blood can provide valuable information about systemic efforts taken in response to stressors, diseases, and disorders.

\subsection{Bioinformatics: analyzing complex biological datasets}

There are many biological, physiological, biochemical, systemic, and localized measurements that can be taken to investigate the stress response, which can potentially lead to a large and complex dataset. In addition to selecting logical responses to monitor, an equally important aspect of research is data analysis and fortunately, statisticians and biostatisticians have 
demonstrated many different approaches that can be employed to piece together the data and extract pertinent information. The complexity of datasets may be attributed to the considerable number of biological components and processes involved, as well as the potential coordination and crosstalk that occurs, or even the design of the experiment (number of doses, time points, sample types). While identifying significant differences or associations among conditions using traditional statistic methods, such as analysis of variance (ANOVA), t-test, or correlation analysis is an acceptable approach, a more sophisticated statistical treatment may be needed to properly assess this complex data.

\subsubsection{Network analysis}

Biological processes typically transpire as pathways, where a ligand triggers an action by binding to a receptor and then passing on the signal through cascades until the desired response is achieved. Crosstalk or interactions among pathways also occur, resulting in a network of relevant pathways. Networks are portrayed as a structure of nodes and edges; nodes represent any measured response (e.g. proteins, cytokines) and edges represent the interaction between the nodes. Common networks include metabolic networks, kinase-substrate networks, gene regulatory networks, protein-protein interaction networks, and drug interaction networks [83]. Beyond just the construction of these networks, the associated properties or parameters are often examined to better understand the connections within the network and the contribution of certain nodes. Several network centrality parameters have been identified [84]:

- Degree: number of nodes directly connected to a given node

- Diameter: the distance between the two most distant nodes in the network 
- Average distance: sum of all the shortest paths between each pair of node divided by the number of node pairs

- Eccentricity: the inverse of the distance of the longest direct path between a given node and all other nodes in the network.

- Closeness: the inverse of the sum of all direct paths between a given node and all other nodes in the network

- Radiality: the sum of the shortest paths between a given node and all other nodes, normalized to the network diameter, divided by the number of nodes - 1

- Centroid value: a systematic counting of nodes that are closer (shorter distance) to one node versus a second node

- Stress: a count of the shortest paths in the network that pass through a given node

- Betweenness: the number of shortest paths that pass through a specific node, relative to the total number of shortest paths

\subsubsection{Alternative analysis approaches}

Other statistical methods, including principal component analysis (PCA), discriminant analysis, and clustering analysis are commonly employed to simplify dynamic datasets. PCA works best with datasets that contain a large number of variables by computing new descriptive elements (principal components) based on the variability within the dataset. By generating these principal components, the dimensions and the complexity of the dataset are significantly reduced. Discriminant analysis is frequently used as a predictive method that is capable of classifying observations into pre-established groups based on the mathematical fitting of measured responses, while cluster analysis groups responses that are similar depending on the magnitude of the 
measured response. All of the mentioned analyses have proven to be reliable approaches in properly managing large and complex datasets. Determining which method to use simply depends on the desired outcomes, as well as the format of the data (e.g classification groups must be provided for discriminant analysis).

\subsection{References}

[1] Selye H. The Stress of Life, McGraw-Hill, New York, NY. 1956.

[2] Le Fevre M, Matheny J, Kolt GS. Eustress, distress, and interpretation in occupational stress. J Manag Psychol. 2003;18(7):726-744.

[3] Du J, Wang Y, Hunter R, Wei Y, Blumenthal R, Falke C, et al. Dynamic regulation of mitochondrial function by glucocorticoids. Proc Natl Acad Sci U S A. 2009;106(9):3543-8.

[4] Cohen, S., Kessler, R., \& Gordon, L. Strategies for measuring stress in studies of psychiatric and physical disorders. New York, NY: Oxford University Press. 1995.

[5] Cohen S, Frank E, Doyle WJ, Skoner DP, Rabin BS, Gwaltney JM, Jr. Types of stressors that increase susceptibility to the common cold in healthy adults. Health Psychol. 1998;17(3):214-23.

[6] Hammen C. Stress and depression. Annu Rev Clin Psychol. 2005;1:293-319.

[7] Edmondson D, von Kanel R. Post-traumatic stress disorder and cardiovascular disease. Lancet Psychiatry. 2017;4(4):320-9.

[8] Mortensen J, Dich N, Lange T, Alexanderson K, Goldberg M, Head J, et al. Job strain and informal caregiving as predictors of long-term sickness absence: A longitudinal multi-cohort study. Scand J Work Environ Health. 2017;43(1):5-14. 
[9] Cohen S, Kamarck T, Mermelstein R. A global measure of perceived stress. J Health Soc Behav. 1983;24(4):385-96.

[10] Ardell DB. A wellness model for national health insurance. J Health Hum Resour Adm. $1983 ; 5(3): 321-33$.

[11] Satyapriya M, Nagendra HR, Nagarathna R, Padmalatha V. Effect of integrated yoga on stress and heart rate variability in pregnant women. Int J Gynaecol Obstet. 2009;104(3):218-22.

[12] Corbett BA, Schupp CW, Levine S, Mendoza S. Comparing cortisol, stress, and sensory sensitivity in children with autism. Autism Res. 2009;2(1):39-49.

[13] Van de Mortel TF. Faking it: social desirability response bias in self-report research. Aust J Adv Nurs. 2008;40:40-48.

[14] Grey JE, Enoch S, Harding KG. Wound assessment. BMJ. 2006; 332(7536):285-8.

[15] Stephens MA, Wand G. Stress and the HPA axis: role of glucocorticoids in alcohol dependence. Alcohol Res. 2012;34(4):468-83.

[16] Fernandes AL, Lopes-Silva JP, Bertuzzi R, Casarini DE, Arita DY, Bishop DJ, et al. Effect of time of day on performance, hormonal and metabolic response during a 1000-M cycling time trial. PLoS One. 2014;9(10):e109954.

[17] Kitaysky AS, Kitaiskaia EV, Wingfield JC, Piatt JF. Dietary restriction causes chronic elevation of corticosterone and enhances stress response in red-legged kittiwake chicks. J Comp Physiol B. 2001;171(8):701-9.

[18] Oken BS, Chamine I, Wakeland W. A systems approach to stress, stressors and resilience in humans. Behav Brain Res. 2015;282:144-54. 
[19] Han AA, Vrana JA, Mitchell C, Boyd JW. Identification of Biomarkers Related to Perceived Stress Scale-10 and the Evaluation of the Survey Components. J Med Genomics Biomark. 2015;2(003).

[20] Food and Drug Administration, Guidance for Industry: Carcinogenicity Study Protocol Submissions, FDA, Rockville, MD, 2002.

[21] Cohen S, Janicki-Deverts D, Miller GE. Psychological stress and disease. Jama. 2007;298(14):1685-7.

[22] Smith SM, Vale WW. The role of the hypothalamic-pituitary-adrenal axis in neuroendocrine responses to stress. Dialogues Clin Neurosci. 2006;8(4):383-95.

[23] Turnbull AV, Rivier CL. Regulation of the hypothalamic-pituitary-adrenal axis by cytokines: actions and mechanisms of action. Physiol Rev. 1999;79(1):1-71.

[24] Ulrich-Lai YM, Herman JP. Neural Regulation of Endocrine and Autonomic Stress Responses. Nat Rev Neurosci. 2009;10(6):397-409.

[25] Madden KS. Catecholamines, sympathetic innervation, and immunity. Brain Behav Immun. 2003;17 Suppl 1:S5-10.

[26] Warrington R, Watson W, Kim HL, Antonetti FR. An introduction to immunology and immunopathology. Allergy Asthma Clin Immunol. 2011;7 Suppl 1:S1.

[27] Parkin J, Cohen B. An overview of the immune system. Lancet. 2001;357(9270):1777-89.

[28] Alberts B, Johnson A, Lewis J, et al. Molecular Biology of the Cell. 4th edition. New York: Garland Science; 2002. Chapter 24, The Adaptive Immune System. 
[29] Voskoboinik I, Whisstock JC, Trapani JA. Perforin and granzymes: function, dysfunction and human pathology. Nat Rev Immunol. 2015;15(6):388-400.

[30] Hietbrink F, Koenderman L, Rijkers G, Leenen L. Trauma: the role of the innate immune system. World J Emerg Surg. 2006;1:15.

[31] Osuka A, Ogura H, Ueyama M, Shimazu T, Lederer JA. Immune response to traumatic injury: harmony and discordance of immune system homeostasis. Acute Med. Surg. 2014;1(2):63-9.

[32] Xu H, Yang M, Qiu W, Pan C, Wu M. The impact of endocrine-disrupting chemicals on oxidative stress and innate immune response in zebrafish embryos. Environ Toxicol Chem. 2013;32(8):1793-9.

[33] Elsabahy M, Wooley KL. Cytokines as biomarkers of nanoparticle immunotoxicity. Chem Soc Rev. 2013;42(12):5552-76.

[34] Dhabhar FS. Effects of stress on immune function: the good, the bad, and the beautiful. Immunol Res. 2014;58(2-3):193-210.

[35] Dhabhar FS, Miller AH, McEwen BS, Spencer RL. Effects of stress on immune cell distribution. Dynamics and hormonal mechanisms. J Immunol. 1995;154(10):5511-27.

[36] Cohen S, Miller GE, Rabin BS. Psychological stress and antibody response to immunization: a critical review of the human literature. Psychosom Med. 2001;63(1):7-18.

[37] Dragos D, Tanasescu MD. The effect of stress on the defense systems. J Med Life. 2010;3(1):10-8.

[38] Boyman O, Purton JF, Surh CD, Sprent J. Cytokines and T-cell homeostasis. Curr Opin Immunol. 2007;19(3):320-6. 
[39] Dinarello CA. Historical Review of Cytokines1. Eur J Immunol. 2007;37(Suppl 1):S34-45.

[40] Cohen MC, Cohen S. Cytokine function: a study in biologic diversity. Am J Clin Pathol. 1996;105(5):589-98.

[41] Turrin NP, Plata-Salaman CR. Cytokine-cytokine interactions and the brain. Brain Res Bull. 2000;51(1):3-9.

[42] Savinko T, Matikainen S, Saarialho-Kere U, Lehto M, Wang G, Lehtimaki S, et al. IL-33 and ST2 in atopic dermatitis: expression profiles and modulation by triggering factors. J Invest Dermatol. 2012;132(5):1392-400.

[43] Khan MM. Role of Cytokines, Immunopharmacology; Springer: New York, NY, USA, 2008:43-59.

[44] Akdis M, Burgler S, Crameri R, Eiwegger T, Fujita H, Gomez E, et al. Interleukins, from 1 to 37, and interferon-gamma: receptors, functions, and roles in diseases. J Allergy Clin Immunol. 2011;127(3):701-21.e1-70.

[45] Pestka S, Krause CD, Walter MR. Interferons, interferon-like cytokines, and their receptors. Immunol Rev. 2004;202:8-32.

[46] Goodbourn S, Didcock L, Randall RE. Interferons: cell signalling, immune modulation, antiviral response and virus countermeasures. J Gen Virol. 2000;81(Pt 10):2341-64.

[47] Samuel CE. Antiviral actions of interferons. Clin Microbiol Rev. 2001;14(4):778-809, table of contents.

[48] Wang X, Lin Y. Tumor necrosis factor and cancer, buddies or foes? Acta Pharmacol Sin. 2008;29(11):1275-88. 
[49] Pfeffer K. Biological functions of tumor necrosis factor cytokines and their receptors. Cytokine Growth Factor Rev. 2003;14(3-4):185-91.

[50] Luster AD. Chemokines--chemotactic cytokines that mediate inflammation. N Engl J Med. $1998 ; 338(7): 436-45$.

[51] Rollins BJ. Chemokines. Blood. 1997;90(3):909-28.

[52] Sallusto F, Baggiolini M. Chemokines and leukocyte traffic. Nat Immunol. 2008;9(9):949-52.

[53] Wakefield PE, James WD, Samlaska CP, Meltzer MS. Colony-stimulating factors. J Am Acad Dermatol. 1990;23(5 Pt 1):903-12.

[54] Hamilton JA. Colony-stimulating factors in inflammation and autoimmunity. Nat Rev Immunol. 2008;8(7):533-44.

[55] Libby P. Inflammatory mechanisms: the molecular basis of inflammation and disease. Nutr Rev. 2007;65(12 Pt 2):S140-6.

[56] Cavaillon JM. Pro- versus anti-inflammatory cytokines: myth or reality. Cell Mol Biol. 2001;47(4):695-702.

[57] Scheller J, Chalaris A, Schmidt-Arras D, Rose-John S. The pro- and anti-inflammatory properties of the cytokine interleukin-6. Biochim Biophys Acta. 2011;1813(5):878-88.

[58] Thorpe R, Wadhwa M, Bird CR, Mire-Sluis AR. Detection and measurement of cytokines. Blood Rev. 1992;6(3):133-48.

[59] Di Nicola M, Cattaneo A, Hepgul N, Di Forti M, Aitchison KJ, Janiri L, et al. Serum and gene expression profile of cytokines in first-episode psychosis. Brain Behav Immun. 2013;31:90-5. 
[60] Bienvenu J, Monneret G, Fabien N, Revillard JP. The clinical usefulness of the measurement of cytokines. Clin Chem Lab Med. 2000;38(4):267-85.

[61] Aziz N. Measurement of Circulating Cytokines and Immune-Activation Markers by Multiplex Technology in the Clinical Setting: What Are We Really Measuring? For Immunopathol Dis Therap. 2015;6(1-2):19-22.

[62] Dimitrov S, Shaikh F, Pruitt C, Green M, Wilson K, Beg N, et al. Differential TNF production by monocyte subsets under physical stress: blunted mobilization of proinflammatory monocytes in prehypertensive individuals. Brain Behav Immun. 2013;27(1):101-8.

[63] Haslauer CM, Proffen BL, Johnson VM, Hill A, Murray MM. Gene expression of catabolic inflammatory cytokines peak before anabolic inflammatory cytokines after ACL injury in a preclinical model. J Inflamm (Lond). 112014.

[64] Raghnaill MN, Bramini M, Ye D, Couraud PO, Romero IA, Weksler B, et al. Paracrine signalling of inflammatory cytokines from an in vitro blood brain barrier model upon exposure to polymeric nanoparticles. Analyst. 2014;139(5):923-30.

[65] Calcagni E, Elenkov I. Stress system activity, innate and T helper cytokines, and susceptibility to immune-related diseases. Ann NY Acad Sci. 2006;1069:62-76.

[66] Slavich GM, Irwin MR. From stress to inflammation and major depressive disorder: a social signal transduction theory of depression. Psychol Bull. 2014;140(3):774-815.

[67] Goines PE, Ashwood P. Cytokine dysregulation in autism spectrum disorders (ASD): possible role of the environment. Neurotoxicol Teratol. 2013;36:67-81. 
[68] Monastero RN, Pentyala S. Cytokines as Biomarkers and Their Respective Clinical Cutoff Levels. Int J Inflam. 2017;2017:4309485.

[69] Aderem A, Underhill DM. Mechanisms of phagocytosis in macrophages. Annu Rev Immunol. 1999;17:593-623.

[70] Zhang W, Liu HT. MAPK signal pathways in the regulation of cell proliferation in mammalian cells. Cell Res. 2002;12(1):9-18.

[71] Fey D, Croucher DR, Kolch W, Kholodenko BN. Crosstalk and signaling switches in mitogen-activated protein kinase cascades. Front Physiol. 2012;3:355.

[72] Paul A, Wilson S, Belham CM, Robinson CJ, Scott PH, Gould GW, et al. Stress-activated protein kinases: activation, regulation and function. Cell Signal. 1997;9(6):403-10.

[73] Lundby A, Secher A, Lage K, Nordsborg NB, Dmytriyev A, Lundby C, et al. Quantitative maps of protein phosphorylation sites across 14 different rat organs and tissues. Nat Commun. 2012;3:876.

[74] Sutherland C, Cohen P. The alpha-isoform of glycogen synthase kinase-3 from rabbit skeletal muscle is inactivated by p70 S6 kinase or MAP kinase-activated protein kinase-1 in vitro. FEBS Lett. 1994;338(1):37-42.

[75] Lee SN, Hwang JR, Lindberg I. Neuroendocrine protein 7B2 can be inactivated by phosphorylation within the secretory pathway. J Biol Chem. 2006;281(6):3312-20.

[76] Datta SR, Katsov A, Hu L, Petros A, Fesik SW, Yaffe MB, et al. 14-3-3 proteins and survival kinases cooperate to inactivate BAD by BH3 domain phosphorylation. Mol Cell. 2000;6(1):41-51.

[77] Malamud D. Saliva as a diagnostic fluid. Dent Clin North Am. 2011;55(1):159-78. 
[78] Ryu OH, Atkinson JC, Hoehn GT, Illei GG, Hart TC. Identification of parotid salivary biomarkers in Sjogren's syndrome by surface-enhanced laser desorption/ionization time-of-flight mass spectrometry and two-dimensional difference gel electrophoresis. Rheumatology (Oxford). 2006;45(9):1077-86.

[79] Martí-Álamo S, Mancheño-Franch A, Marzal-Gamarra C, Carlos-Fabuel L. Saliva as a diagnostic fluid. Literature review. J Clin Exp Dent. 42012. p. e237-43.

[80] Ship JA, Fischer DJ. The relationship between dehydration and parotid salivary gland function in young and older healthy adults. J Gerontol A Biol Sci Med Sci. 1997;52(5):M310-9.

[81] Ekström J, Khosravani N, Castagnola M, Messana I. Saliva and the control of its secretion. In Dysphagia 2011 (pp. 19-47). Springer Berlin Heidelberg.

[82] Yoshizawa JM, Schafer CA, Schafer JJ, Farrell JJ, Paster BJ, Wong DT. Salivary biomarkers: toward future clinical and diagnostic utilities. Clin Microbiol Rev. 2013;26(4):781-91.

[83] Ma'ayan A. Introduction to Network Analysis in Systems Biology. Sci Signal. 2011;4(190):tr5.

[84] Scardoni G, Laudanna C. Centralities based analysis of complex networks. In TECH Open Access Publisher; 2012. 


\section{Chapter 2}

Spatiotemporal phosphoprotein distribution and associated cytokine response of a traumatic injury 


\section{Spatiotemporal phosphoprotein distribution and associated cytokine response of a traumatic injury ${ }^{1}$}

Molecular mechanisms of wound healing have been extensively characterized, providing a better understanding of the processes involved in wound repair and offering advances in treatment methods. Both spatial and temporal investigations of injury biomarkers have helped to pinpoint significant time points and locations during the recovery process, which may be vital in managing the injury and making the appropriate diagnosis. This study addresses spatial and temporal differences of phosphoproteins found in skeletal muscle tissue following a traumatic femur fracture, which were further compared to co-localized cytokine responses. In particular, several proteins (Akt, ERK, c-Jun, CREB, JNK, MEK1, and p38) and post-translational phosphorylations (p-Akt, p-c-Jun, p-CREB, p-ERK1/2, p-MEK1, p-p38, p-GSK3a/b, p-HSP27, pp70S6K, and p-STAT3) associated with inflammation, new tissue formation, and remodeling were found to exhibit significant spatial and temporal differences in response to the traumatic injury. Quadratic discriminant analysis of all measured responses, including cytokine concentrations from previously published findings, was used to classify temporal and spatial observations at high predictive rates, further confirming that distinct spatiotemporal distributions for total protein, phosphorylation signaling, and cytokine (IL-1a, IL-1ß, IL2, IL6, TNF-a, and MIP-1a) responses exist. Finally, phosphoprotein measurements were found to be significantly correlated to cytokine concentrations, suggesting coordinated intracellular and extracellular activity during crucial periods of repair. This study represents a first attempt to monitor and assess integrated changes in extracellular and intracellular signaling in response to a traumatic injury in muscle tissues, which

\footnotetext{
1 Parts of this chapter have been published previously from Han AA, Currie HN, Loos MS, Vrana JA, Fabyanic EB, Prediger MS, et al. Spatiotemporal phosphoprotein distribution and associated cytokine response of a traumatic injury. Cytokine. 2016;79:12-22. Reproduced with permission from Elsevier.
} 
may provide a framework for future research to improve both our understanding of wounds and their treatment options.

\subsection{Introduction}

Wound healing in response to a traumatic injury is a highly complex process that is regulated by a myriad of coordinated biological processes and mechanisms. Conserved responses observed in wound repair have been classically characterized by three general stages: inflammation, new tissue formation, and remodeling [1]. While each stage serves a specific function and occurs at predictive time points, the healing process is described to transpire in continuous interdependent cellular and molecular steps that have not yet been fully elucidated [2]. Impairment of central steps, influenced by the severity and management of the wound, may delay repair or lead to additional detrimental risks, such as the development of non-viable tissue [3]. A heightened understanding of innate wound healing mechanisms offers opportunities to enhance medical treatments [4] and prevent further complications. Specifically, temporal and spatial changes of injury biomarkers are areas that have yet to be fully elucidated, but present prospective approaches to accelerate or aid in the recovery process. For example, progressive wound treatment strategies, such as tissue engineering, can be improved through the elucidation of spatial molecular patterns in injured tissue [5]. Additionally, standard techniques used to facilitate wound healing, such as surgical debridement [6] can be further refined through a better understanding of the spatiotemporal distribution of injury biomarkers. Determining the spatial aspect of repair may potentially aid surgeons to better determine which tissue to debride, while the temporal aspect of repair may help predict the outcome of injured tissue. 
Most molecular level tissue injury response studies have focused on the time course of responses following injury, but the spatial aspect of molecular injury responses can also provide valuable insight for the understanding and treatment of traumatic wounds [7]. Several studies have reported various mechanistic features involved in healing: temporal and tissue dependent distributions of protein expression [8], temporal response of growth factors [9] and chemokines [10], and temporal and spatial responses of cytokines [11]. However, there has not yet been an investigation evaluating spatial and temporal phosphoprotein responses following a traumatic fracture injury. Protein phosphorylation is a fundamental and vital process centrally involved in many cellular processes that are intimately involved in the response to traumatic injury, including a role in the regulation of cell (and ultimately tissue) survival [12]. For instance, phosphorylation activity is notably involved in initiating cellular signaling cascades that are vital to all three stages of wound healing [13-15]. Thus, activation or deactivation of phosphoproteins, as well as fluctuations in phosphorylation levels, may describe cell signaling activity in states of cellular distress or different phases of recovery [16]. Examining phosphorylation changes under injured conditions lends a depiction of the healing process from a signaling aspect that may further advance the understanding and treatment of wounds.

Protein phosphorylation is a primary means by which extracellular signals, such as cytokines, are integrated within target cells, allowing cells to respond in a regulated manner and carry out the wound healing process. Phosphorylation levels observed in various severities of injury, ranging from traumatic brain injury [17] to dermal chemical burns [18], have been shown to exhibit distinct temporal response patterns. Certain phosphoproteins hold an especially central role in cell growth and survival, such as those within the mitogen-activated protein kinase (MAPK) 
family. MAPK signaling is widely integrated in many processes responding to cellular distress. For instance, the involvement of increased MAPK signaling is becoming progressively prominent as a marker in many inflammatory diseases [19]. ERK1/2 [20], MEK [21], and JNK [22] are major components in the MAPK signaling pathway that have been described to significantly experience alterations during the repair process. Additionally, the coordination between MAPK pathway phosphorylation mediated signaling and other regulatory elements, such as growth factors, has been observed in cases of injury and wound healing [23].

In our previous research [11], we demonstrated a spatial cytokine distribution that exists following a Gustilo III-B [24] leg fracture in rats. As a continuation of that study, we have investigated the spatial and temporal intracellular phosphoprotein response in skeletal muscle tissue following traumatic injury. This study is the first to address measurable spatial and temporal differences of phosphoproteins found in muscle tissue following a traumatic injury severe enough to cause a bone fracture. Total levels of proteins in the MAPK family, along with an additional cohort of tightly associated downstream protein kinases, were measured in order to identify the differential distribution in response to the injury. Phosphorylation levels of proteins were also measured with an intention to further explore signaling activity during the recovery process. Spatial and temporal differences of all measured responses were statistically identified using ANOVA, and quadratic discriminant analysis was additionally used to classify temporal and spatial observations. Phosphoprotein responses measured in this study were also found to be related to previously published spatial and temporal cytokine responses [11]. Specifically, correlation analysis was conducted using total protein, phosphorylation, and previously published cytokine levels (measured from the same collected tissues) from Currie 2014, which for the first 
time allows a spatiotemporal examination of the coordinated relationship between cytokines and phosphoprotein (i.e. extracellular and intracellular) responses post traumatic injury. Overall, this research represents an alternative view of cellular response to a traumatic injury that offers an enhanced understanding of the underlying mechanisms that are involved in both initial response and repair.

\subsection{Materials and methods}

\subsubsection{Animals}

Adult male Sprague-Dawley rats were housed individually with a 12:12 light/dark cycle with ad libitum access to standard rat chow and water. Four time points were studied with 3 replicates each $(\mathrm{N}=3)$ for a total of 12 rats for the study. All procedures were performed under the guidelines approved by the West Virginia University Animal Care and Use Committee.

\subsubsection{Femur fracture}

Buprenorphine SR was pre-operatively administered subcutaneously as an analgesic providing $72 \mathrm{~h}$ pain relief. Rats were anesthetized intraperitoneally with Ketamine ( $80-90 \mathrm{mg} / \mathrm{kg}$ ) and Xylazine $(10-15 \mathrm{mg} / \mathrm{kg})$. This combination of analgesic and anesthetics has previously been identified as the best combination for avoiding significant modulation of cytokine responses in a rat model [25]. Analgesics and anesthetics were additionally injected into the scruff on the back of the rat's neck. After administration of anesthesia, all animals were subjected to a standardized femur fracture on one leg using a custom designed tool in which a weight is dropped in a consistent fashion onto the mid-shaft of the rat's thigh [26]. This tool delivers a calculated force of 104.80 
Newtons, generating a reproducible femur fracture and associated soft tissue injury. An incision was made to visualize the fracture to allow drilling of a hole into the proximal femur to allow a 0.045 in. Kirschner wire (K-wire) to be inserted down the intramedullary canal to fix the fracture. The incision was closed starting with the fascia, followed by a stainless steel suture on the skin. Rats were subcutaneously administered Yohimbine $(2 \mathrm{mg} / \mathrm{kg})$ post-operatively to reverse the Xylazine and were closely observed during recovery for signs of distress.

\subsubsection{Sample preparation}

Three rats were sacrificed at each of 4 time points $(0,6,24$, and $168 \mathrm{~h}$ post-fracture). It is crucial to understand that the $\mathrm{t}=0$ time point is not a true zero; instead, $\mathrm{t}=0$ represents the brief elapsed period of time between the occurrence of the traumatic injury and the collection of tissue samples. Rats were anesthetized intraperitoneally with Ketamine $(80-90 \mathrm{mg} / \mathrm{kg})$ and Xylazine $(10-15 \mathrm{mg} / \mathrm{kg})$. One cc of Euthasol was then administered via intracardiac puncture. Skeletal muscle tissue was harvested from the following three locations: at the site of the fracture, $1.0 \pm 0.2$ $\mathrm{cm}$ away from the site of fracture, and from the leg opposite to the fractured $\operatorname{leg}^{11}$. Samples were immediately rinsed with ice cold phosphate buffered saline (PBS), snap frozen, and stored at -80 ${ }^{\circ} \mathrm{C}$. Protein extraction was achieved using methods adapted from Hulse et al [27]. Samples were subsequently ground cryogenically and lyophilized. For analyses, 2-3 mg of lyophilized tissue sample was thawed for $10 \mathrm{~min}$ at $4{ }^{\circ} \mathrm{C}$ in cell lysis buffer (Bio-Rad, Hercules, CA) containing 20 $\mathrm{mM}$ phenylmethylsulfonyl fluoride (Sigma, St. Louis, MO). Thawed samples were then vortexed for 1-3 s and homogenized with 3 rapid pulses using an ultrasonic dismembrator. Following an additional $1-3 \mathrm{~s}$ of vortexing, samples were centrifuged at $5000 \mathrm{~g}$ for $5 \mathrm{~min}$ at $4{ }^{\circ} \mathrm{C}$. The supernatant was collected and total protein concentration was determined using the RCDC protein assay (Bio- 
Rad, Hercules, CA) according to the manufacturer's instructions. Absorbance values were determined using an Infinite M1000 plate reader (Tecan, Raleigh, NC).

\subsubsection{Analyte measurement}

Sample homogenates were diluted to a total protein concentration of $900 \mu \mathrm{g} / \mathrm{ml}$ with sample diluent (Bio-Rad, Hercules, CA). The relative abundance of total protein was determined using a Bio-plex kit containing polystyrene, nonmagnetic antibody coated beads specific for Akt, c-Jun, CREB, ERK1/2, JNK, MEK1, and p38. Phosphoproteins were assayed using the Bio-plex phosphoprotein kit containing polystyrene, non-magnetic antibody coated beads specific for the following targets phosphorylated at the indicated amino acid residues: Akt (Ser472), c-Jun (Ser63), CREB (Ser133), ERK1/2 (Thr202/Tyr204, Thr185/Tyr187), JNK (Thr183/Tyr185), MEK1 (Ser217/Ser221), p38 (Thr180/Tyr182), GSK-3 $\alpha / \beta$ (Ser21/Ser9), HSP27(Ser78), IкB $\alpha$ (Ser536), p70S6K (Thr421/Ser424), and STAT3 (Tyr705). All beads were analyzed using the Bio-Plex 200 suspension array system, along with the Pro II Wash Station (Bio-Rad, Hercules, CA), according to the manufacturer's instructions. Cytokine concentrations from a previous study [11] were measured with a Bio-Plex Pro multiplexed magnetic bead-based immunoassay reagent kit. All beads were analyzed using the Bio-Plex 200 suspension array system, along with the Pro II Wash Station (Bio-Rad, Hercules, CA), according to the manufacturer's instructions.

\subsubsection{Statistical analysis}

Data were analyzed using Prism 5 (GraphPad, San Diego, CA) and SAS JMP (Carey, NC). Protein and phosphoprotein abundances were compared to a blank subtracted intensity of the relative fluorescence intensity (RFI) measured for each antibody-coated bead. Cytokine 
concentrations $(\mathrm{pg} / \mathrm{mL})$ were derived from five-parameter logistic regression standard curves (Appendix A). Cytokine concentrations are expressed as nanogram of cytokine per gram of total protein (ng/g). Two-way analysis of variance (ANOVA) with Bonferroni's post-test was carried out using Prism 5, which identified significant differences $(\mathrm{p}<0.05)$ between each sampling location and between time points. All measurements were performed in duplicate. Data are expressed as the mean \pm standard error of the mean (SEM).

Quadratic discriminant analysis was conducted to evaluate the combined capacity of the protein, phosphoprotein, and cytokine responses [11] to predict the corresponding location and time of the injury. Using SAS JMP, all measured responses were cast as covariates (Y) and either location or time was assigned as the classification category (X); the Shrink Covariances option was applied to account for the different covariance within the categories. This analysis included 36 observations ( 3 rats for each of the 3 locations and 4 time points) for 25 different covariates. The mean of the covariates in a specific group was calculated, along with $95 \%$ confidence levels. Finally, biplot rays were determined that indicate how each covariate fits into the canonical space, with the direction signifying the degree of association within that space.

Significant pairwise correlations $(\mathrm{p}<0.05)$ between cytokine and total protein/phosphoprotein responses were determined using SAS JMP. This analysis investigated the association of total protein levels and phosphorylation responses with cytokine production at each time point and sample location. Data used for spatial correlation analysis consisted of values from every time point $(0,6,24$, and $168 \mathrm{~h})$, with a maximum $\mathrm{N}$ of 12 . Data used for temporal analysis included values from all sample locations (At fracture, 1-cm away, and uninjured leg), with a 
maximum $\mathrm{N}$ of 9 . Outliers, or data points that were driving the correlation were identified and eliminated using boundaries set by the interquartile range.

\subsection{Results}

\subsubsection{Spatial and temporal total protein levels}

Relative levels of total protein, regardless of the phosphorylation state (phosphorylated or unphosphorylated) are presented in Figure 2.1 for 7 targets: Akt, c-Jun, CREB, ERK1/2, JNK, MEK1, and p38. Immediately following injury (at hour 0), total Akt and ERK1/2 levels were significantly higher $(\mathrm{p}<0.05)$ in tissue taken from the uninjured leg in comparison to tissue at the fracture site and to the tissue sampled 1-cm away from the fracture. No other proteins exhibited significant differences among the three sample sites at hour 0 . At $6 \mathrm{~h}$ post-fracture, expression of only p38 was significantly different at the fracture site compared to the amount found in the uninjured tissue. Spatial variances in p38 expression continued to persist at $24 \mathrm{~h}$ post-fracture, when the level at the fracture site was significantly lower than levels found in both 1-cm away and uninjured tissue samples. MEK1 was additionally observed to exhibit a spatial gradient $24 \mathrm{~h}$ after injury, with significantly lower levels at the fracture site compared to tissue 1-cm away and in the uninjured leg.

The highest number of significant differences in protein expression among different sampling locations was observed $168 \mathrm{~h}$ post-fracture. Expression levels of Akt and ERK1/2 were significantly higher at the site of injury in comparison to the uninjured leg, while c-Jun, CREB, JNK, and p38 levels were all statistically higher at the site of fracture in comparison to both 1-cm 
away and uninjured tissue samples. It was noted that the spatial pattern observed at $168 \mathrm{~h}$ was opposite the trend observed at the earlier time points, with protein expression levels being highest at the site of fracture.

\subsubsection{Spatial and temporal response of phosphorylated proteins}

To further examine the contribution of the selected proteins, phosphorylation levels were determined for the following targets: Akt, c-Jun, CREB, ERK1/2, JNK, MEK1, p38 (Figure 2.2). In addition, phosphorylation responses of GSK-3 $\alpha / \beta, \mathrm{HSP} 27, \mathrm{I} \kappa \mathrm{B} \alpha, \mathrm{p} 70 \mathrm{~S} 6 \mathrm{~K}$, and STAT3 (Figure 2.3) were also measured. No statistically significant spatial differences for phosphorylated JNK or I $\mathrm{B} \alpha$ were observed at any time point. At 0 hour post-fracture, phosphorylated levels of Akt, cJun, ERK1/2, GSK-3 $/ / 3$, HSP27, p70S6K, and STAT3 were significantly higher in tissue taken from the uninjured leg compared to both tissue samples collected at the fracture site and 1-cm away from the fracture. In contrast, CREB phosphorylation levels only differed between the uninjured and 1-cm away site, while phosphorylation of MEK1 and p38 did not present any spatial differences.

At the later time points, the number of phosphorylated proteins with significant spatial differences decreased. Only Akt, GSK-3 $\alpha / 3$, and MEK1 displayed spatial gradients at 6 hours postfracture, with the lowest degree of phosphorylation at the site of fracture for Akt and GSK-3 $\alpha / \beta$. At 24 hours post-fracture, GSK-3 $3 / \beta$ continued to present spatial phosphorylation differences, along with a newly present $\mathrm{p} 38$. Phosphorylation levels of both GSK-3 $\alpha / \beta$ and p38 were lowest at the site of fracture during this time. The distribution of phosphorylated proteins at the final time point (168 hours) deviated from the pattern observed at the earlier times. While most of the 
gradients revealed lower levels of phosphorylated protein at the fracture site at 0,6 , and 24 hours, the levels of phosphorylated MEK1 and p70S6K were highest at the site of tissue injury at 168 hours post-fracture.
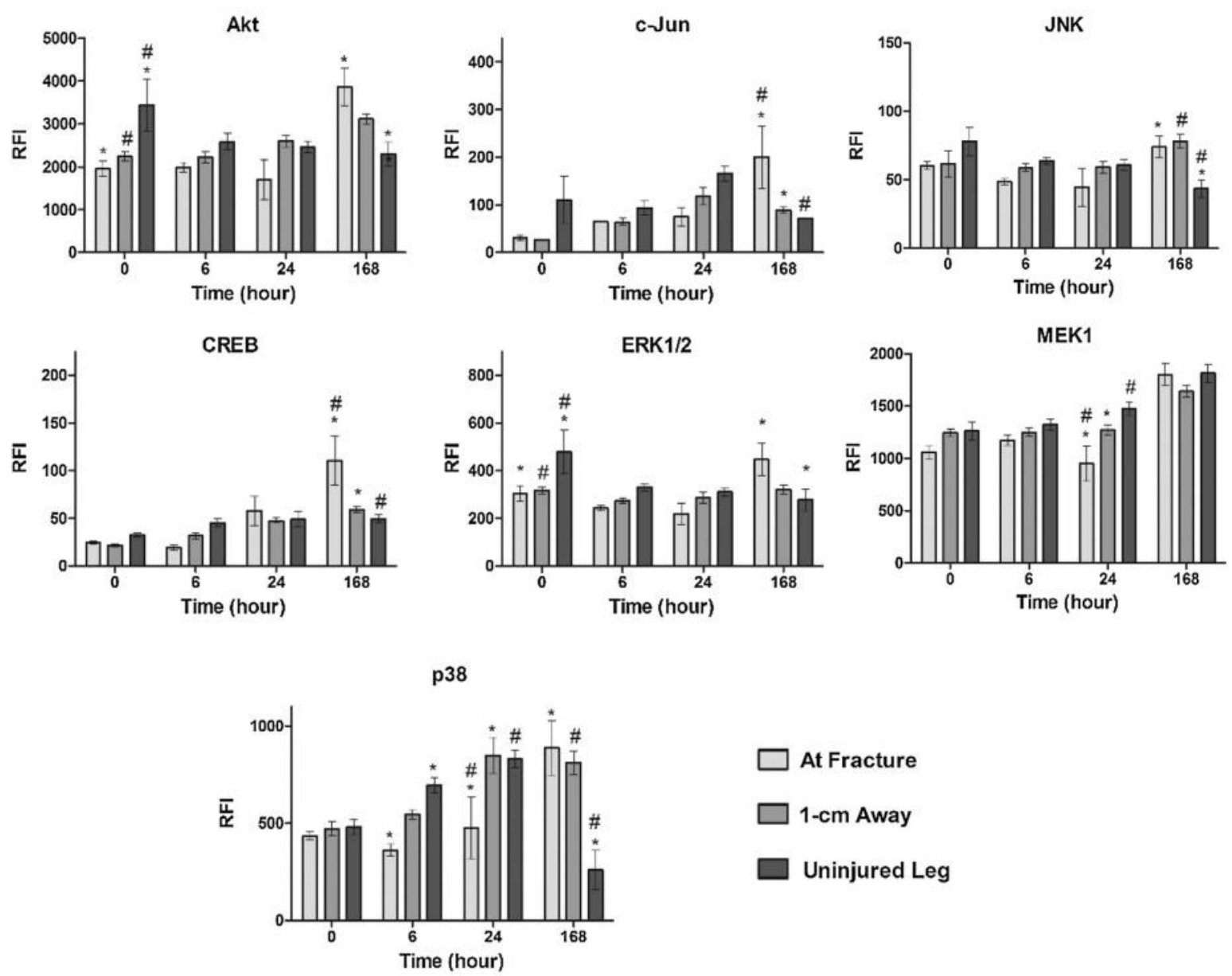

Figure 2.1. Total protein concentration measured across time and location in response to a traumatic injury. Relative fluorescence intensity (RFI) associated with total protein concentrations of the following proteins were assayed across four time points and three different locations following the femur fracture: Akt, c-Jun, CREB, ERK1/2, JNK, MEK1, and p38. Statistically significant differences $(\mathrm{p}<0.05)$ in protein concentration between different locations are marked with matching symbols ( $*$ or \#). Error bars reflect \pm standard error of the mean. 

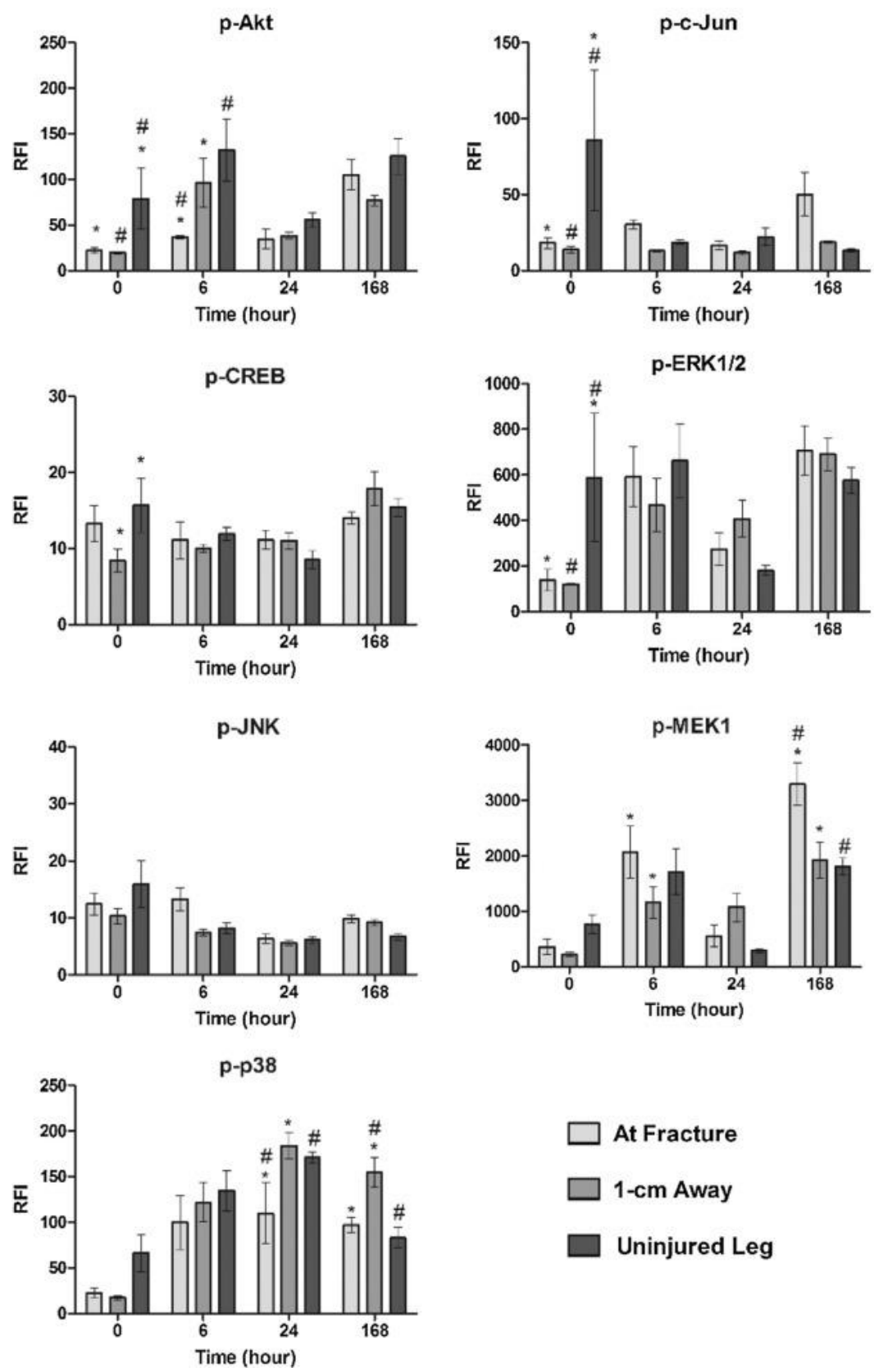

Figure 2.2. Phosphorylation levels measured across time and location in response to a traumatic injury. Levels of phosphorylated protein of Akt, c-Jun, CREB, ERK1/2, JNK, MEK1, and p38 were determined for each time point and location. Concentrations are expressed as relative fluorescence intensity (RFI). Statistically significant differences $(p<0.05)$ in protein concentration between different locations are marked with matching symbols $(*$ or \#). Error bars reflect \pm standard error of the mean. 

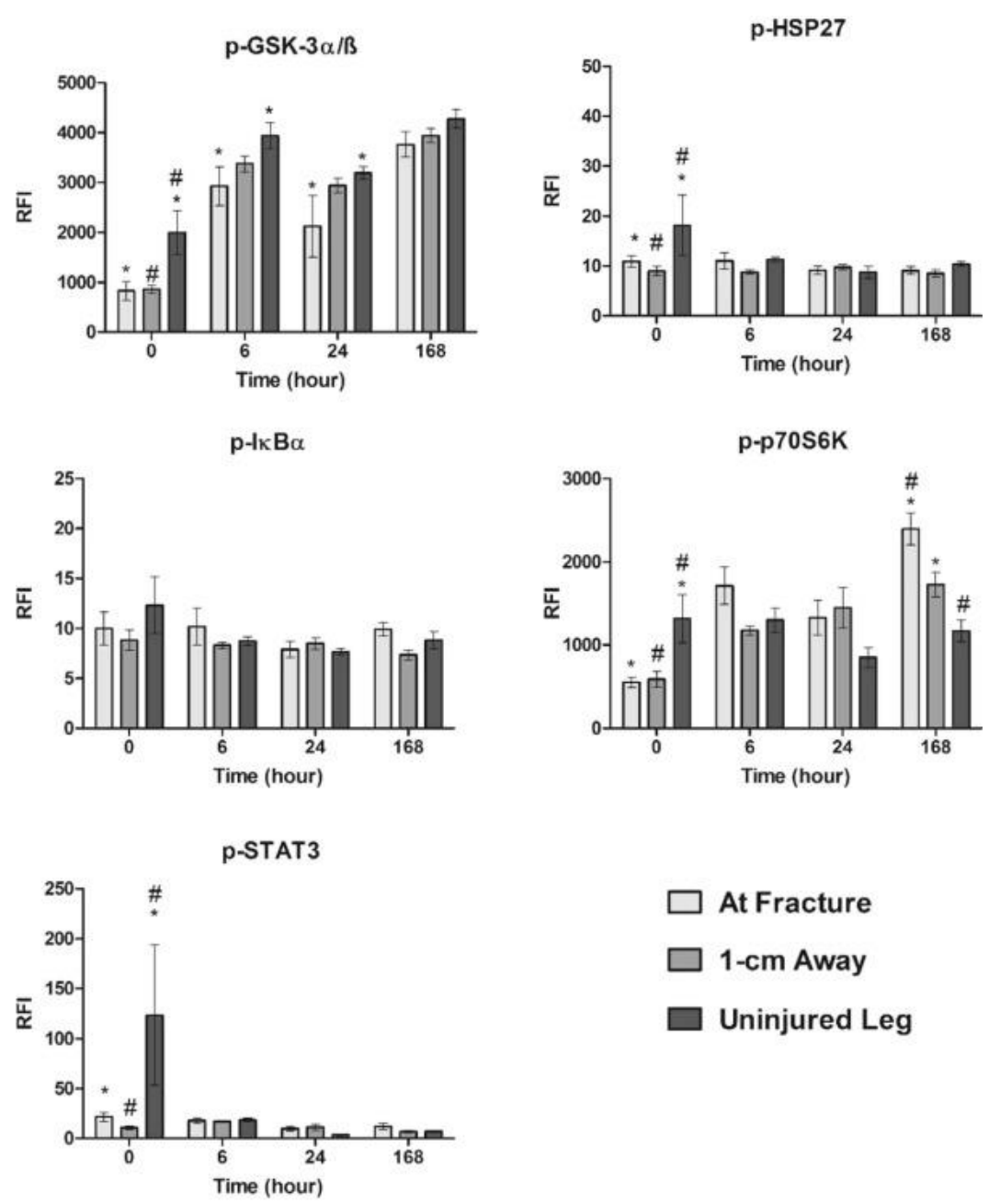

Figure 2.3. Phosphorylation levels measured across time and location in response to a traumatic injury. Levels of phosphorylated protein of GSK-3 $\alpha / 3$, HSP27, IкB $\alpha$, p70S6K, STAT3 were determined for each time point and location. Concentrations are expressed as relative fluorescence intensity (RFI). Statistically significant differences $(\mathrm{p}<0.05)$ in protein concentration between different locations are marked with matching symbols $(*$ or \#). Error bars reflect \pm standard error of the mean.

\subsubsection{Spatial and temporal cytokine concentrations}

Figure 2.4 shows an adapted figure from Currie, et al. demonstrating spatial and temporal differences of cytokine responses following the same traumatic injury as described in this paper [11]. Concentrations of the following cytokines were measured: IL-1 $\alpha$, IL-1ß, IL2, IL6, TNF- $\alpha$, 
and MIP-1 $\alpha$. IL-2 was the sole cytokine with any spatial differences at $t=0$, but did not exhibit any significant differences at the other time points. IL-1ß and IL6 displayed strong significant changes in spatial response only at $\mathrm{t}=6$, while IL- $1 \alpha$ represented the single cytokine to express spatial alterations at $\mathrm{t}=24$. TNF- $\alpha$, and MIP- $1 \alpha$ did not exhibit any statistically significant spatial nor temporal differences.
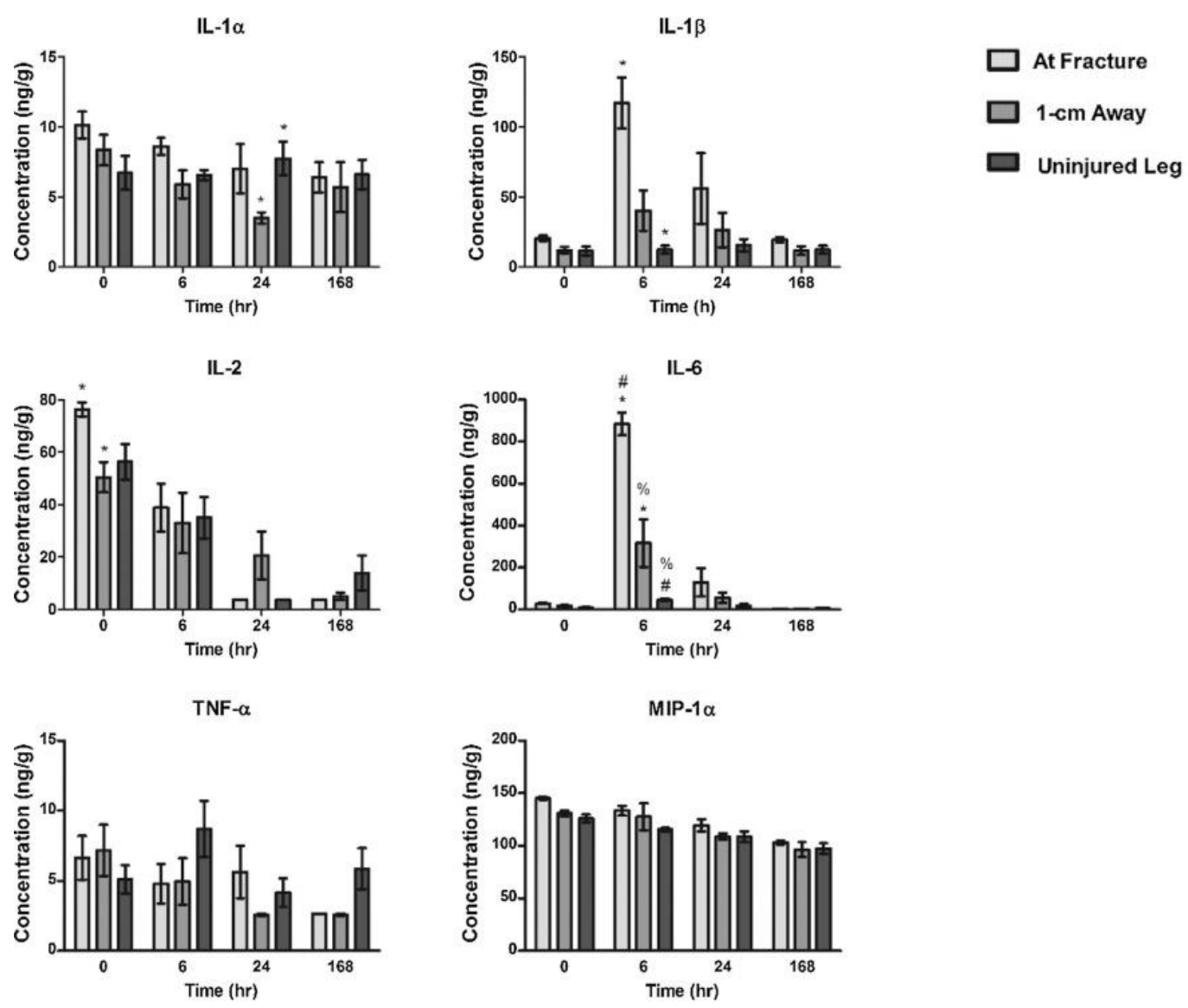

Figure 2.4 Cytokine levels measured across time and location in response to a traumatic injury. Concentrations (ng/g) of IL-1 $\alpha$, IL-1ß, IL2, IL6, TNF- $\alpha$, and MIP-1 $\alpha$ were determined for each time point and location. Statistically significant differences $(\mathrm{p}<0.05)$ of cytokine concentration between different locations at each time point are marked with matching symbols (*, \#, or \%). Error bars reflect \pm standard error of the mean. Figure reprinted from Cytokine, 66, H.N. Currie, M.S. Loos, J.A. Vrana, K. Dragan, J.W. Boyd, Spatial cytokine distribution following traumatic injury, 112-118, Copyright 2014, with permission from Elsevier. 


\subsubsection{Quadratic discriminant analysis used to predict location and time of injury}

Quadratic discriminant analysis was performed using all measured responses: total protein concentration (Akt, c-Jun, CREB, ERK1/2, JNK, MEK1, p38), phosphorylation levels (p-Akt, p-

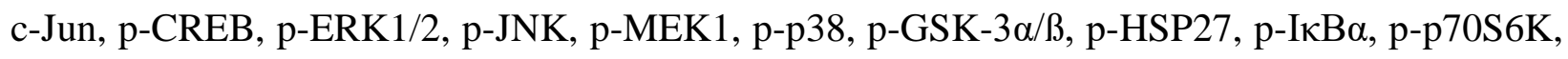
and p-STAT3), and cytokine concentrations determined in a previous study (IL-1 $\alpha$, IL-1ß, IL2, IL6, TNF- $\alpha$, and MIP-1 $\alpha$ ) [11]. This analysis classified the combined observed responses into predetermined groups (location or time) by plotting each collective observation against two canonical coordinates. In Figures 2.5 and 2.6, the location and time of injury were predicted based on the responses associated with each sample, respectively. In total, discriminant analysis misclassified only 10 observations out of 36 for location predictions and none for time predictions: 4 observations that were derived from samples taken from at the site of injury were predicted as belonging to the 1-cm away group and 6 observations that were associated with samples from the other uninjured leg were incorrectly grouped in the 1-cm away group. 


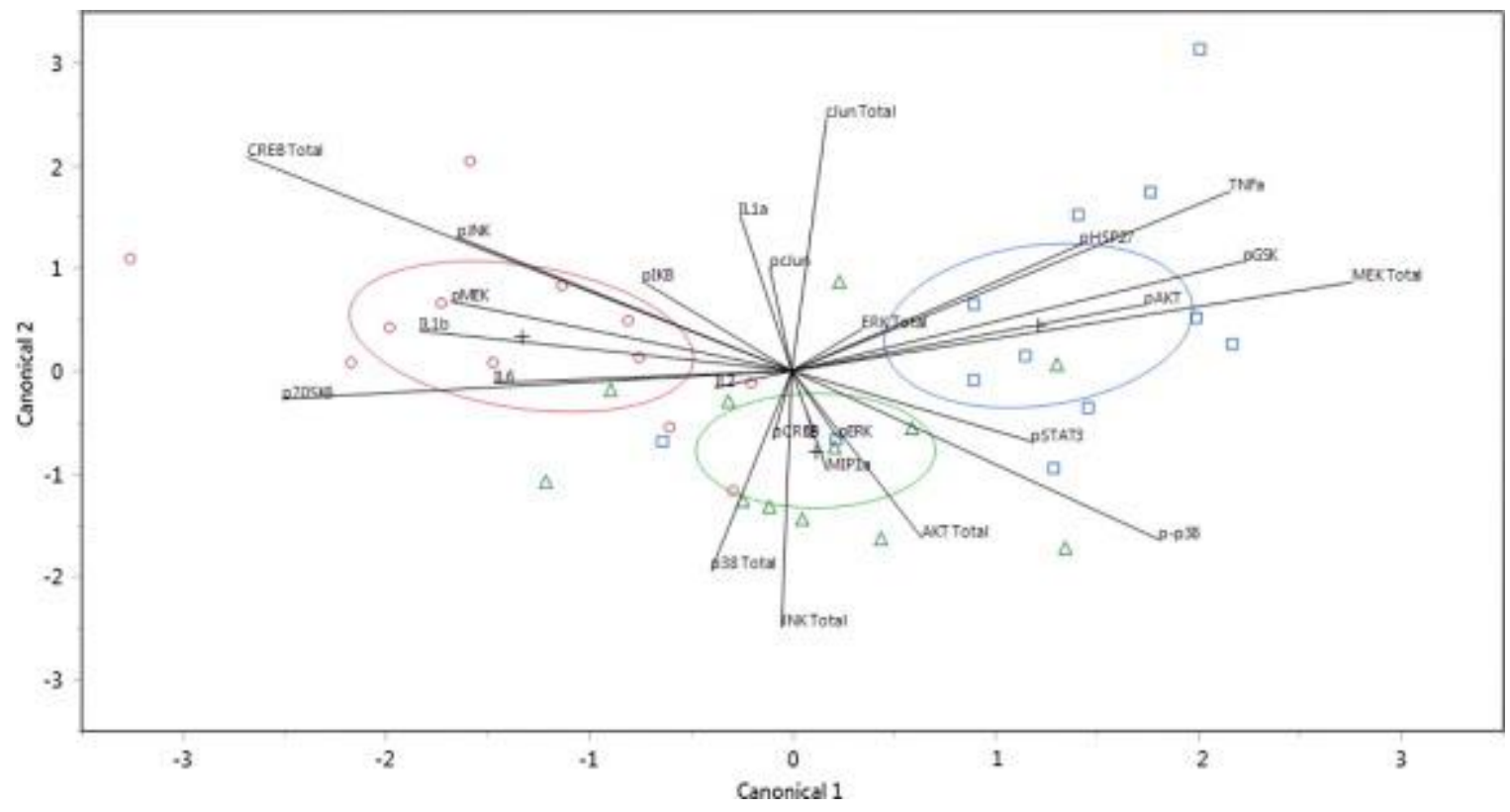

Figure 2.5. Canonical scores plot for the identification of the location of injury. Canonical scores for each covariate calculated by quadratic discriminant analysis are plotted. The different locations of injury, at the site (red circles), 1-cm away (green triangles), and on the other uninjured leg (blue squares), were identified. The + signifies the mean of the covariates in each group. The ellipses represent a 95\% confidence level and the biplot rays describe the degree of association of a certain covariate with the canonical variables. 


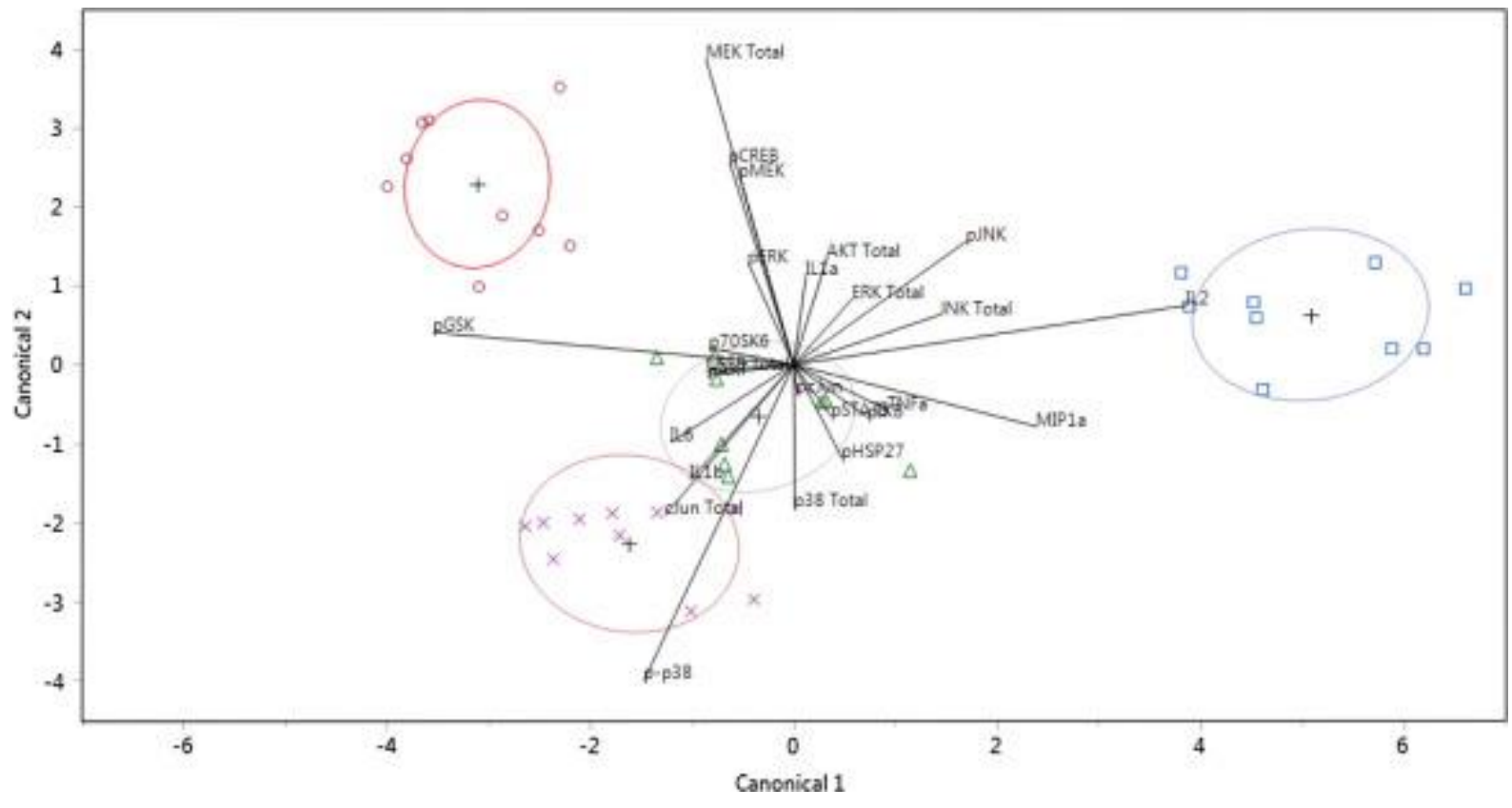

Figure 2.6. Canonical scores plot for the identification of the time of injury. Canonical scores for each covariate calculated by quadratic discriminant analysis are plotted. The different times of injury, 0 (blue squares), 6 (green triangles), 24 (purple X's) and 168 (red circles) h were identified. The + signifies the mean of the covariates in each group. The ellipses represent a $95 \%$ confidence level and the biplot rays describe the degree of association of a certain covariate with the canonical variables. 


\subsubsection{Correlation between cytokine and phosphoprotein response}

The results of the correlation analysis between protein and phosphorylation responses in

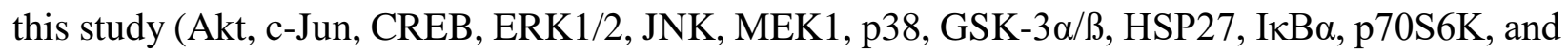
STAT3) and cytokine responses from a previous study (IL-1 $\alpha$, IL-1ß, IL2, IL6, TNF- $\alpha$, and MIP-

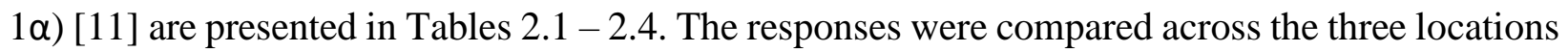
and at each of the four time points. It is important to note that these correlations are possible because the tissue samples are from the same animals, locations, and time points as described in Currie, 2014. Significant spatial correlations (Table 2.1 and 2.2) identified certain cytokines and phosphoproteins that responded similarly at particular locations. At the site of the fracture, MIP$1 \alpha$ was a noted contributor with negative correlations to four proteins (CREB, MEK, c-Jun, and Akt) and phosphorylation responses of five phosphoproteins (Akt, p70S6K, GSK-3 $\alpha / 3$, MEK1, and ERK1/2). Although there were no statistically significant spatial nor temporal differences for MIP-1 $\alpha$, a general decrease in concentration can be seen with increasing time. Moving 1-cm away from the site of injury, IL2 and IL-1ß joined MIP-1 $\alpha$ in being negatively correlated to a larger collection of proteins and phosphoproteins with the exception of two positive correlations between IL2/IкB $\alpha$ and IL-1ß/STAT3 (Table 2.2). More positive correlations were identified in the sample from the uninjured leg: MEK1 levels were negatively correlated to cytokines IL6 and IL2 measured in the uninjured leg, while Akt levels were positively correlated to one cytokine (IL-1 $\alpha$ ). All positive correlations between phosphorylation and cytokine responses (IL2/STAT3, IL2/JNK, TNF- $\alpha /$ ERK1/2, TNF- $\alpha /$ p70S6K) were also found in the uninjured leg. 
Table 2.1. Results of spatial cytokine-total protein response correlation analysis. Pearson correlation coefficients $(r)$ and significance values $(p)$ for associated cytokines and total protein levels across all sampling locations are listed. $N=12$ and any outliers driving correlations were identified and removed.

\begin{tabular}{cccc}
\hline Cytokine & Total Protein & Correlation (r) & $\mathrm{p}$ \\
\hline At Fracture & & & \\
MIP-1 $\alpha$ & CREB & -0.7570 & 0.0112 \\
MIP-1 $\alpha$ & MEK1 & -0.7740 & 0.0086 \\
MIP-1 $\alpha$ & c-Jun & -0.7447 & 0.0135 \\
MIP-1 $\alpha$ & Akt & -0.6485 & 0.0425 \\
IL2 & MEK1 & -0.7521 & 0.0121 \\
TNF- $\alpha$ & MEK1 & -0.7728 & 0.0088 \\
IL-1 $\alpha$ & MEK1 & -0.6630 & 0.0367 \\
IL-1 $\alpha$ & JNK & -0.6302 & 0.0377 \\
IL-1 $\beta$ & ERK1/2 & -0.6498 & 0.0420 \\
& & & \\
1-cm Away & & & 0.0109 \\
MIP-1 $\alpha$ & CREB & -0.7023 & 0.0458 \\
MIP-1 $\alpha$ & p38 & -0.5849 & 0.0025 \\
IL2 & CREB & -0.7846 & 0.0447 \\
IL2 & Akt & -0.5873 & \\
& & & 0.0091 \\
Uninjured Leg & & -0.7139 & 0.0415 \\
IL6 & MEK1 & -0.5944 & 0.0430 \\
IL2 & MEK1 & 0.6174 & \\
IL-1 $\alpha$ & Akt & & \\
\hline
\end{tabular}


Table 2.2. Results of spatial cytokine-phosphorylated protein response correlation analysis. Pearson correlation coefficients (r) and significance values ( $p$ ) for associated cytokines and phosphorylation responses across all sampling locations are listed. $\mathrm{N}=12$ and any outliers driving correlations were identified and removed.

\begin{tabular}{cccc}
\hline Cytokine & Phosphoprotein & Correlation $(\mathrm{r})$ & $\mathrm{P}$ \\
\hline At Fracture & & & \\
MIP-1 $\alpha$ & p-Akt & -0.8639 & 0.0013 \\
MIP-1 $\alpha$ & p-p70S6 & -0.8297 & 0.0016 \\
MIP-1 $\alpha$ & p-GSK-3 $/ \beta$ & -0.8161 & 0.0022 \\
MIP-1 $\alpha$ & p-MEK1 & -0.6863 & 0.0197 \\
MIP-1 $\alpha$ & p-ERK1/2 & -0.6515 & 0.0299 \\
& & & \\
1-cm Away & & & \\
MIP-1 $\alpha$ & p-Akt & -0.6449 & 0.0321 \\
MIP-1 $\alpha$ & p-GSK-3 $\alpha / \beta$ & -0.5807 & 0.0477 \\
IL2 & p-p70S6 & -0.7543 & 0.0046 \\
IL2 & p-Akt & -0.7547 & 0.0073 \\
IL2 & p-IKB $\alpha$ & 0.6273 & 0.0388 \\
IL2 & p-GSK-3 $/ \beta$ & -0.5913 & 0.0429 \\
IL-1 $\beta$ & p-STAT3 & 0.7341 & 0.0066 \\
& & & \\
Uninjured Leg & & & \\
IL2 & p-STAT3 & 0.6633 & 0.0261 \\
IL2 & p-JNK & 0.6416 & 0.0333 \\
TNF $\alpha$ & p-ERK1/2 & 0.8460 & 0.0005 \\
TNF $\alpha$ & & 0.7598 & 0.0041 \\
\hline
\end{tabular}


Table 2.3. Results of temporal cytokine-total protein response correlation analysis. Pearson correlation coefficients $(r)$ and significance values $(p)$ for associated cytokines and total protein levels across all time points are listed. $N=9$ and any outliers driving correlations were identified and removed.

\begin{tabular}{|c|c|c|c|}
\hline Cytokine & Total Protein & Correlation (r) & $\mathrm{P}$ \\
\hline \multicolumn{4}{|l|}{$\mathbf{T}=\mathbf{0}$} \\
\hline IL6 & p38 & -0.7923 & 0.0109 \\
\hline IL6 & c-Jun & -0.8543 & 0.0144 \\
\hline IL6 & MEK1 & -0.6888 & 0.0402 \\
\hline IL-1 $\alpha$ & c-Jun & -0.8885 & 0.0075 \\
\hline $\mathrm{TNF} \alpha$ & p38 & -0.7761 & 0.0139 \\
\hline IL2 & p38 & -0.7291 & 0.0258 \\
\hline IL2 & MEK1 & -0.7252 & 0.0270 \\
\hline MIP-1 $\alpha$ & c-Jun & -0.8098 & 0.0273 \\
\hline \multicolumn{4}{|l|}{$T=6$} \\
\hline IL6 & p38 & -0.8690 & 0.0024 \\
\hline IL6 & ERK1/2 & -0.8733 & 0.0046 \\
\hline IL6 & JNK & -0.8410 & 0.0089 \\
\hline IL-1 $\beta$ & p38 & -0.8507 & 0.0036 \\
\hline IL-1 $\beta$ & JNK & -0.8225 & 0.0122 \\
\hline IL-1 $\beta$ & ERK1/2 & -0.8865 & 0.0033 \\
\hline $\mathbf{T}=24$ & No Correlations & & \\
\hline$T=168$ & No Correlations & & \\
\hline
\end{tabular}


Table 2.4. Results of temporal cytokine-phosphorylated protein response correlation analysis. Pearson correlation coefficients $(r)$ and significance values $(p)$ for associated cytokines and phosphorylation responses across all time points are listed. $N=9$ and any outliers driving correlations were identified and removed.

\begin{tabular}{cccc}
\hline Cytokine & Phosphoprotein & Correlation $(\mathrm{r})$ & $\mathrm{P}$ \\
\hline T=0 & & & \\
IL-1 $\alpha$ & p-GSK-3 $\alpha / \beta$ & -0.7709 & 0.0251 \\
IL-1 $\alpha$ & p-p70S6 & -0.7538 & 0.0308 \\
IL6 & p-CREB & 0.7121 & 0.0314 \\
IL2 & p-CREB & 0.6966 & 0.0371 \\
IL2 & p-MEK1 & 0.6834 & 0.0424 \\
& & & \\
T=6 & & & \\
IL-1 $\alpha$ & p-c-Jun & 0.8207 & 0.0125 \\
IL-1 $\beta$ & p-JNK & 0.7943 & 0.0186 \\
MIP-1 $\alpha$ & p-Akt & -0.7101 & 0.0484 \\
& & & \\
T=24 & & & 0.0035 \\
IL6 & p-HSP27 & 0.9184 & 0.0349 \\
IL6 & p-CREB & 0.7892 & 0.0286 \\
IL-1 $\beta$ & p-HSP27 & 0.8060 & \\
& & & \\
T=168 & No Correlations & & \\
\hline
\end{tabular}


Temporal correlation analysis between total protein and cytokine levels (Table 2.3) identified eight significant pairs at 0 hours post fracture (IL6/p38, IL6/c-Jun, IL6/MEK1, IL-1 a/cJun, TNF- $\alpha / \mathrm{p} 38, \mathrm{IL} 2 / \mathrm{p} 38, \mathrm{IL} 2 / \mathrm{MEK} 1$, and MIP-1 $\alpha / \mathrm{c}-\mathrm{Jun})$, six pairs at 6 hours post fracture (IL6/p38, IL6/ERK1/2, IL6/JNK, IL-1ß/p38, IL-1ß/JNK, and IL-1ß/ERK1/2), and no correlations at 24 and 168 hours post fracture. Temporal correlation analysis between cytokine and phosphoprotein responses (Table 2.4) determined five pairwise correlations at 0 hours postfracture (IL-1 $\alpha /$ GSK-3 $\alpha / \beta$, IL-1 $\alpha /$ p70S6K, IL6/CREB, IL2/CREB, and IL2/MEK1), three correlations at 6 hours (IL-1 $\alpha / \mathrm{c}-\mathrm{Jun}, \mathrm{IL}-1 \beta / \mathrm{JNK}$, and MIP-1 $\alpha / \mathrm{Akt}$ ), three correlations at 24 hours (IL6/HSP27, IL6/CREB, and IL-1ß/HSP27), and zero correlations at 168 hours. All correlations between protein and cytokine levels were negative, while a mixture of positive and negative correlations was identified for phosphorylation and cytokine responses.

\subsection{Discussion}

Spatial and temporal differences in phosphorylation mediated signaling in response to a traumatic injury is still an unfamiliar area within the field of wound healing mechanisms. Injury and repair biomarkers, including cytokines, neutrophils, leukocytes, and growth factors are some of the more characterized aspects, but piecing all of the components together is a necessity to improve our understanding of the wound healing process. This study provides a depiction of the intracellular signaling that occurs as a result of a reproducible leg fracture model, in an effort to deliver an enhanced understanding of the healing process and treatment options. Defining location and time dependent differences in phosphoprotein response, along with identifying associations with cytokine production, was the focus of this investigation. 
Measurement of the total protein levels (phosphorylated and unphosphorylated) identified several proteins with a suggested significance at certain time points and locations (Figure 2.1). In particular, a differential distribution of Akt and ERK protein expression at the different sampling locations was observed. Akt and ERK1/2 pathways are notably recognized as key contributors of cell growth and survival $[28,29]$ and the data in this study demonstrated that they become more active, through increased expression, during the later stages in the healing process when new tissue formation and remodeling is likely to occur. Other proteins (c-Jun, CREB, JNK, MEK1, and p38) also exhibited significant spatial differences at later time points. In agreement with previous studies, MAPK signaling pathways involving ERK1/2, MEK1, and JNK have been reported to respond to injuries induced in skeletal muscles [30].

While assessing protein expression can offer substantial insight into the healing mechanism, it is necessary to evaluate the associated post-translational activity since alterations in protein abundance are not as immediate as phosphorylation processes. All of the measured total proteins were evaluated for phosphorylation responses, and in an effort to investigate a larger collection of phosphorylation changes, GSK-3 $\alpha / \beta$, HSP27, IкB $\alpha, \mathrm{p} 70 \mathrm{~S} 6 \mathrm{~K}$, and STAT3 were also monitored (Figures 2.2 and 2.3). Similar to the response of total protein expression, the results indicate that protein phosphorylation at early time points ( 0 and 6 hours post injury) is generally decreased at the fracture site and surrounding regions, as there were no instances where phosphorylated protein levels were lower in the uninjured tissue. This decrease in phosphorylation may be due to a combination of increased apoptosis [31], decreased cellular regulation over kinase activity [32], injury induced hypoxia [33] and, in contrast, hyperoxia [34], or ATP availability [35]. For proteins that experienced increases in phosphorylation at or near the site of fracture, this typically occurred 
at the later time points, suggesting prominent roles for these proteins in the later stages of mediating repair. It is important to note that a decreased level in phosphorylation does not necessarily equate to a decrease in activity of that protein. For instance, a decrease in Ser-21/9 phosphorylation on GSK-3 $\alpha / \beta$ corresponds to an increase in GSK-3 $\alpha / \beta$ activity [36]. Two of the measured phosphoproteins, JNK and $\mathrm{I} \kappa \mathrm{B} \alpha$, did not exhibit temporal or spatial differences in phosphorylation, but this is only true at the specific time points selected in this study.

In order to begin to understand the integrated cellular response to traumatic injury and follow-on wound healing, it is also useful to examine the relationships and coordinated efforts of related proteins. For example, p70S6K is implicated as a downstream target of the Akt/mammalian target of rapamycin (mTOR) signaling pathway following its activation during muscle remodeling [37]. The Akt signaling pathway is also known to promote protein synthesis to increase muscle mass through the activation of p70S6K [38]. In agreement with these previous studies, this work shows that at the last time point measured (168 hours post-fracture), at the site of fracture, the phosphorylation levels of Akt had increased and returned to comparable levels as found in the uninjured leg. At the same time, phosphorylation levels of p70S6K were also significantly amplified at the site of fracture, indicating the increased phosphorylation levels of both proteins may signify crucial times of muscle repair. GSK-3 $\alpha / \beta$, another downstream target of the Akt signaling pathway, is known to be an extremely important mediator of muscle tissue plasticity, and has been observed to increase in activity in skeletal muscle resulted from burn injuries [39]. Akt inhibits GSK-3 $\alpha / \beta$ activity directly by phosphorylating Ser-21 and Ser-9 on the $\alpha$ and $\beta$ isoforms, respectively [40]. At the 0 time point, Akt protein expression and phosphorylation along with GSK-3 $\alpha / \beta$ phosphorylation seemed to be in accord, with significantly lower levels at the 
fracture site in comparison to the uninjured leg. These results suggest GSK-3 $\alpha / \beta$ phosphorylation is associated with Akt during early periods after the fracture.

One of the most well characterized aspects of the response to traumatic injury is the regulatory role of cytokines. Cytokine production has been shown to reflect the degree of tissue trauma, and temporal changes in cytokine concentrations have been shown to correlate with adverse early post-trauma implications such as acute respiratory distress syndrome (ARDS) [41], multiple organ failure (MOF) [42], and mortality [43]. In previous studies, correlations between repair biomarkers and phosphoprotein responses have been identified. For instance, the degree of GSK-3 $\alpha / \beta$ phosphorylation is reported to be strongly correlated to levels of $\beta$-catenin protein, a crucial regulator of wound healing [44]. p38 is also known to respond to cytokines and various types of stress [45] and has been shown to increase dramatically in injured human muscle [46]. In the context of wound healing, cytokine expression and phosphorylation levels of associated proteins have been linked together [47], implicating the importance of integrated extracellular and intracellular signaling during crucial periods of repair. Cytokine responses (Figure 2.4) from previous research also exhibited a spatiotemporal distribution as a result of the same traumatic injury. In this study, discriminant analysis was conducted using protein, phosphorylation, and cytokine levels in order to evaluate all three responses, collectively, for all locations and time points. Correlation analysis was also performed to investigate the pairwise spatiotemporal relationships among total protein, phosphoproteins, and cytokines.

Discriminant analysis has previously been demonstrated to successfully predict adverse outcomes, such as ligament injuries [48], cardiotoxicity [49], and asphyxia [50]. Instead of 
predicting adverse outcomes, the observed responses reported in this study were used to distinguish between different locations and times of injury [Figures 2.5 and 2.6]. Classification of the observations based on time yielded a prediction rate of $100 \%$, while classification based on location yielded a prediction rate of about $72 \%$, incorrectly placing 10 out of 36 observations into the wrong groups. The misclassified observations only occurred for location predictions: 4 observations from the site of injury group were predicted as belonging to the 1-cm away group and 6 observations from other uninjured leg group were incorrectly grouped in the 1-cm away group. These misclassifications were not unexpected because although the 1-cm away samples are technically uninjured tissue, the surrounding area near the site of injury still experienced indirect impacts (i.e. inflammation) from the traumatic injury. These results indicate that there is a strong temporal and spatial distinction in tissue response following a traumatic injury that can be observed with both intracellular (total protein, phosphorylation) and extracellular (cytokine) responses, further confirming that a distinct spatiotemporal gradient for all three responses exist. The promising predictive rates also suggest a coordinated effort of proteins, phosphorylation signaling, and cytokine regulation in response to the injury since each observation (data point in the canonical plot) integrated all measurements of the dataset.

Finally, significant correlated pairings of phosphoprotein and cytokine responses were discerned (Tables 2.1-2.4) in order to monitor how associated pairs may be altered over time and location. As a primary marker of injury, cytokines may be critical for initiating certain processes, such as protein expression or phosphorylation, especially in cases of traumatic tissue injury. The absence and/or development of correlations may be indicative of the progression of the healing process, or lack thereof. For instance, all correlations between total protein and cytokines were 
negative at the two early time points, suggesting some form of ordered regulation. After 6 hours, zero correlations were observed, indicating a drastic change in cytokine production, protein expression, or both. While these results offer a glimpse of the tightly coordinated signaling efforts at distinct phases of repair, we believe that a more comprehensive modeling effort of spatiotemporal responses should be undertaken in order to improve our understanding of this fundamental process.

\subsection{Conclusions}

The vast majority of studies investigating the specific roles of proteins under injurious inflammatory inducing conditions are performed in vitro, typically focusing on the up or down regulation of a few select proteins. The results presented here are unique in that they reflect the entire tissue response to trauma. Overall, the data indicate that there are observable significant spatial differences in the levels of phosphorylated proteins following a traumatic injury. This is the first study to analyze the levels of phosphorylated proteins in tissue samples at different distances from a traumatic injury. It provides evidence that monitoring phosphoproteins could potentially be used to spatially assess the state of injured tissue. Additionally, exploring the integration of phosphoprotein and cytokine signaling roles under conditions of distress offers insight into the healing mechanism. This information may potentially be helpful in adapting or refining wound treatment procedures, such as the debridement of non-viable tissue, in order to prevent additional adverse outcomes resulting from possible treatment complications. 


\subsection{Acknowledgements}

This research was conducted with support from a grant awarded by The Brodie Discovery and Innovation Fund.

\subsection{References}

[1] Gurtner GC, Werner S, Barrandon Y, Longaker MT. Wound repair and regeneration. Nature. 2008;453:314-21.

[2] Velnar T, Bailey T, Smrkoli V. The Wound Healing Process: an Overview of the Cellular and Molecular Mechanisms. Journal of International Medical Research. 2009;37:1528-42.

[3] Witte MB, Barbul A. General principles of wound healing. Surgical Clinics of North America. 1997;77:509.

[4] Broughton G, 2nd, Janis JE, Attinger CE. The basic science of wound healing. Plast Reconstr Surg. 2006;117:12s-34s.

[5] Lee K, Silva EA, Mooney DJ. Growth factor delivery-based tissue engineering: general approaches and a review of recent developments. J R Soc Interface. 2011;8:153-70.

[6] Akita S. Surgical Debridement. In: Skin Necrosis: Springer Vienna; 2015. p. 257-63.

[7] Gerstenfeld LC, Cullinane DM, Barnes GL, Graves DT, Einhorn TA. Fracture healing as a post-natal developmental process: molecular, spatial, and temporal aspects of its regulation. J Cell Biochem. 2003;88:873-84.

[8] Rittie L, Perbal B, Castellot JJ, Jr., Orringer JS, Voorhees JJ, Fisher GJ. Spatial-temporal modulation of $\mathrm{CCN}$ proteins during wound healing in human skin in vivo. Journal of cell communication and signaling. 2011;5:69-80. 
[9] Kibe Y, Takenaka H, Kishimoto S. Spatial and temporal expression of basic fibroblast growth factor protein during wound healing of rat skin. British Journal of Dermatology. 2000;143:720-7.

[10] Gillitzer R, Goebeler M. Chemokines in cutaneous wound healing. J Leukoc Biol. 2001;69:513-21.

[11] Currie HN, Loos MS, Vrana JA, Dragan K, Boyd JW. Spatial cytokine distribution following traumatic injury. Cytokine. 2014;66:112-8.

[12] Hunter T. Protein kinases and phosphatases: the yin and yang of protein phosphorylation and signaling. Cell. 1995;80:225-36.

[13] Viatour P, Merville MP, Bours V, Chariot A. Phosphorylation of NF-kappaB and IkappaB proteins: implications in cancer and inflammation. Trends Biochem Sci. 2005;30:43-52.

[14] Payne J, Gong H, Trinkaus-Randall V. Tyrosine phosphorylation: a critical component in the formation of hemidesmosomes. Cell Tissue Res. 2000;300:401-11.

[15] Kasza KE, Farrell DL, Zallen JA. Spatiotemporal control of epithelial remodeling by regulated myosin phosphorylation. Proc Natl Acad Sci U S A. 2014;111:11732-7.

[16] Richards TS, Dunn CA, Carter WG, Usui ML, Olerud JE, Lampe PD. Protein kinase C spatially and temporally regulates gap junctional communication during human wound repair via phosphorylation of connexin43 on serine368. J Cell Biol. 2004;167:555-62.

[17] Noshita N, Lewen A, Sugawara T, Chan PH. Akt phosphorylation and neuronal survival after traumatic brain injury in mice. Neurobiol Dis. 2002;9:294-304.

[18] Rebholz B, Kehe K, Ruzicka T, Rupec RA. Role of NF-kappaB/RelA and MAPK pathways in keratinocytes in response to sulfur mustard. J Invest Dermatol. 2008;128:1626-32. 
[19] Kaminska B. MAPK signalling pathways as molecular targets for anti-inflammatory therapy-from molecular mechanisms to therapeutic benefits. Biochim Biophys Acta. 2005;1754:253-62.

[20] Sharma GD, He J, Bazan HE. p38 and ERK1/2 coordinate cellular migration and proliferation in epithelial wound healing: evidence of cross-talk activation between MAP kinase cascades. J Biol Chem. 2003;278:21989-97.

[21] Seah CC, Phillips TJ, Howard CE, Panova IP, Hayes CM, Asandra AS, et al. Chronic wound fluid suppresses proliferation of dermal fibroblasts through a Ras-mediated signaling pathway. J Invest Dermatol. 2005;124:466-74.

[22] Ramet M, Lanot R, Zachary D, Manfruelli P. JNK signaling pathway is required for efficient wound healing in Drosophila. Dev Biol. 2002;241:145-56.

[23] Huh JE, Nam DW, Baek YH, Kang JW, Park DS, Choi DY, et al. Formononetin accelerates wound repair by the regulation of early growth response factor- 1 transcription factor through the phosphorylation of the ERK and p38 MAPK pathways. Int Immunopharmacol. 2011;11:46-54.

[24] Gustilo RB, Mendoza RM, Williams DN. Problems in the management of type III (severe) open fractures: a new classification of type III open fractures. J Trauma. 1984;24:742-6.

[25] Al-Mousawi AM, Kulp GA, Branski LK, Kraft R, Mecott GA, Williams FN, et al. Impact of anesthesia, analgesia, and euthanasia technique on the inflammatory cytokine profile in a rodent model of severe burn injury. Shock. 2010;34:261-8.

[26] Lindsey BA, Clovis NB, Smith ES, Salihu S, Hubbard DF. An animal model for open femur fracture and osteomyelitis--Part II: Immunomodulation with systemic IL-12. J Orthop Res. 2010;28:43-7. 
[27] Hulse RE, Kunkler PE, Fedynyshyn JP, Kraig RP. Optimization of multiplexed bead-based cytokine immunoassays for rat serum and brain tissue. J Neurosci Methods. 2004;136:87-98.

[28] Song G, Ouyang G, Bao S. The activation of Akt/PKB signaling pathway and cell survival. J Cell Mol Med. 2005;9:59-71.

[29] Mebratu Y, Tesfaigzi Y. How ERK1/2 activation controls cell proliferation and cell death: Is subcellular localization the answer? Cell Cycle. 2009;8:1168-75.

[30] Yeow K, Cabane C, Turchi L, Ponzio G, Derijard B. Increased MAPK signaling during in vitro muscle wounding. Biochem Biophys Res Commun. 2002;293:112-9.

[31] Keshavjee S, Zhang XM, Fischer S, Liu M. Ischemia reperfusion-induced dynamic changes of protein tyrosine phosphorylation during human lung transplantation. Transplantation. 2000;70:525-31.

[32] Irving EA, Bamford M. Role of mitogen- and stress-activated kinases in ischemic injury. J Cereb Blood Flow Metab. 2002;22:631-47.

[33] Tandara AA, Mustoe TA. Oxygen in wound healing--more than a nutrient. World J Surg. 2004;28:294-300.

[34] Sen CK. Wound healing essentials: let there be oxygen. Wound Repair Regen. 2009;17:1-18.

[35] Chiang B, Essick E, Ehringer W, Murphree S, Hauck MA, Li M, et al. Enhancing skin wound healing by direct delivery of intracellular adenosine triphosphate. Am J Surg. 2007;193:213-8.

[36] Jope RS, Johnson GV. The glamour and gloom of glycogen synthase kinase-3. Trends Biochem Sci. 2004;29:95-102. 
[37] Bassel-Duby R, Olson EN. Signaling pathways in skeletal muscle remodeling. Annu Rev Biochem. 2006;75:19-37.

[38] Guttridge DC. Signaling pathways weigh in on decisions to make or break skeletal muscle. Curr Opin Clin Nutr Metab Care. 2004;7:443-50.

[39] Fang CH, Li B, James JH, Yahya A, Kadeer N, Guo X, et al. GSK-3beta activity is increased in skeletal muscle after burn injury in rats. Am J Physiol Regul Integr Comp Physiol. 2007;293:R1545-51.

[40] Moore SF, van den Bosch MT, Hunter RW, Sakamoto K, Poole AW, Hers I. Dual regulation of glycogen synthase kinase 3 (GSK3)alpha/beta by protein kinase C (PKC)alpha and Akt promotes thrombin-mediated integrin alphaIIbbeta3 activation and granule secretion in platelets. J Biol Chem. 2013;288:3918-28.

[41] Park WY, Goodman RB, Steinberg KP, Ruzinski JT, Radella F, 2nd, Park DR, et al. Cytokine balance in the lungs of patients with acute respiratory distress syndrome. Am J Respir Crit Care Med. 2001;164:1896-903.

[42] Maier B, Lefering R, Lehnert M, Laurer HL, Steudel WI, Neugebauer EA, et al. Early versus late onset of multiple organ failure is associated with differing patterns of plasma cytokine biomarker expression and outcome after severe trauma. Shock. 2007;28:668-74.

[43] Simmons EM, Himmelfarb J, Sezer MT, Chertow GM, Mehta RL, Paganini EP, et al. Plasma cytokine levels predict mortality in patients with acute renal failure. Kidney Int. 2004;65:1357-65. 
[44] Cheon SS, Nadesan P, Poon R, Alman BA. Growth factors regulate beta-catenin-mediated TCF-dependent transcriptional activation in fibroblasts during the proliferative phase of wound healing. Experimental Cell Research. 2004;293:267-74.

[45] Cargnello M, Roux PP. Activation and function of the MAPKs and their substrates, the MAPK-activated protein kinases. Microbiol Mol Biol Rev. 2011;75:50-83.

[46] Aronson D, Wojtaszewski JF, Thorell A, Nygren J, Zangen D, Richter EA, et al. Extracellularregulated protein kinase cascades are activated in response to injury in human skeletal muscle. Am J Physiol. 1998;275:C555-61.

[47] Ishida Y, Kondo T, Takayasu T, Iwakura Y, Mukaida N. The essential involvement of crosstalk between IFN-gamma and TGF-beta in the skin wound-healing process. J Immunol. 2004;172:1848-55.

[48] Simon RA, Everhart JS, Nagaraja HN, Chaudhari AM. A case-control study of anterior cruciate ligament volume, tibial plateau slopes and intercondylar notch dimensions in ACL-injured knees. J Biomech. 2010;43:1702-7.

[49] Li Y, Ju L, Hou Z, Deng H, Zhang Z, Wang L, et al. Screening, verification, and optimization of biomarkers for early prediction of cardiotoxicity based on metabolomics. J Proteome Res. $2015 ; 14: 2437-45$.

[50] Longini M, Giglio S, Perrone S, Vivi A, Tassini M, Fanos V, et al. Proton nuclear magnetic resonance spectroscopy of urine samples in preterm asphyctic newborn: a metabolomic approach. Clin Chim Acta. 2015;444:250-6. 


\section{Chapter 3}

Using network centrality parameter analysis to investigate intraand extracellular signaling responses of a traumatic injury 


\section{Using network centrality parameter analysis to investigate intra- and extracellular signaling responses of a traumatic injury}

Investigations of cellular responses involved in injury and repair processes have generated valuable information contributing to the advancement of wound healing and treatments. Intra- and extracellular regulators of healing mechanisms, such as cytokines, signaling proteins, and growth factors, have been described to possess significant roles in facilitating optimal recovery. This study explored a collection of 30 spatiotemporal responses comprised of cytokines, proteins, phosphorylated proteins, and Caspase-3, measured in skeletal muscle tissue following a traumatic injury. To optimize the analysis of context specific data sets, a network centrality parameter approach was used to assess the impact of each response in relation to all other measured responses. This approach identified proteins that were substantially amplified and potentially central in the wound healing network by evaluation of their corresponding centrality parameter, radiality. Network analysis allowed us to distinguish the progression of healing that occurred at certain time points and regions of injury. Notably, inflammation and new tissue formation was proposed to occur by $168 \mathrm{~h}$ post-injury, while tissue 1 -cm away from the site of injury that experienced minor injury appeared to exhibit signs of new tissue formation as early as $24 \mathrm{~h}$ post-injury. In particular, hallmarks of inflammation, cytokines IL-1ß, IL-6, and IL-2 appear to have a pronounced impact at earlier time points ( $0-24 \mathrm{~h}$ post-injury), while intracellular proteins involved in cell proliferation, differentiation, or proteolysis (c-Jun, CREB, JNK, p38, p-c-Jun; p-MEK1, p-p38, p-STAT3) are more significant at later times (24-168 h). Overall, this study demonstrates the feasibility of a network analysis approach to extract significant information and also offers a spatiotemporal visualization of the intra- and extracellular signaling responses that regulate healing mechanisms. 


\subsection{Introduction}

The elucidation of molecular and signaling responses involved in traumatic injuries has gained considerable interest for potential treatments that may enhance the healing process. Previous studies have investigated the roles of cytokines, chemokines, growth factors [1], or signaling proteins [2] involved in the characteristic stages of wound repair: hemostasis, inflammation, tissue formation, and remodeling [3]. However, certain factors can influence the measured responses, such as varying classes and severities of injuries (e.g. fractures, minor cuts) and different tissue types (e.g. cutaneous tissue, bone, or myofibroblasts), which all appear to have distinct repair mechanisms [4]. Additionally, the elapsed time since the occurrence of the injury, as well as the spatial distribution of the zones of injury, may affect the measured response [5]. An increasing collection of intra- and extracellular regulators continues to emerge as prospective contributors of healing, allowing researchers to examine signaling pathways [6-7] or networks [8] involved in repair mechanisms. However, as the number of injury biomarkers grows, it can become difficult to interpret large datasets and translate the results into information useful to practitioners and researchers.

Complex datasets, particularly those describing biological responses, may benefit from a data-driven mathematical modelling or a network analysis approach [9]. Leading bioinformatic software specializing in network analysis include Ingenuity Pathway Analysis (IPA), Cytoscape, and Pathway Studio, to name a few. These software programs allow for the construction of molecular networks and signaling pathways, or the identification of involved molecular functions and even disease states using experimental data [10]. Network analyses of biochemical responses are best recognized as tools to evaluate protein-protein interactions, construct signal transduction 
pathways, or elucidate metabolic patterns in order to better understand how entire systems respond when perturbed by detrimental events [11]. Network analytics, such as centrality parameters, can also be informative in sorting through and dissecting large datasets. Centrality parameters describe how central one node (measured response) is, relative to all other nodes in the system. This method of analysis allows for an unbiased comparison of all observations regardless of biological hierarchal assignments (e.g. extracellular cytokines and intracellular phosphorylated proteins). Node centrality has been used to examine biological networks, such as protein-protein interactions resulting from chronic diseases [12] or phosphorylation responses elicited by toxic exposures [13]. In these studies, centrality parameters were used to identify biological components that were highly impactful, relative to all other measured responses in the network. A node with a high centrality can be interpreted as having a greater degree of likeness to all other nodes, while a node with a low centrality can be construed as having a more peripheral position in the network (behaving differently from all other nodes in the group) [14].

In this study, 30 intra and extracellular responses were measured in skeletal muscle tissue following a traumatic injury, in an effort to expand our understanding of the spatial and temporal signaling changes that occur during wound healing. Cytokine, total protein, phosphorylated protein, and Caspase-3 levels were observed at varying time points $(0,6,24,168 \mathrm{~h}$ post injury) and locations of injury (at the site of injury, 1-cm away). Eight different networks were constructed for each spatiotemporal condition (e.g. At the site of injury, $0 \mathrm{~h}$ ) and the centrality parameter, radiality, was obtained for each node. Relative radiality values identified nodes that had particularly distinctive responses, potentially holding a prominent role in driving the mechanisms of wound healing. The dataset was also examined with IPA to provide a comparison between a recognized 
software application, often used for analyzing and integrating large biological datasets, and the proposed method.

\subsection{Materials and methods}

\subsubsection{Data description}

Intra and extracellular biomolecule concentrations were measured after a severe fracture at the site of the injury, 1-cm away, and in the uninjured leg of adult male Sprague-Dawley rats (as described in Chapter 2, Currie, et al. [5], and Han, et al. [15]. In addition to examining the spatial differences of the injury, molecular responses were also measured at $0 \mathrm{~h}, 6 \mathrm{~h}, 24 \mathrm{~h}$, and $168 \mathrm{~h}$ postinjury. A total of 30 targets were selected, including nine proteins, 14 phosphoproteins, six cytokines, and one protease (listed in Table 3.1). Briefly, the relative abundance of total protein and phosphoprotein were measured with a Bio-plex kit containing polystyrene, non-magnetic antibody coated beads. Cytokine responses were measured with a Bio-Plex Pro multiplexed magnetic bead-based immunoassay reagent kit and Caspase-3 activity was measured with a fluorescence assay kit from Cayman Chemical (Item No. 10009135). All procedures were reviewed and approved by the West Virginia University Animal Care and Use Committee.

\subsubsection{Data processing}

Data were analyzed using Prism 5 (GraphPad, San Diego, CA), and SAS JMP Pro 12.0.1 (Carey, NC). Total protein, phosphoprotein, cytokine, and Caspase-3 abundances measured in muscle tissue at the site of fracture and 1-cm away from the site were normalized to levels found in tissue collected from the uninjured leg. Normalizing to the uninjured leg served as a systemic 
control, accounting for any responses that were not directly involved in the response to the local injury. Additionally, normalization accounted for any discrepancies brought on by the differences in measurement units (e.g. large RFU signals of phosphorylation levels vs. ng/g of cytokine response) in the calculation of radiality.

\subsubsection{Network evaluation with Ingenuity Pathway Analysis}

The relative responses were also investigated with QIAGEN's Ingenuity® Pathway Analysis (IPA®, QIAGEN Redwood City). The 30 nodes were split into two groups to allow for analysis: one group consisted of total protein, cytokine, and Caspase-3 responses, and the second group included only relative phosphorylated protein responses. Proposed signaling networks for all time-points $(0,6,24,168 \mathrm{~h})$ and locations (at fracture, 1-cm away) of injury were generated. All networks are comprised of nodes from the experimental dataset, as well as other projected nodes likely to be involved in the network, based on the Ingenuity Knowledge Base. Up- and downregulated responses are represented by red and green color coding, respectively. IPA additionally reported potential molecular and cellular functions describing the network, along with the corresponding scores (negative p-value of Fisher's exact test). The Fisher's exact test (p-value) describes the probability that the associations between the experimental nodes and molecular networks are due to random chance. 
Table 3.1.

Intra and Extracellular biomolecules measured after traumatic injury

\begin{tabular}{|c|c|}
\hline & Target \\
\hline \multirow{9}{*}{ Protein } & Akt \\
\hline & c-Jun \\
\hline & CREB \\
\hline & ERK1/2 \\
\hline & JNK \\
\hline & MEK1 \\
\hline & p38 \\
\hline & p53 \\
\hline & p90RSK \\
\hline \multirow{14}{*}{$\begin{array}{l}\text { Phosphorylated protein } \\
\text { (site) }\end{array}$} & p-Akt (Ser473) \\
\hline & p-c-Jun (Ser63) \\
\hline & p-CREB (Ser133) \\
\hline & p-ERK1/2 (Thr202/Tyr204, Thr185/Tyr187) \\
\hline & p-GSK-3 $\alpha / ß$ (Ser21/Ser9) \\
\hline & p-HSP27 (Ser78) \\
\hline & $\mathrm{p}-\mathrm{I} \kappa \mathrm{B} \alpha(\operatorname{Ser} 536)$ \\
\hline & p-JNK (Thr183/Tyr185) \\
\hline & p-MEK1 (Ser217/Ser221) \\
\hline & p-p38 (Thr180/Tyr182) \\
\hline & p-p70S6K (Thr421/Ser424) \\
\hline & p-p90RSK (Ser380) \\
\hline & p-STAT2 (Tyr689) \\
\hline & p-STAT3 (Tyr705) \\
\hline \multirow{6}{*}{ Cytokine } & IL-1 $\alpha$ \\
\hline & IL-1ß \\
\hline & IL-2 \\
\hline & IL-6 \\
\hline & TNF- $\alpha$ \\
\hline & MIP-1 $\alpha$ \\
\hline Protease & Caspase-3 \\
\hline
\end{tabular}




\subsubsection{Network centrality parameter analysis}

Using SAS JMP, Euclidean distances between each pair of normalized observation (total protein, phosphoprotein, cytokine, Caspase-3) were determined for all time points and locations of injury (e. g. Time $=0 \mathrm{~h} / \mathrm{At}$ the site of fracture). Euclidean distance is defined as:

$$
E(v, \omega)=\sqrt{\sum_{i=1}^{n}\left(\left(\text { normalized response } v_{i}\right)-\left(\text { normalized response } \omega_{i}\right)\right)^{2}}
$$

where $v$ and $\omega$ represent the 2 responses for which the distance between is being calculated, and $\mathrm{n}$ signifies the replicate number.

To construct a spatiotemporal network of the measured responses to the injury, Euclidean distances between each pair of normalized observation (node) were used to calculate the node centrality parameter, radiality:

\section{Radiality -}

$$
C_{\operatorname{rad}(v)}=\frac{\sum_{\omega \in N}(\Delta G+1-\operatorname{dist}(v, \omega))}{n-1}
$$

where $\Delta \mathrm{G}$ represents the network $(\mathrm{N})$ diameter (longest path length connecting any 2 nodes), dist $(v, \omega)$ is defined as the shortest path between two nodes $(v, \omega)$, and $n$ is the number of nodes in the network. Briefly, radiality describes how close (high values) or distant (low values) in proximity one node is from all of the other nodes in the network. High radiality values will result 
if the distances are short, while low values will be produced by long distances. Radiality values were normalized to the average radiality for all proteins in the network, indicating that proteins with relative values higher than 1 are more central than average, and proteins with relative values lower than 1 are less central than average. A threshold of the average radiality \pm the standard deviation was set to identify nodes with significant radiality outcomes.

\subsection{Results}

\subsubsection{Relative spatial and temporal cytokine response}

Responses of the following six cytokines were normalized to levels detected in the uninjured leg: IL-6, IL-1ß, IL-2, TNF- $\alpha$, IL-1 $\alpha$, and MIP-1 $\alpha$ (Figure 3.1). IL-6 and IL-1ß levels were highest at $6 \mathrm{~h}$ in tissue from the site of fracture, with a significantly higher $(p<0.05)$ response at the site of fracture compared to samples acquired from 1-cm away. Tissue located 1-cm away experienced a considerable increase in IL- 2 concentration at $24 \mathrm{~h}$ post fracture, while MIP- $1 \alpha$ concentrations at the site of fracture slightly decreased $24 \mathrm{~h}$ following the injury. IL-1 $\alpha$ and TNF$\alpha$ did not exhibit any statistically significant spatial nor temporal differences.

\subsubsection{Relative spatial and temporal protein response}

Total protein concentrations were normalized to levels measured in the uninjured tissue for the following 9 targets: c-Jun, CREB, p38, ERK1/2, JNK, Akt, MEK1, p53, and p90RSK (Figure 3.2). The relative protein levels of c-Jun, CREB, p38, ERK1/2, JNK, and Akt at the site of the fracture -168 h post fracture were significantly $(p<0.05)$ increased, compared to responses measured at 0,6 and $24 \mathrm{~h}$. These protein levels were also significantly higher at the site of injury 
than in the uninjured leg $(>1)$ at $168 \mathrm{~h}$. There were no significant temporal or spatial differences for those 6 proteins at the three earlier times $(0,6$, and $24 \mathrm{~h})$. In tissue 1-cm away from the fracture, only JNK and p38 responses were statistically increased at $168 \mathrm{~h}$, in comparison to levels found at 0, 6, and $24 \mathrm{~h}$. Furthermore, JNK and p38 levels at time point $168 \mathrm{~h}$ were significantly higher in tissue 1-cm away from the fractured location when compared to tissue from the uninjured leg. Notable spatial differences of c-Jun and CREB responses were also observed between the two regions of injury, with significantly higher levels measured at the site of fracture for both proteins. Additionally, MEK1 protein concentration at the site of fracture decreased considerably at $24 \mathrm{~h}$ (statistically different from MEK1 response in uninjured tissue). There were no spatial nor temporal differences for p53 and p90RSK and all measurements were statistically comparable to levels measured in the uninjured tissue. 
IL-6
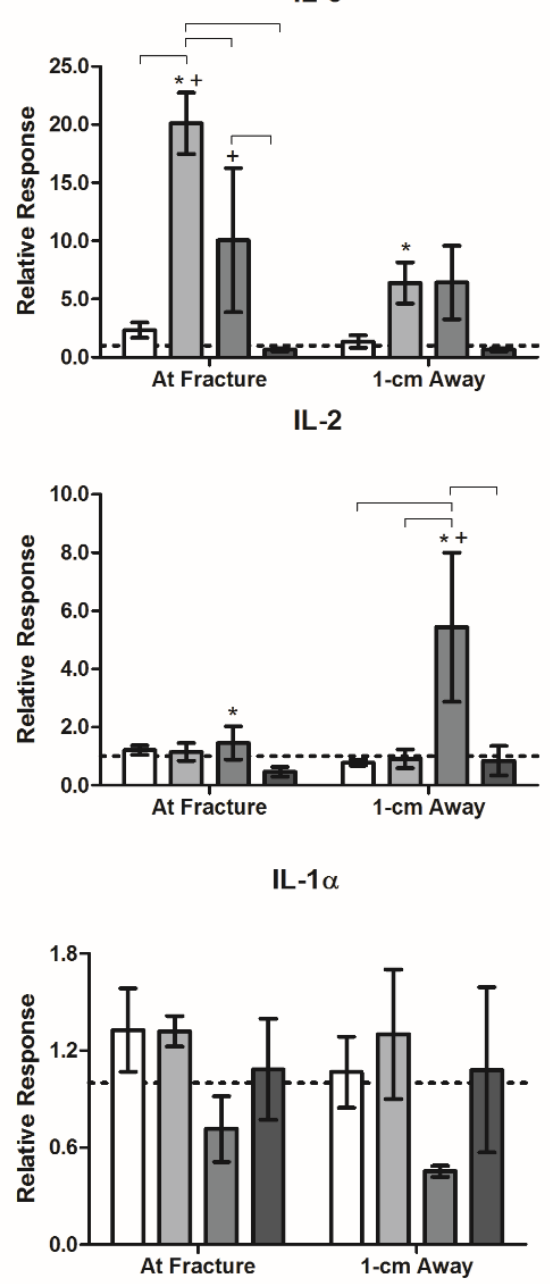

IL-1 $\beta$
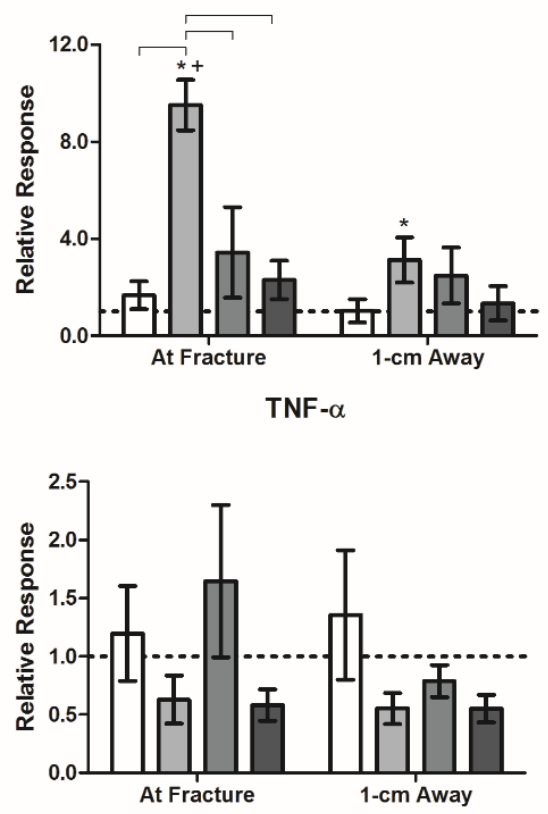

MIP-1 $\alpha$

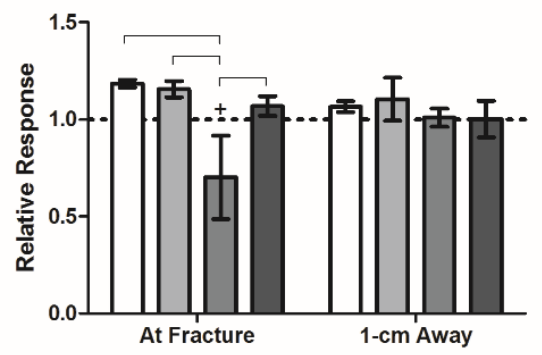

Figure 3.1. Relative cytokine levels measured across time and injury location in muscle tissue resulting from a femur fracture. Relative cytokine response (normalized to levels measured in uninjured leg) were observed across four time points and different regions of injury following the femur fracture: IL-1 $\alpha$, IL-1 1 , IL-2, IL-6, TNF- $\alpha$, and MIP- $1 \alpha$. Statistically significant $(p<0.05)$ differences were determined by 2-way ANOVA with a Bonferroni post-test. Temporal differences are notated with brackets pairing certain time points when substantial alterations in protein response were observed. Statistically significant spatial differences are marked with a matching symbol $(*)$. Responses at the different regions of injury (at the site of fracture and 1-cm away) that were significantly higher $(>1)$ or lower $(<1)$ than levels found in the uninjured leg are indicated with the + symbol. Error bars reflect \pm standard error of the mean. 

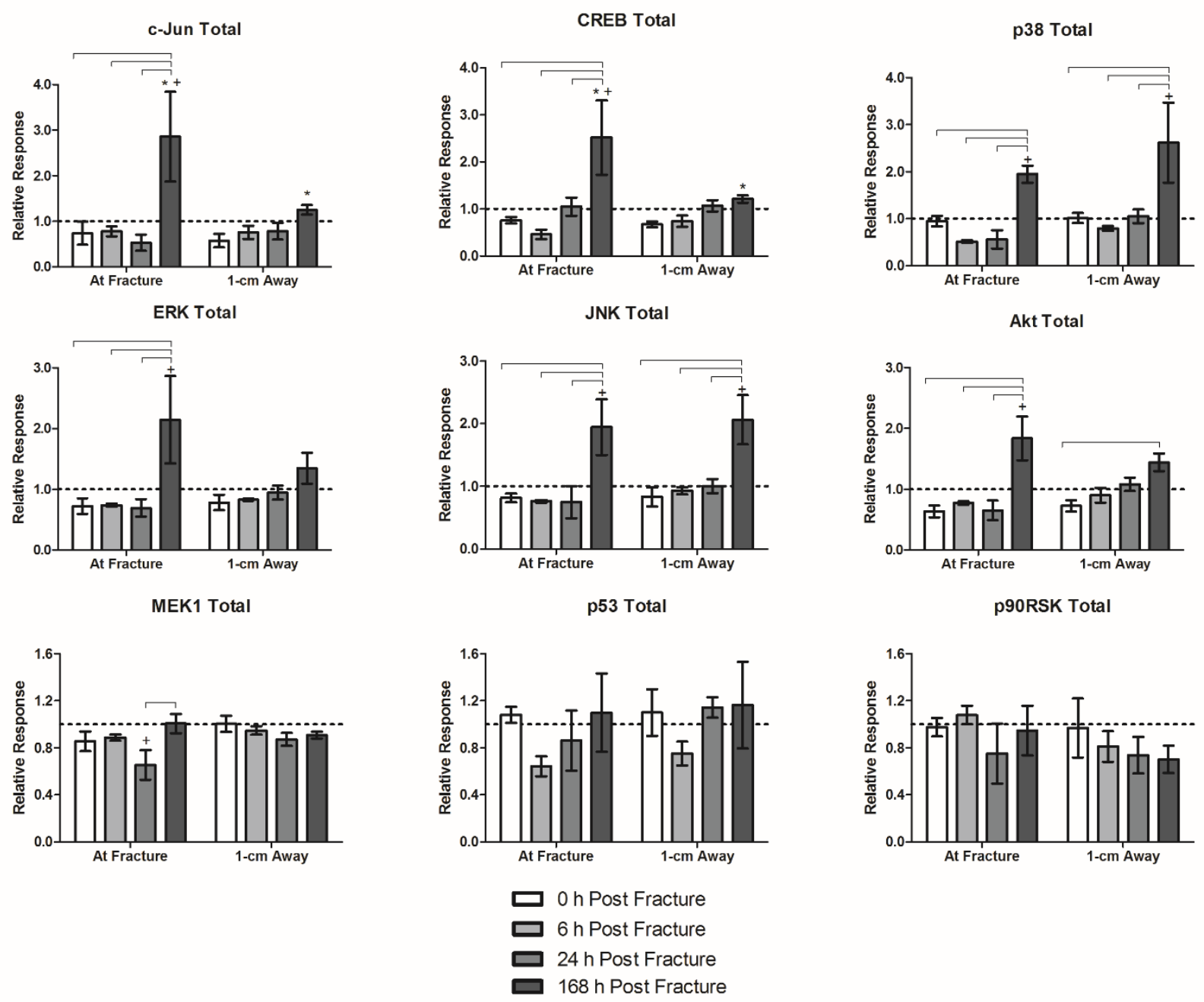

Figure 3.2. Relative protein response measured across time and injury location in muscle tissue resulting from a femur fracture. Relative response (normalized to levels measured in uninjured leg) associated with total protein concentrations of the following proteins were observed across four time points and different regions of injury following the femur fracture: Akt, c-Jun, CREB, ERK1/2, JNK, MEK1, p38, p53, and p90RSK. Statistically significant $(p<0.05)$ differences were determined by 2-way ANOVA with a Bonferroni post-test. Temporal differences are notated with brackets pairing certain time points when substantial alterations in protein response were observed. Statistically significant spatial differences are marked with a matching symbol (*). Responses at the different regions of injury (at the site of fracture and 1-cm away) that were significantly higher $(>1)$ or lower $(<1)$ than levels found in the uninjured leg are indicated with the + symbol. Error bars reflect \pm standard error of the mean. 


\subsubsection{Relative spatial and temporal response of phosphorylated proteins}

To further examine the impact of the observed proteins (c-Jun, CREB, p38, ERK1/2, JNK, Akt, MEK1, p53, and p90RSK), associated phosphorylation levels were also measured. Phosphoprotein responses of 6 additional targets were added as well: STAT3, p70S6K, STAT2, HSP27, I $\kappa B \alpha$, GSK-3 $\alpha / \beta$ (Figures 3.3 and 3.4). At the site of fracture, spatiotemporal variations in the phosphorylation of STAT3, c-Jun, p70S6K, and Akt were observed: significantly $(p<0.05)$ increased responses at $24 \mathrm{~h}$ for STAT3, increased response at $168 \mathrm{~h}$ for c-Jun and p70S6K, and decreased response at $6 \mathrm{~h}$ and $24 \mathrm{~h}$ for Akt. In tissue 1-cm away from the fracture, phosphorylation on MEK1, STAT3, ERK1/2, p38, p70S6K, CREB and GSK-3 $\alpha / \beta$ proteins displayed significant changes over time. Significantly elevated responses at $24 \mathrm{~h}$ were measured for MEK1, STAT3, ERK1/2, p70S6K, CREB, and at $168 \mathrm{~h}$ for p38. A reduction in GSK-3 $\alpha / \beta$ phosphorylation occurred at $0 \mathrm{~h}$ and increased to comparable levels found in the uninjured leg at the later time points for 1-cm away.

Considerable spatial differences were also observed between the site of fracture and $1-\mathrm{cm}$ away for MEK1, c-Jun, and p70S6K: phosphorylation responses in tissue at the site of fracture were significantly higher for c-Jun and p70S6K at $168 \mathrm{~h}$, while the response was higher in 1-cm away tissue at $24 \mathrm{~h}$ for MEK1. Although there were no significant differences over time or between the two sampling locations for the phosphorylation of JNK and STAT2, there was a slight increase in response at $6 \mathrm{~h}$ at the site of fracture, where the response was higher than levels detected in the uninjured leg (relative response is $>1$ ). Phosphorylation of HSP27, I $\mathrm{KB} \alpha$, and p90RSK remained constant over time for the two regions associated with the injury, and were not significantly different than responses observed in the uninjured leg. 
p-MEK1
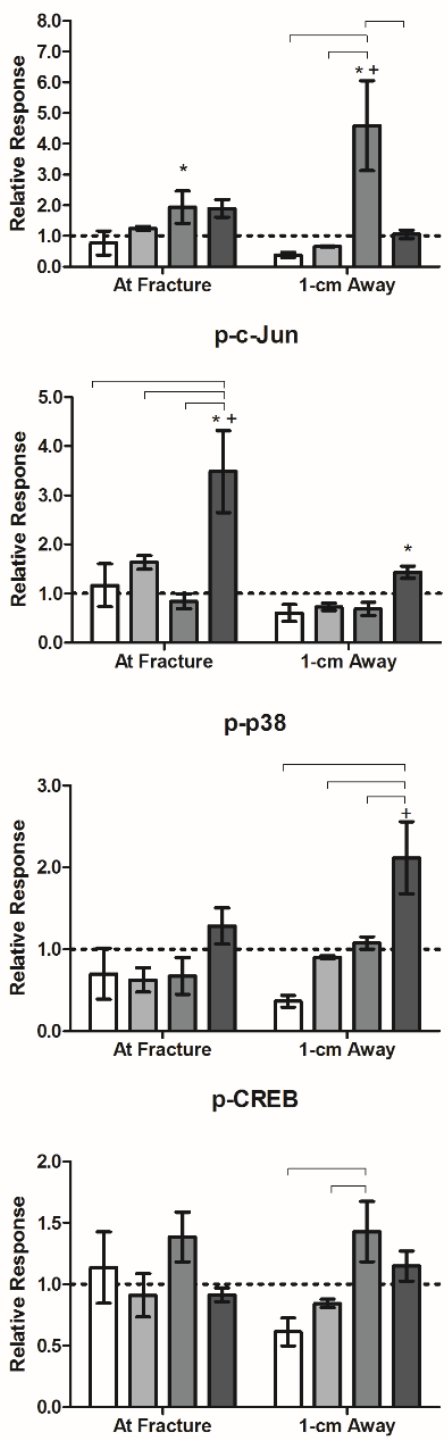

p-STAT3

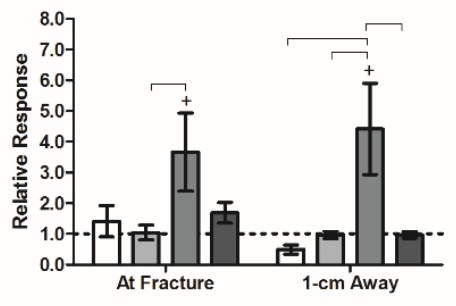

p-ERK1/2

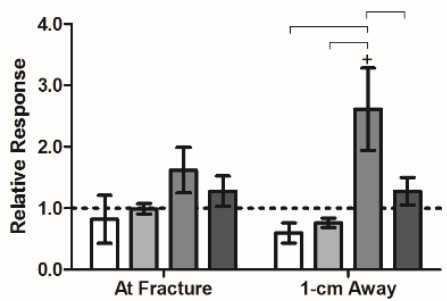

p-p70S6K

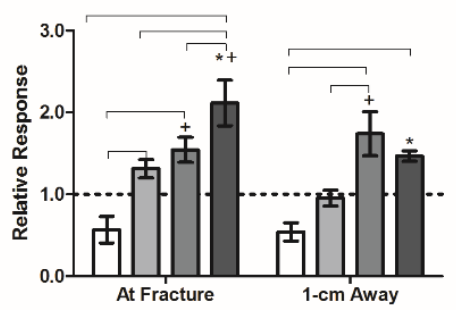

$\square 0$ h Post Fracture

$\square 6$ h Post Fracture

$\square 24$ h Post Fracture

$\square 168 \mathrm{~h}$ Post Fracture

Figure 3.3. Relative phosphorylation response measured across time and injury location in muscle tissue resulting from a femur fracture. Relative phosphorylation response (normalized to levels measured in uninjured leg) of the following proteins were observed across four time points and different regions of injury following the femur fracture: Akt, c-Jun, CREB, ERK1/2, GSK-3 $\alpha / \beta$, HSP27, IкB $\alpha$. Statistically significant $(p<0.05)$ differences were determined by 2 -way ANOVA with a Bonferroni post-test. Temporal differences are notated with brackets pairing certain time points when substantial alterations in protein response were observed. Statistically significant spatial differences are marked with a matching symbol $(*)$. Responses at the different regions of injury (at the site of fracture and 1-cm away) that were significantly higher $(>1)$ or lower $(<1)$ than levels found in the uninjured leg are indicated with the + symbol. Error bars reflect \pm standard error of the mean. 

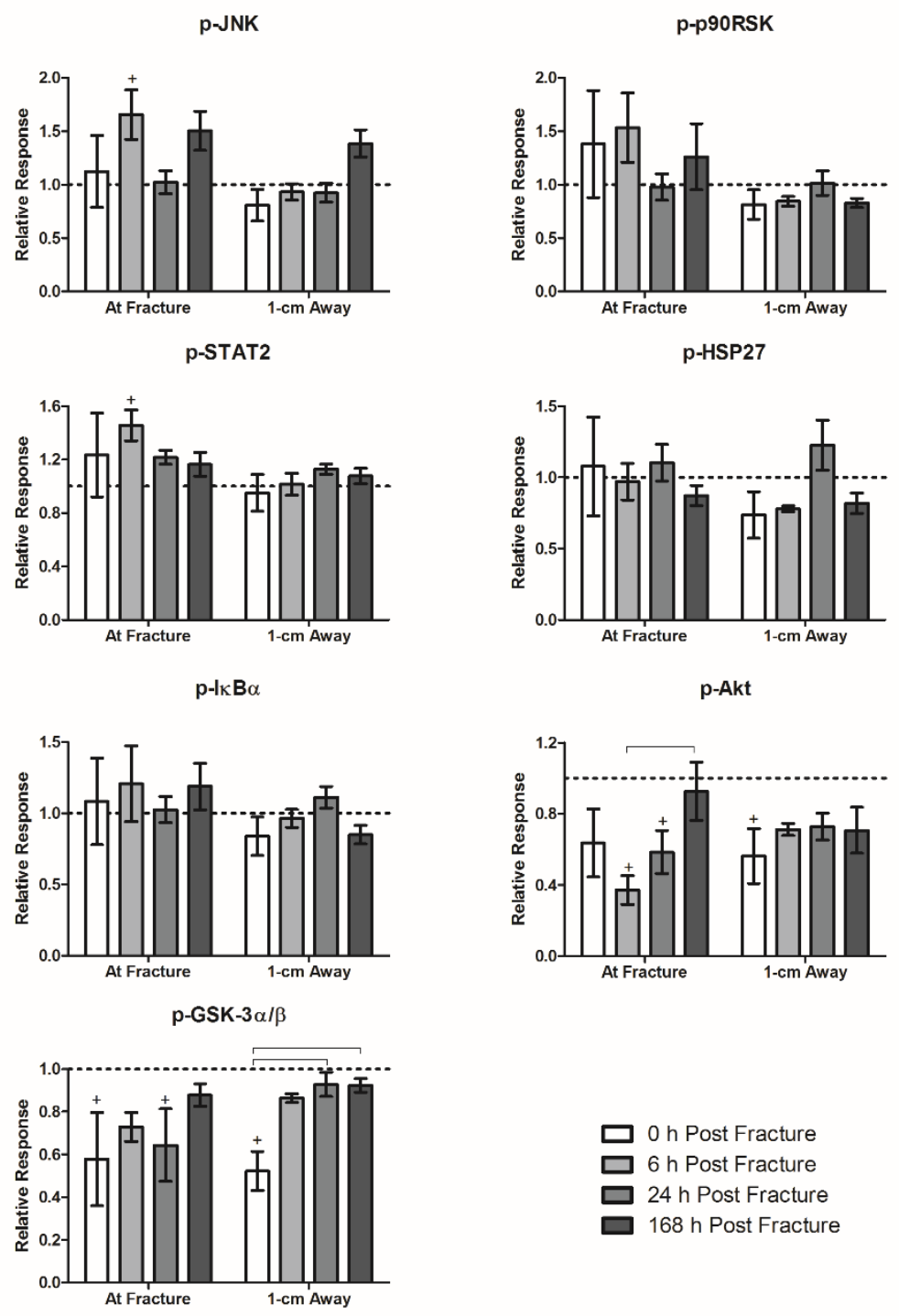

Figure 3.4. Relative phosphorylation response measured across time and injury location in muscle tissue resulting from a femur fracture. Relative phosphorylation response (normalized to levels measured in uninjured leg) of the following proteins were observed across four time points and different regions of injury following the femur fracture: JNK, MEK1, p38, p70S6K, p90RSK, STAT2, and STAT3. Statistically significant $(p<0.05)$ differences were determined by 2 -way ANOVA with a Bonferroni post-test. Temporal differences are notated with brackets pairing certain time points when substantial alterations in protein response were observed. Statistically significant spatial differences are marked with a matching symbol (*). Responses at the different regions of injury (at the site of fracture and 1-cm away) that were significantly higher (>1) or lower $(<1)$ than levels found in the uninjured leg are indicated with the + symbol. Error bars reflect \pm standard error of the mean. 


\subsubsection{Relative spatial and temporal caspase-3 response}

The activity of Caspase-3 at the site of the fracture and 1-cm away was measured and normalized to levels found in the uninjured leg (Figure 3.5). At the site of the fracture, Caspase-3 activity was initially reduced $(0$ and $24 \mathrm{~h})$ and then increased at $168 \mathrm{~h}$, in relation to the uninjured leg. In tissue 1-cm away from the fracture, relative responses observed at 24 and $168 \mathrm{~h}$ were significantly different from each other. Additionally, at $0 \mathrm{~h}$, activity was substantially decreased at the fracture compared to the region 1-cm away.

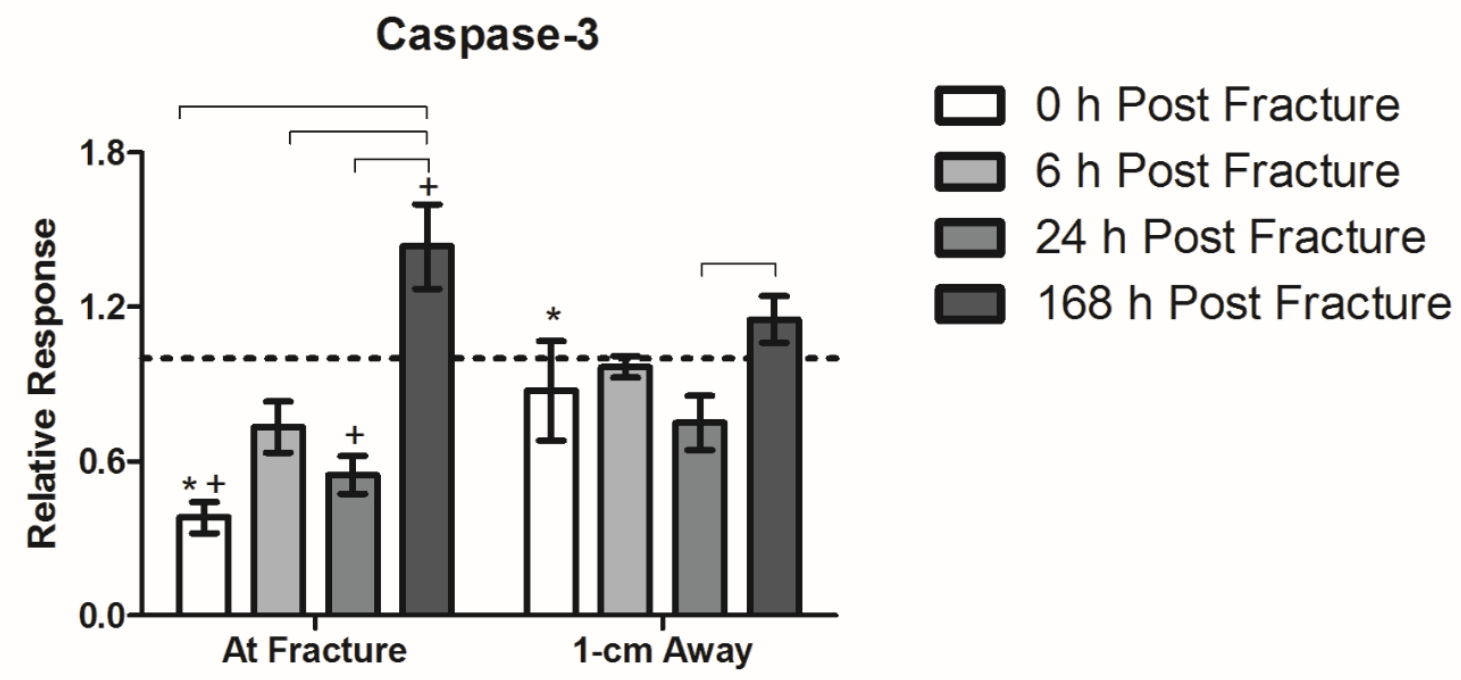

Figure 3.5. Relative Caspase-3 activity measured across time and injury location in muscle tissue resulting from a femur fracture. Relative Caspase-3 response (normalized to levels measured in uninjured leg) were observed across four time points and different regions of injury following the femur fracture. Statistically significant $(p<0.05)$ differences were determined by 2-way ANOVA with a Bonferroni post-test. Temporal differences are notated with brackets pairing certain time points when substantial alterations in protein response were observed. Statistically significant spatial differences are marked with a matching symbol $(*)$. Responses at the different regions of injury (at the site of fracture and 1-cm away) that were significantly higher $(>1)$ or lower $(<1)$ than levels found in the uninjured leg are indicated with the + symbol. Error bars reflect \pm standard error of the mean. 


\subsubsection{IPA generated networks}

Since all networks were constructed from the same collection of nodes, the overall components of the networks and the general connectivity of the nodes were similar across all time points and locations of injury. The only major difference shown among the networks are the upregulated and downregulated responses that did vary temporally and spatially. Two examples of IPA generated networks using the protein, cytokine, and protease or the phosphoprotein dataset are shown (Figures 3.6 and 3.7). Temporal changes between 0 and $168 \mathrm{~h}$ post-injury were demonstrated with the protein, cytokine, and protease network, while spatial differences between the two injury regions, at $6 \mathrm{~h}$ post-injury, were observed with the phosphoprotein network. The generated networks using protein, cytokine, and protease responses were postulated to encompass cellular processes including free radical scavenging, cell death and survival, skeletal and muscular disorders, cell signaling, small molecule biochemistry, drug metabolism, and cardiac necrosis/cell death. Cellular processes suggested to be involved in the phosphoprotein networks include cell death and survival, cell morphology, cellular development, lipid metabolism, small molecule biochemistry, organismal injury and abnormalities, cellular movement, skeletal and muscular disorders, and free radical scavenging (Table 3.2). 

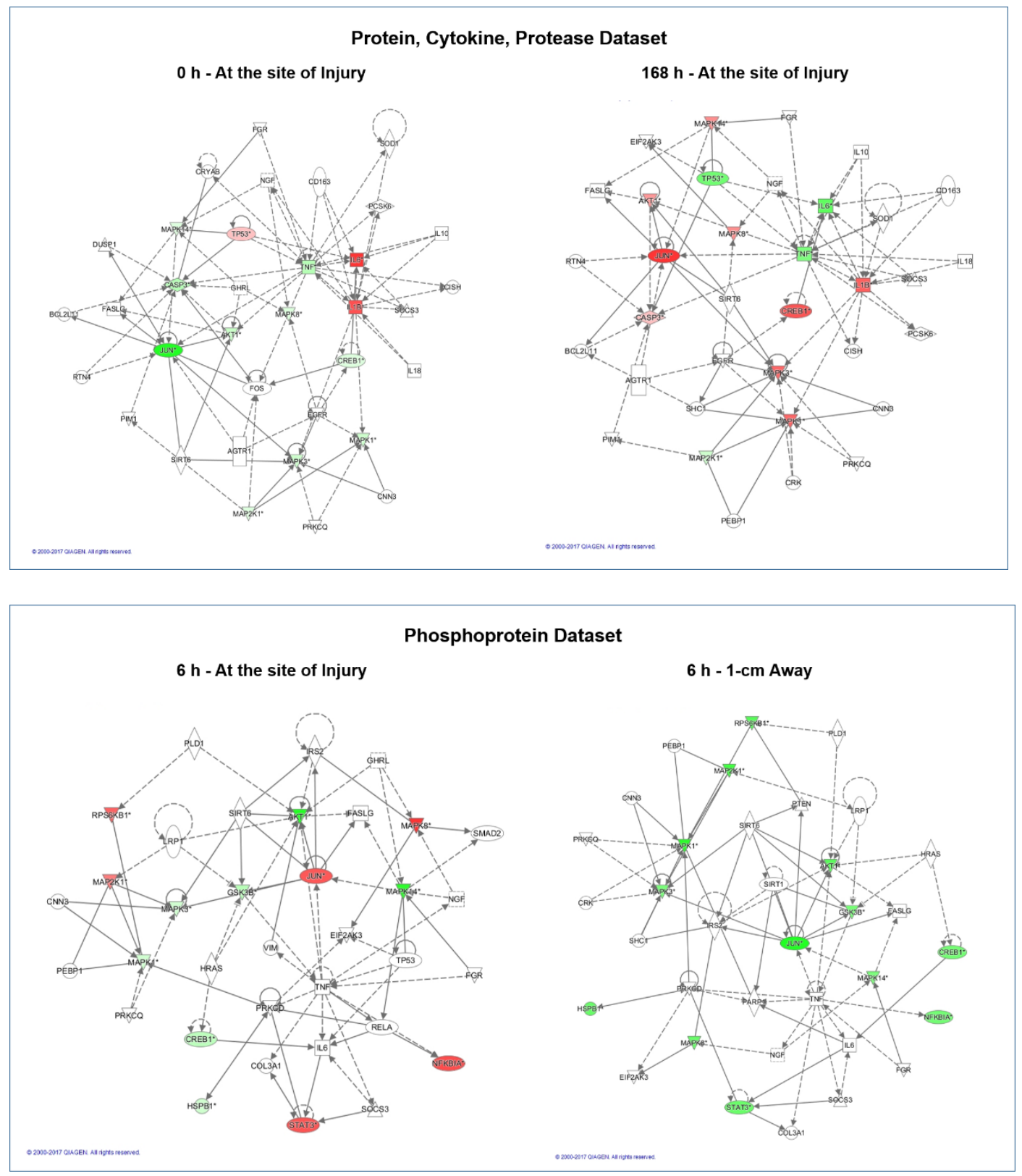

Figures 3.6 (top) and 3.7 (bottom). IPA generated networks constructed from protein, cytokine, phosphoprotein, and protease dataset. Proposed signaling networks generated by IPA used experimental relative protein, cytokine and protease responses at the site of injury for $0 \mathrm{~h}$ and 168 $\mathrm{h}$ post-injury, depicting the similarities and differences between disparate temporal networks (top). Networks portraying phosphoprotein responses at $6 \mathrm{~h}$ for both injury locations (at the site and 1$\mathrm{cm}$ away) are shown, illustrating the similarities and differences between disparate spatial networks (bottom). All networks were supplemented with other projected nodes likely to be involved in the network, based on the Ingenuity Knowledge Base. Up- and downregulated responses are represented by red and green color coding, respectively. 
Table 3.2.

Top IPA Networks

\begin{tabular}{|c|c|c|c|c|c|c|c|c|}
\hline \multirow{2}{*}{$\begin{array}{r}\text { Time } \\
\text { post-injury (h) }\end{array}$} & \multicolumn{4}{|c|}{ At the site of injury } & \multicolumn{4}{|c|}{$\underline{1-c m}$ away from injury } \\
\hline & $\begin{array}{l}\text { IPA Network (Protein } \\
\text { Cytokine, Caspase-3) }\end{array}$ & Score & $\begin{array}{c}\text { IPA Network } \\
\text { (Phosphorylated Protein) }\end{array}$ & Score & $\begin{array}{l}\text { IPA Network (Protein } \\
\text { Cytokine, Caspase-3) }\end{array}$ & Score & $\begin{array}{l}\text { IPA Network } \\
\text { (Phosphorylated } \\
\text { Protein) }\end{array}$ & Score \\
\hline $\mathbf{0}$ & $\begin{array}{c}\text { Free radical } \\
\text { scavenging, Cell death } \\
\text { and survival, Skeletal } \\
\text { and muscular } \\
\text { disorders }\end{array}$ & 22 & $\begin{array}{l}\text { Cell death and } \\
\text { survival, Cell } \\
\text { morphology, Cellular } \\
\text { development }\end{array}$ & 22 & $\begin{array}{l}\text { Cell death and survival, } \\
\text { Skeletal and muscular } \\
\text { disorders, Free radical } \\
\text { scavenging }\end{array}$ & 22 & $\begin{array}{l}\text { Cell death and } \\
\text { survival, Cell } \\
\text { morphology, } \\
\text { Cellular } \\
\text { development }\end{array}$ & 22 \\
\hline 6 & $\begin{array}{l}\text { Cell signaling, Small } \\
\text { molecule } \\
\text { biochemistry, drug } \\
\text { metabolism }\end{array}$ & 22 & $\begin{array}{c}\text { Cell death and } \\
\text { survival, Lipid } \\
\text { metabolism, Small } \\
\text { molecule biochemistry }\end{array}$ & 22 & $\begin{array}{l}\text { Cell signaling, Small } \\
\text { molecule biochemistry, } \\
\text { Drug metabolism }\end{array}$ & 22 & $\begin{array}{l}\text { Cell death and } \\
\text { survival, Skeletal } \\
\text { and muscular } \\
\text { disorders, Cardiac } \\
\text { hypertrophy }\end{array}$ & 22 \\
\hline 24 & $\begin{array}{c}\text { Cell death and } \\
\text { survival, Skeletal and } \\
\text { muscular disorders, } \\
\text { Cardiac necrosis/cell } \\
\text { death }\end{array}$ & 22 & $\begin{array}{c}\text { Cell death and } \\
\text { survival, Organismal } \\
\text { injury and abnormalities, } \\
\text { Cellular movement }\end{array}$ & 22 & $\begin{array}{l}\text { Cell death and survival, } \\
\text { Skeletal and muscular } \\
\text { disorders, Cardiac } \\
\text { necrosis/cell death }\end{array}$ & 22 & $\begin{array}{c}\text { Cell death and } \\
\text { survival, , Cardiac } \\
\text { hypertrophy, } \\
\text { Cardiovascular } \\
\text { disease }\end{array}$ & 22 \\
\hline 168 & $\begin{array}{c}\text { Free radical } \\
\text { scavenging, Cell death } \\
\text { and survival, Skeletal } \\
\text { and muscular } \\
\text { disorders }\end{array}$ & 22 & $\begin{array}{l}\text { Cell death and } \\
\text { survival, Skeletal and } \\
\text { muscular disorders, Free } \\
\text { radical scavenging }\end{array}$ & 22 & $\begin{array}{l}\text { Free radical scavenging, } \\
\text { Cell signaling, Small } \\
\text { molecule biochemistry }\end{array}$ & 22 & $\begin{array}{l}\text { Cell death and } \\
\text { survival, Skeletal } \\
\text { and muscular } \\
\text { disorders, Cell } \\
\text { morphology }\end{array}$ & 22 \\
\hline
\end{tabular}

Network scores: negative p-value of Fisher's exact test 


\subsubsection{Normalized radiality of all 30 nodes}

Radiality values that were determined for each node (response) were normalized to the average radiality value for each dataset (e.g. At Fracture, $0 \mathrm{~h}$ ), as presented in Tables 3.3 and 3.4. Nodes with significant radiality outcomes (significance threshold: average radility \pm standard deviation) are bolded. Normalization to the average was performed to allow for cross comparision across time and location. Monitoring the changes in radiality for each node over time, or identifying when the value significantly rises or falls, can identify critical times when a certain node behaves similiarly to or deviates from the rest of the nodes in the network. At the site of the fracture, normalized radiality of most nodes were comparable to the average $(=1)$ and remained unchanged over all 4 time points: Akt, ERK1/2, JNK, MEK1, p38, p53, p90RSK, p-Akt, p-CREB, p-ERK1/2, p-GSK-3 $\alpha / 3$, p-HSP27, p-IкB $\alpha$, p-JNK, p-MEK1, p-p38, p-p70S6K, p-p90RSK, pSTAT2, p-STAT3, IL-1 $\alpha$, IL-2, MIP-1 $\alpha$, TNF- $\alpha$, Caspase-3. Figure 3.8 shows normalized radiality scores for certain nodes (c-Jun, CREB, p-c-Jun) decreased considerably only at $168 \mathrm{~h}$, while IL$1 ß$ and IL-6 responses contributed to significantly low radiality scores at $0,6,168 \mathrm{~h}$, and $0,6,24$ $\mathrm{h}$, respectively.

In tissue 1-cm away from the fracture, the associated normalized radiality of most nodes were also comparable to the average ( $=1$ ) for all 4 time points: Akt, c-Jun, CREB, ERK1/2, MEK1,

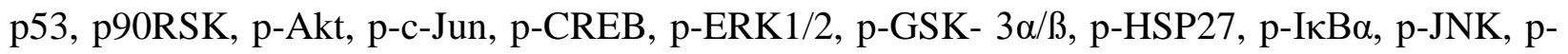
p70S6K, p-p90RSK, p-STAT2, IL-1 $\alpha$, MIP-1 $\alpha$, and Caspase-3. Figure 3.9 shows radiality for TNF- $\alpha$ was notably reduced at $0 \mathrm{~h}$, but increased to a value $\sim 1$ at all later time points. Only at 24 h, radiality scores of p-MEK1, p-STAT3, and IL-2 were significantly decreased. In addition, radiality scores for JNK, p38, and p-p38 were $\sim 1$ at the earlier time points $(0-24 \mathrm{~h})$, but dropped 
to lower scores at $168 \mathrm{~h}$. Radiality values for IL-1ß and IL-6 were significantly low at $0,6 \mathrm{~h}$, and $0,6,24 \mathrm{~h}$, respectively, which is similar to radiality determined for responses measured at the site of fracture.

Table 3.3

Normalized radiality of nodes at the site of injury

\begin{tabular}{ccccc}
\hline Node & $\mathbf{0 ~ h}$ & $\mathbf{6 ~ h}$ & $\mathbf{2 4} \mathbf{h}$ & $\mathbf{~}$ \\
\hline Akt Total & 1.057 & 1.049 & 1.052 & 1.046 \\
c-Jun Total & 1.074 & 1.048 & 1.046 & $\mathbf{0 . 6 0 1}$ \\
CREB Total & 1.060 & 1.038 & 1.053 & $\mathbf{0 . 7 7 5}$ \\
ERK1/2 Total & 1.079 & 1.048 & 1.052 & 0.862 \\
JNK Total & 1.069 & 1.048 & 1.046 & 1.010 \\
MEK1 Total & 0.999 & 1.050 & 1.052 & 1.120 \\
p38 Total & 0.977 & 1.041 & 1.046 & 0.944 \\
p53 Total & 1.061 & 1.044 & 1.050 & 1.083 \\
p9RSK Total & 1.067 & 1.049 & 1.047 & 1.096 \\
p-Akt & 1.060 & 1.035 & 1.050 & 1.084 \\
p-c-Jun & 1.005 & 1.036 & 1.049 & $\mathbf{0 . 5 2 8}$ \\
p-CREB & 1.066 & 1.047 & 1.042 & 1.101 \\
p-ERK1/2 & 1.019 & 1.046 & 1.029 & 1.002 \\
p-GSK-3 $\alpha / \beta$ & 1.038 & 1.048 & 1.051 & 1.076 \\
p-HSP27 & 1.062 & 1.051 & 1.048 & 1.089 \\
p-IKB $\alpha$ & 1.084 & 1.042 & 1.045 & 1.102 \\
p-JNK & 1.060 & 1.030 & 1.053 & 1.033 \\
p-MEK1 & 0.997 & 1.046 & 1.005 & 1.020 \\
p-p38 & 1.040 & 1.042 & 1.046 & 1.028 \\
p-p70SK6 & 1.048 & 1.046 & 1.040 & 0.944 \\
p-p90RSK & 0.936 & 1.036 & 1.049 & 1.048 \\
p-STAT2 & 1.056 & 1.042 & 1.044 & 1.101 \\
p-STAT3 & 0.915 & 1.042 & 0.880 & 1.062 \\
IL-1 $\alpha$ & 0.952 & 1.044 & 1.047 & 1.113 \\
IL-1ß & $\mathbf{0 . 7 1 7}$ & $\mathbf{0 . 6 6 4}$ & 0.829 & $\mathbf{0 . 8 3 8}$ \\
IL-2 & 1.072 & 1.044 & 1.037 & 0.997 \\
IL-6 & $\mathbf{0 . 5 3 8}$ & $\mathbf{0 . 1 0 9}$ & $\mathbf{0 . 1 2 0}$ & 1.034 \\
MIP-1 $\alpha$ & 1.015 & 1.048 & 1.051 & 1.123 \\
TNF- $\alpha$ & 0.940 & 1.040 & 1.002 & 1.029 \\
Caspase 3 & 0.937 & 1.047 & 1.040 & 1.111 \\
\hline & & & &
\end{tabular}


Table 3.4

Normalized radiality of nodes 1-cm away from injury

\begin{tabular}{|c|c|c|c|c|}
\hline Node & $\mathbf{O} \mathbf{h}$ & $6 \mathrm{~h}$ & $24 \mathrm{~h}$ & $168 \mathrm{~h}$ \\
\hline Akt Total & 1.098 & 1.045 & 1.084 & 1.050 \\
\hline c-Jun Total & 1.072 & 1.037 & 1.058 & 1.094 \\
\hline CREB Total & 1.063 & 1.043 & 1.084 & 1.099 \\
\hline ERK1/2 Total & 1.095 & 1.056 & 1.081 & 1.017 \\
\hline JNK Total & 1.050 & 1.051 & 1.077 & 0.767 \\
\hline MEK1 Total & 0.971 & 1.055 & 1.081 & 1.090 \\
\hline p38 Total & 0.936 & 1.053 & 1.075 & 0.515 \\
\hline p53 Total & 0.950 & 1.034 & 1.079 & 0.937 \\
\hline p90RSK Total & 0.923 & 1.043 & 1.063 & 1.000 \\
\hline $\mathrm{p}-\mathrm{Akt}$ & 1.060 & 1.047 & 1.077 & 0.992 \\
\hline p-c-Jun & 1.065 & 1.046 & 1.071 & 1.037 \\
\hline p-CREB & 1.042 & 1.055 & 1.064 & 1.083 \\
\hline p-ERK1/2 & 1.068 & 1.045 & 0.948 & 1.006 \\
\hline $\mathrm{p}-\mathrm{GSK}-3 \alpha / \beta$ & 1.059 & 1.055 & 1.087 & 1.092 \\
\hline p-HSP27 & 1.078 & 1.052 & 1.077 & 1.073 \\
\hline $\mathrm{p}-\mathrm{I} \kappa \mathrm{B} \alpha$ & 1.091 & 1.052 & 1.087 & 1.066 \\
\hline p-JNK & 1.092 & 1.045 & 1.084 & 1.046 \\
\hline p-MEK1 & 1.003 & 1.042 & 0.723 & 1.057 \\
\hline p-p38 & 0.997 & 1.056 & 1.086 & 0.718 \\
\hline p-p70SK6 & 1.065 & 1.043 & 1.050 & 1.054 \\
\hline p-p90RSK & 1.085 & 1.052 & 1.087 & 1.079 \\
\hline p-STAT2 & 1.052 & 1.041 & 1.069 & 1.087 \\
\hline p-STAT3 & 1.044 & 1.045 & 0.751 & 1.079 \\
\hline IL-1 $\alpha$ & 0.992 & 0.994 & 1.054 & 0.962 \\
\hline IL-1ß & 0.735 & 0.681 & 0.890 & 0.914 \\
\hline IL-2 & 1.087 & 1.015 & 0.549 & 0.895 \\
\hline IL-6 & 0.653 & 0.102 & 0.326 & 1.014 \\
\hline MIP- $1 \alpha$ & 0.988 & 1.040 & 1.088 & 1.090 \\
\hline TNF- $\alpha$ & 0.589 & 1.028 & 1.076 & 0.988 \\
\hline Caspase 3 & 0.996 & 1.047 & 1.072 & 1.097 \\
\hline
\end{tabular}




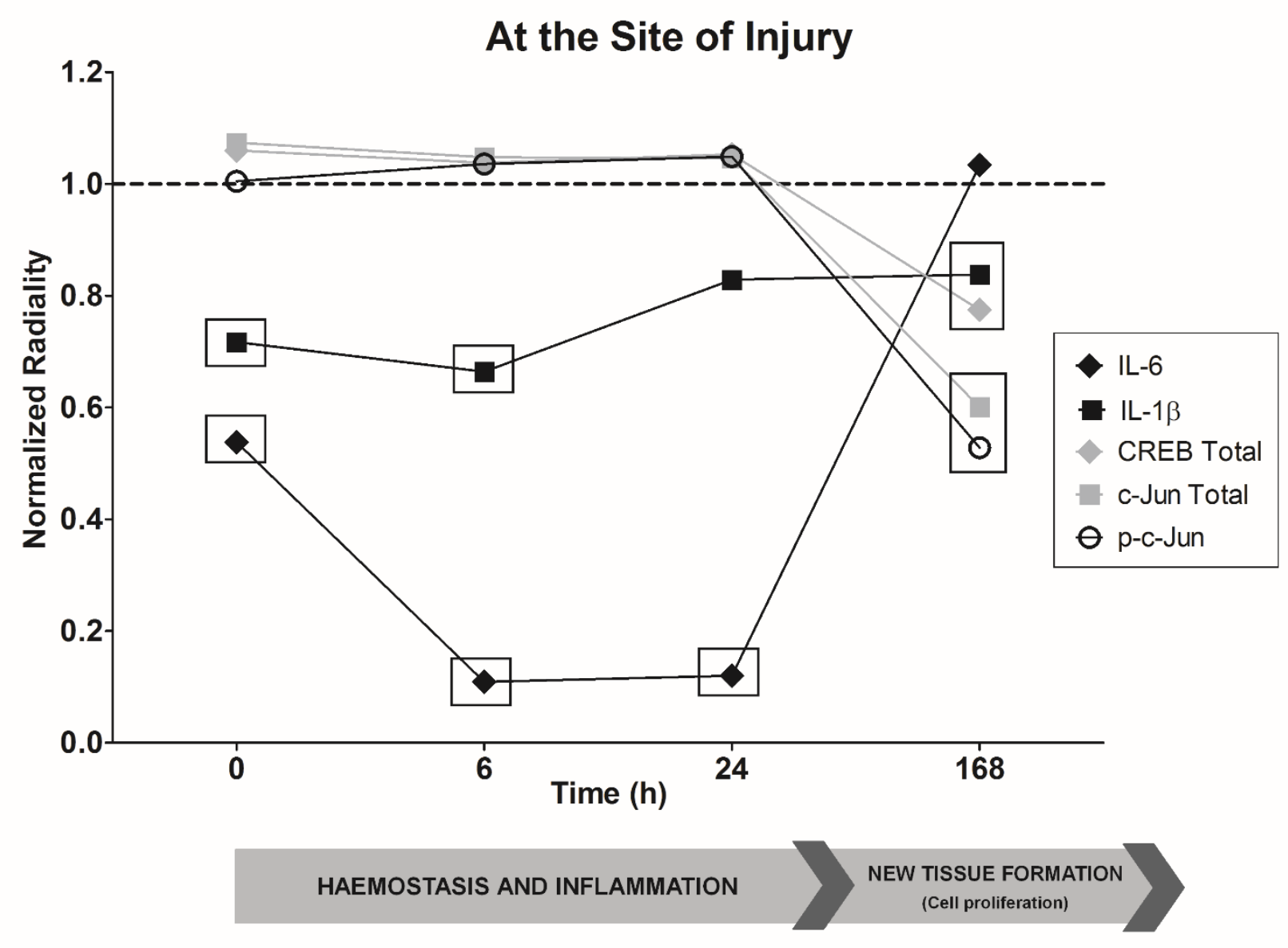

Figure 3.8. Significant radiality outcomes associated with responses at the site of fracture. Nodes that possessed significant radiality outcomes (significance threshold: average radility \pm standard deviation) were plotted over time. Five nodes (IL-6, IL-1ß, CREB, c-Jun, p-c-Jun) from networks describe responses at the site of fracture that were significant (in boxes below graph) at varying times. 


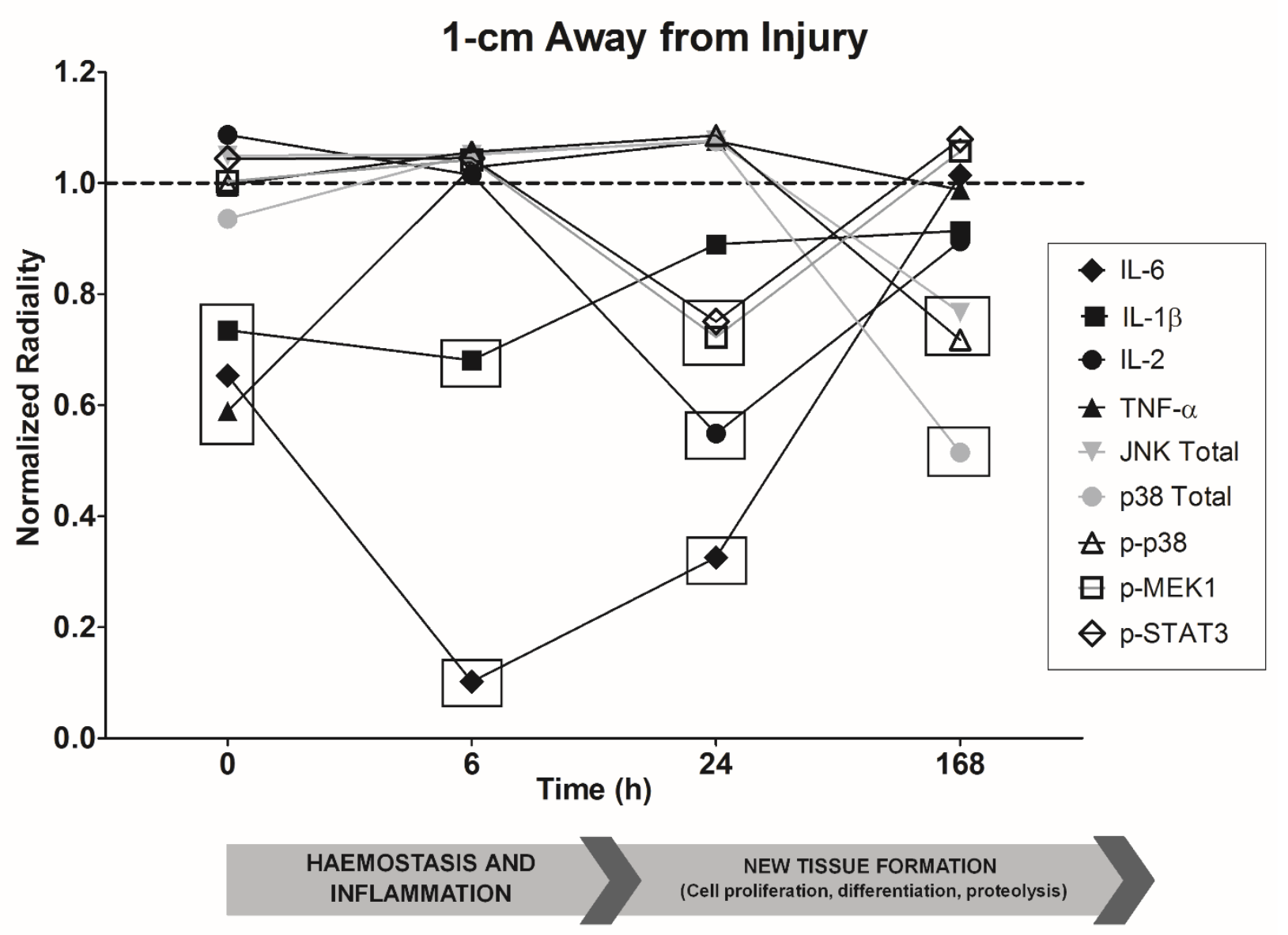

Figure 3.9. Significant radiality outcomes associated with responses 1-cm away from the site of fracture. Nodes that possessed significant radiality outcomes (significance threshold: average radility \pm standard deviation) were plotted over time. Nine nodes (IL-6, IL-1ß, IL-2, TNF- $\alpha$, JNK, p38, p-p38, p-MEK1, p-STAT3) from networks associated with injury 1-cm away from the fracture were significant (in boxes below graph) at varying times. 


\subsection{Discussion}

Biomarkers involved in wound healing processes have been examined for many types of injuries, ranging from muscle strains/contusions [16] to severe femur fractures [17]. Studies have investigated wound healing from intracellular signaling perspectives [18], spatiotemporal aspects [19], or even a genetic approach [20]. In our own previous work, we addressed the spatial and temporal distribution of cytokines, protein expression, and phosphorylation levels [5, 15], but in this study, we expanded on the research by investigating intra- and extracellular responses of an injury model with a network analysis approach in an effort to better define the significant spatiotemporal contributors of wound repair.

The cytokine, total protein, phosphorlyated protein, and Caspase-3 targets are known to be siginificant contributors to wound repair [21-27]. The responses measured in this study exhibited spatiotemporal distribution patterns that are in agreement with the current knowledge of the stages

of wound healing. Normalized concentrations of cytokines IL-1ß, IL-6, and IL-2 appeared to possess a prominent impact (significantly higher than uninjured leg) at $6 \mathrm{~h}$ post injury through 24 $\mathrm{h}$ (Figure 3.1), indicating the early repair stages of hemostasis and inflammation [28]. Additionally, most total protein (Akt, c-Jun, CREB, ERK, JNK, and p38), select phosphorylation responses (cJun, ERK1/2, MEK1, p38, p70S6K, and STAT3), and Caspase-3 activity (as shown in Figures 3.23.5) were significantly higher at the site of fracture during the later time points (24-168 h). Overall, this dataset confirms cytokines are most essential in the intermediate stages (6-24 h), while increased protein expression, phosphorylation, and caspase-3 activity are more prominent later when new tissue formation is essential. 
IPA is a valuable tool that can be used to explore signaling networks, predict which pathways and cellular processes may be involved, and visualize the connectivity within the network. Using literature references, other peripheral nodes and proteins that are likely to be involved are inserted in the network, providing researchers more potential targets to investigate. Figure 3.6 shows an IPA generated network that depicts the temporal differences $(0 \mathrm{~h}$ vs $168 \mathrm{~h})$ driven by protein, cytokine, and protease response. Figure 3.7 demonstrates the spatial difference in phosphorylation response at $6 \mathrm{~h}$, which seems to be a critical time-point, as there is a substantial change in phosphorylation signaling that occurs in tissue that suffered more damage. While IPA proved to be a useful approach to visualize the changes in response and connectivity, as well as extrapolate prospective cellular functions and mechanisms associated with the observed responses, there were a few limitations due to the nature of the experimental dataset. The complete dataset included protein expression, as well as phosphorylation of the proteins (e.g. total protein MEK1 expression and phosphorylated MEK1). To evaluate experimental responses with IPA, the data must be uploaded using identifiers supported by IPA (e.g. Genbank, UniProt/Swiss-Prot, RefSeq), meaning both protein expression and phosphorylation levels on the same protein cannot be analyzed at the same time. IPA also proposed general functions and mechanisms (e.g. cell death and survival, cellular growth and proliferation, organismal injury and abnormalities), expected for an injury model, but there were no distinct patterns that may have corresponded to specific stages of wound healing. There were also proposed networks that were not relevant to an injury model (e.g. drug metabolism), but may have shared similar molecular responses as the ones observed in this study. 
In an effort to better understand the action/reaction of the elements involved in wound healing, we applied a network analysis based on radiality, as described in the Materials and Methods section. The intent in using this analysis was not to identify direct signaling relationships, but to assess each node in comparison to all other nodes and observe how their relative responses change over time and for different zones of injury. Our results found that for most nodes normalized radiality was $\sim 1$, indicating a coordinated response by most nodes in the network. While analysis of relative responses determined that there were no significant cytokine responses at $0 \mathrm{~h}$, network analysis recognized certain cytokines to have a prominent impact in the network at the earliest time point. For instance, at time point $0 \mathrm{~h}, \mathrm{IL}-1 \beta$ and IL- 6 were significant nodes at the site of injury, and IL-1ß, IL-6, and TNF- $\alpha$ were significant nodes in tissue 1-cm away (bolded in Tables 3.3 and 3.4). However, the relative responses of these nodes were not statistically significant (different than uninjured leg) at $0 \mathrm{~h}$ (Figure 3.1), implicating cytokine activity may not be pertinent immediately following the injury and de-emphasizing their key role in the network at this time point.

While the damage was not as severe in tissue located 1-cm away from the fracture, a repair response was nonetheless exhibited. Figures 8 and 9 show the similarities and differences of networks defined by the two regions of injury, as well as a proposed progression of wound healing for these two disparate zones of injury. Time points 0-24 h post-injury capture inflammation in both regions of injury, primarily regulated by cytokines. Although markers of hemostasis were not measured in this study, it is known to be a conserved process of early wound healing, and is assumed to have occurred, considering the nature of the injury. The end of the inflammatory stage and the beginning phases of tissue formation appear to occur by $168 \mathrm{~h}$ at the site of injury, marked by the emergence of significant transcription factors (CREB, c-Jun, p-c-Jun) associated with 
wound healing, as well as the concurrent loss of cytokine significance. CREB is shown to promote satellite cell proliferation [29], while c-Jun is associated with both cell proliferation and proapoptotic roles [30]. In tissue 1-cm away, proteins associated with cell death/survival, proliferation, differentiation, and proteolysis (p-MEK1, p-STAT3, p38, p-p38, JNK) [31-35] were more relevant to the network of responses, suggesting the beginning of new tissue formation starting as early as $24 \mathrm{~h}$ and continuing through $168 \mathrm{~h}$.

Overall, centrality parameter analysis appeared to successfully identify relative responses that may hold more relevance than others, allowing us to match distinct stages of wound healing to the time points selected in this study. Many of the same conclusions, such as the importance of cytokines at early time points, were made with IPA as well. It may also be advantageous to analyze the data with both centrality parameter analysis and IPA, as there are strengths and limitations with each approach. With centrality parameter analysis, there is more control over data inclusion, but lacks the vast database integrated into IPA. In addition, the node inputs for centrality parameter analysis can be spatially disparate and thus there may, or may not, be a relationship between each pair of nodes in the network. However, in this study, we were able to treat the dataset as a single network because all of the responses were measured in the same tissues and collected from the same animals, so there is certainty that the measurements are defined by a specific time and location of injury. Nonetheless, caution must still be taken in using this approach and interpreting the results. It is also necessary to note that with many animal and time course studies, the measurements are confined to the time points selected for tissue collection and, consequently, responses that were not significant spatiotemporally or in the network analysis of this study may still be important to the injury response, but at different time points post-injury. 


\subsection{Conclusion}

In conclusion, we examined relative intra- and extracellular responses of a traumatic injury and observed certain spatiotemporal dependent patterns of cytokine, protein, phosphoprotein, and Caspase-3 activity. This injury model demonstrated phosphoprotein responses are significant immediately after the injury $(0 \mathrm{~h})$, cytokines are more critical 6-24 h, and proteins and Caspase-3 become more relevant torwards the later times of wound healing. Network centrality parameter analysis identified significant outomes that were not apparent with relative response data, and allowed us to discern the different stages of wound healing that occurred at certain time points and regions of injury. Severely damaged tissue showed molecular indications of inflammation, and new tissue formation by $168 \mathrm{~h}$ post-injury, while muscle tissue further from the site of injury (that experienced less damage) may have progressed to new tissue formation as early as $24 \mathrm{~h}$ and proceeded to other processes, such as cell differentiation and proteolysis, by $168 \mathrm{~h}$. This study demonstrated great potential in using a network centrality parameter analysis approach to investigate relative cellular responses in different stages of wound healing, and to monitor changes under varying spatial and temporal conditions, thereby allowing for the identification of new targets timed for specific injury patterns.

\subsection{Acknowledgements}

This research was conducted with support from a grant awarded by The Brodie Discovery and Innovation Fund. 


\subsection{References}

[1] Behm B, Babilas P, Landthaler M, Schreml S. Cytokines, chemokines and growth factors in wound healing. J Eur Acad Dermatol Venereol. 2012;26(7):812-20.

[2] Aronson D, Wojtaszewski JF, Thorell A, Nygren J, Zangen D, Richter EA, et al. Extracellularregulated protein kinase cascades are activated in response to injury in human skeletal muscle. Am J Physiol. 1998;275(2 Pt 1):C555-61.

[3] Velnar T, Bailey T, Smrkolj V. The wound healing process: an overview of the cellular and molecular mechanisms. J Int Med Res. 2009;37(5):1528-42.

[4] Stroncek JD, Reichert WM. Overview of Wound Healing in Different Tissue Types. In: W.M. Reichert, editor. Indwelling Neural Implants: Strategies for Contending with the In Vivo Environment. Boca Raton (FL): CRC Press/Taylor \& Francis; 2008. Chapter 1.

[5] Currie HN, Loos MS, Vrana JA, Dragan K, Boyd JW. Spatial cytokine distribution following traumatic injury. Cytokine. 2014;66(2):112-8.

[6] Shi Y, Shu B, Yang R, Xu Y, Xing B, Liu J, et al. Wnt and Notch signaling pathway involved in wound healing by targeting c-Myc and Hes1 separately. Stem Cell Res Ther. 2015;6:120.

[7] Bielefeld KA, Amini-Nik S, Alman BA. Cutaneous wound healing: recruiting developmental pathways for regeneration. Cell Mol Life Sci. 2013;70(12):2059-81.

[8] Arodz T, Bonchev D, Diegelmann RF. A Network Approach to Wound Healing. Adv Wound Care. 2013;2(9):499-509.

[9] Morel PA, Lee RE, Faeder JR. Demystifying the cytokine network: Mathematical models point the way. Cytokine. 2016. 
[10] Thomas S, Bonchev D. A survey of current software for network analysis in molecular biology. Hum Genomics. 2010;4(5):353-360.

[11] van Riel NA. Dynamic modelling and analysis of biochemical networks: mechanism-based models and model-based experiments. Brief Bioinform. 2006;7(4):364-74.

[12] Abedi M, Gheisari Y. Nodes with high centrality in protein interaction networks are responsible for driving signaling pathways in diabetic nephropathy. PeerJ. 2015.

[13] Currie HN, Vrana JA, Han AA, Scardoni G, Boggs N, Boyd JW. An Approach to Investigate Intracellular Protein Network Responses. Chem Res Toxicol. 2014;27(1):17-26.

[14] Scardoni G, Laudanna C. Centralities Based Analysis of Complex Networks. In: Zhang DY (Ed.). New Frontiers in Graph Theory 2012.

[15] Han AA, Currie HN, Loos MS, Vrana JA, Fabyanic EB, Prediger MS, et al. Spatiotemporal phosphoprotein distribution and associated cytokine response of a traumatic injury. Cytokine. 2016;79:12-22.

[16] Jarvinen TA, Jarvinen TL, Kaariainen M, Kalimo H, Jarvinen M. Muscle injuries: biology and treatment. Am J Sports Med. 2005;33(5):745-64.

[17] Schindeler A, McDonald MM, Bokko P, Little DG. Bone remodeling during fracture repair: The cellular picture. Semin Cell Dev Biol. 2008;19(5):459-66.

[18] Guan CX, Cui YR, Zhang M, Bai HB, Khunkhun R, Fang X. Intracellular signaling molecules involved in vasoactive intestinal peptide-mediated wound healing in human bronchial epithelial cells. Peptides. 2007;28(9):1667-73. 
[19] Chamberlain CS, Crowley E, Vanderby R. The spatio-temporal dynamics of ligament healing. Wound Repair Regen. 2009;17(2):206-15.

[20] Li X, Gu W, Masinde G, Hamilton-Ulland M, Xu S, Mohan S, et al. Genetic control of the rate of wound healing in mice. Heredity (Edinb). 2001;86(Pt 6):668-74.

[21] L. Holmdahl L, Ivarsson ML. The role of cytokines, coagulation, and fibrinolysis in peritoneal tissue repair. Eur J Surg. 1999;165(11):1012-9.

[22] Song G, Ouyang G, Bao S. The activation of Akt/PKB signaling pathway and cell survival. J Cell Mol Med. 2005;9(1):59-71.

[23] Mebratu Y, Tesfaigzi Y. How ERK1/2 activation controls cell proliferation and cell death: Is subcellular localization the answer? Cell Cycle. 2009;8(8):1168-75.

[24] Cross TG, Scheel-Toellner D, Henriquez NV, Deacon E, Salmon M, Lord JM. Serine/threonine protein kinases and apoptosis. Exp Cell Res. 2000;256(1):34-41.

[25] Basson MA. Signaling in cell differentiation and morphogenesis. Cold Spring Harb Perspect Biol. 2012;4(6).

[26] Arya AK, Tripathi R, Kumar S, Tripathi K. Recent advances on the association of apoptosis in chronic non healing diabetic wound. World J Diabetes. 2014;5(6):756-62.

[27] Fernando P, Kelly JF, Balazsi K, Slack RS, Megeney LA. Caspase 3 activity is required for skeletal muscle differentiation. Proc Natl Acad Sci U S A. 2002;99(17):11025-30.

[28] Margetic S. Inflammation and haemostasis. Biochem Med (Zagreb). 2012;22(1):49-62.

[29] Stewart R, Flechner L, Montminy M, Berdeaux R. CREB is activated by muscle injury and promotes muscle regeneration. PLoS One. 2011;6(9):e24714. 
[30] Shaulian E, Karin M. AP-1 in cell proliferation and survival. Oncogene. 2001;20(19):2390400.

[31] Thomas S, Bonchev D. A survey of current software for network analysis in molecular biology. Hum Genomics. 2010;4(5):353-60.

[32] Zhang W, Liu HT. MAPK signal pathways in the regulation of cell proliferation in mammalian cells. Cell Res. 2002;12(1):9-18.

[33] Zhu H, Xiao F, Wang G, Wei X, Jiang L, Chen Y, et al. STAT3 Regulates Self-Renewal of Adult Muscle Satellite Cells during Injury-Induced Muscle Regeneration. Cell Rep. 2016;16(8):2102-15.

[34] Hoene M, Runge H, Haring HU, Schleicher ED, Weigert C. Interleukin-6 promotes myogenic differentiation of mouse skeletal muscle cells: role of the STAT3 pathway. Am J Physiol Cell Physiol. 2013;304(2):C128-36.

[35] Silva KA, Dong J, Dong Y, Schor N, Tweardy DJ, Zhang L, et al. Inhibition of Stat3 activation suppresses caspase-3 and the ubiquitin-proteasome system, leading to preservation of muscle mass in cancer cachexia. J Biol Chem. 2015;290(17):11177-87. 


\section{Chapter 4}

The investigation of salivary cytokines of individual and shared success and failure experiences 


\section{The investigation of salivary cytokine response to individual and shared success and failure experiences}

Saliva is viewed as a convenient diagnostic medium for its advantages in ease of collection and handling, low risk and inconvenience, and as a minimally-invasive approach. Regulated by the autonomic system, salivary glands can secrete an assortment of proteins, hormones, and immunoglobulins that can potentially serve as diagnostic tool for a variety of diseases, disorders, or exposures. In particular, salivary cytokines have been investigated to understand alterations involving the immune and inflammatory response. This study examined the production of 9 cytokines (IL-2, IFN- $\gamma$, IL-10, IL-5, IL-6, TNF- $\alpha$, IL-1 $\beta$, IL-4, GM-CSF) as subjects experienced a variety of stressors individually and as a group. By monitoring salivary cytokine production immediately following exposure to stressors, certain trends that reflect adaptations in the stress response have been identified. Notably, overall cytokine response increased when subjects experienced negative stress (failure), compared to positive stress (success). However, cytokine levels were not significantly altered $(p>0.05)$ when the subjects encountered either positive or negative stress as a team (i.e. responses were not different than the baseline). These results probe the idea that stress can be distributed among multiple individuals. Additionally, even with the potential challenges of subject population variation, principal component analysis and hierarchal clustering verified the capacity of employing cytokine measurements to characterize the stress response. 


\subsection{Introduction}

Salivary biomarkers are becoming an increasingly investigated measurement in diagnosing, screening, or monitoring the effects of adverse events [1]. Analyses of saliva samples have demonstrated the capacity to evaluate a range of conditions, such as oral cancer [2], radiation exposure [3], acute stress [4], and various systemic diseases [5]. The advantages of using saliva over other biological samples are apparent in its minimally-invasive approach, ease of collection and handling, and low risk and inconvenience to the subject and investigator. Regulated by the autonomic nervous system, stimulated saliva can be influenced by chewing, pain, medication, systemic diseases [6], and even stress [7]. The composition of whole saliva (stimulated and unstimulated saliva) is primarily water (99\%), followed by an assortment of proteins, cystatins, mucins, histatins, amylases, statherin [8], electrolytes, immunoglobulins, enzymes, and nitrogenous species [6]. As salivary glands are mediated by parasympathetic and sympathetic nerves, the concentrations of these components found in saliva (i.e. protein secretion) may be attributed to signaling mechanisms controlled by the autonomic nervous system [9].

As central intercellular regulators of the immune and inflammatory response, cytokine biomarkers possess the potential to serve as diagnostic tools for a wide range of diseases and disorders [10]. In particular, the use of salivary cytokines to diagnosis oral health, including squamous cell carcinoma [11] and periodontitis [12], have been investigated. While salivary cytokine biomarkers are generally explored to evaluate oral conditions and diseases, they have also been shown to be associated with conditions not localized in the mouth, such as chronic kidney disease [13] and diabetes [14]. There is additionally a growing interest in using salivary cytokines to monitor inflammatory immune responses [15]. For instance, Sjögren's Syndrome is a systemic 
chronic inflammatory autoimmune disorder that has been characterized by an abnormal profile of pro- and anti-inflammatory salivary cytokines [16].

While the literature is limited at this time, recent studies have observed alterations in salivary cytokine concentrations that may even be linked to acute social-cognitive and physical stressors [17], and further described these responses to potentially impact overall health [18]: extreme changes in inflammatory cytokine levels have been associated with ailments, such as depression [19]. Examining the cytokine response resulting from both positive and negative stress may offer insight into the future implications of different types of stress, along with the factors that influence the overall response. In particular, this study examined if salivary cytokine responses reflect how individuals in stressful environments perceive success and failure, and how their responses are affected when success and failure are shared among other individuals. Research on general stress biomarkers [20-23], and how team dynamics are influenced by stress is extensive [24-26], but using salivary biomarkers to quantitatively assess differences influenced by social constructs, which may contribute to or alleviate stress, is still a developing approach. Furthermore, exploring salivary cytokines may enhance our understanding of the involvement of the immune and inflammatory system in the stress response and the distribution of stress. 


\subsection{Materials and methods}

\subsubsection{Materials}

Salimetrics Collection Aid® (Salimetrics LLC, State College, PA) were purchased to assist with saliva collection via passive drooling. Cytokines were measured with the Human Ultrasensitive Cytokine Magnetic 10-Plex Panel kit (Invitrogen, Carlsbad, CA; Catalog \# LHC6004M).

\subsubsection{Subjects}

Participants were recruited from the Air Force Reserve Officers' Training Corps at West Virginia University. The subjects were between the ages of 18-22, consisting of 16 males and 1 female. One male subject was removed from the dataset due to incompletion of the study and data for 2 male subjects were excluded due to insufficient saliva sample delivery. The female subject was not omitted from the cohort, as inclusion of the subject's data did not significantly alter the results. All protocols for this study were reviewed and approved by the Institutional Review Board at West Virginia University and USAMRMC Office of Research Protections. Prior to proceeding with the study, the principal investigator informed the subjects of the experimental design, potential risks associated with the study, and assured confidentiality of any identifiers. Subjects then provided written and informed consent to be willing test subjects, with the option to withdraw consent to participate at any time. Profiles of each subject (Sex, age, race, weight, height) are presented in Table 4.1. 
Table 4.1. Subject Information

\begin{tabular}{ccccccc}
\hline Team & Subject & Sex & Age & Race & $\begin{array}{c}\text { Weight } \\
\text { (lb.) }\end{array}$ & $\begin{array}{c}\text { Height } \\
\text { (in.) }\end{array}$ \\
\hline \multirow{3}{*}{ Team 1 } & 1 & M & 22 & White & 139 & 64 \\
& 2 & M & 19 & White & 212.2 & 72.5 \\
& 3 & M & 18 & White & 151.6 & 69 \\
Team 2 & 4 & M & 19 & White & 119.2 & 67.5 \\
& 5 & M & 20 & White & 199.2 & 76 \\
& 6 & M & 18 & White & 205 & 75 \\
Team 3 & 7 & M & 19 & White & 156.4 & 70 \\
& 9 & M & 20 & White & 223.6 & 76.5 \\
& 10 & M & 19 & White & 188.6 & 74.5 \\
& 11 & M & 19 & White & 157.2 & 68 \\
Team 4 & 12 & M & 19 & White & 151.6 & 70 \\
& 13 & F & 21 & White & 163.8 & 72.5 \\
& 14 & M & 21 & White & 156.2 & 66.5 \\
& & & & & & 73 \\
\hline
\end{tabular}

\subsubsection{Experimental design: mock hostage rescue}

Each subject was tasked to perform a mock hostage rescue mission, simulating features of physical, emotional, cognitive, and even social stressors experienced by U.S. Military Special Operations Forces and Hostage Rescue Teams of the Federal Bureau of Investigation. The mock rescue was arranged inside the Crime Scene Training Complex (CSTC), provided by the Department of Forensic and Investigative Science at West Virginia University. First, the subjects were given one minute to read a written brief of the mission immediately before entering the complex. Subjects were informed that the CSTC is a known terrorist stronghold and possible bomb-making facility. After monitoring the facility for some time, the structure was assumed to be vacant. It was, however, believed that a hostage, who may know valuable information, was 
imprisoned inside. The objective of the mission was to carefully canvass the building and rescue the "hostage" (70 pound punching bag). Subjects were told that the hostage may be injured and possibly unconscious, requiring the search and utilization of a backboard, hand cart, and four bungee cords to safely transport the hostage to the rendezvous point, outside of the complex. All four items (hostage, backboard, hand cart, and bungee cords) were spread out throughout the building. In addition to completing the rescue, the subjects were directed to leave no evidence of their presence (i.e. lights off, close all doors).

The subjects performed the mock rescue multiple times. For one attempt, they were instructed to complete the mission as fast as they could. For the subsequent attempt, subjects were again instructed to execute the mission as fast as they could, but 1 minute after entering the premises, they were interrupted with an urgent message that armed insurgents were returning to the complex in 1 minute. If the subjects could not rescue the hostage and exit the building before the insurgents returned, they would be captured and fail the mission. These two situations were designed to ensure that the subjects would experience both success and planned failure in attempting the rescue. Subjects were instructed to continue with the completion of the mission after being told they failed the rescue. The study additionally required the subjects to be present at the CSTC for two separate occasions: The subjects first participated in the experiments individually and then returned on a later date to perform the tasks in teams. The teams were randomly generated, but adjusted for any scheduling and availability conflicts. Individual performance did not influence how the teams were formed. All teams were made up of 4 subjects, but one subject from Teams 1 and 2 had to be removed from the dataset due to insufficient saliva 
sample delivery. Team 1 consisted of Subjects 1-3; Team 2 consisted of Subjects 4-6; Team 3 consisted of Subjects 7-10; and Team 4 consisted of Subjects 11-14.

\subsubsection{Saliva sample collection and analysis}

Saliva samples were collected prior to beginning the experiment (before subjects were informed of the mission), and immediately after performing the successful and failed rescues. Approximately $1 \mathrm{~mL}$ of saliva was dispensed into a $1.5 \mathrm{~mL}$ cryogenic tube, using the passive drooling method (Salimetrics, 2011). All samples were placed in ice for 3-4 hours at the CSTC and transferred to $-80^{\circ} \mathrm{C}$ storage until the time of analysis. Saliva samples were analyzed for 10 cytokines (IL-2, IFN- $\gamma$, IL-10, IL-5, IL-6, TNF- $\alpha$, IL-1 $\beta$, IL-4, GM-CSF and IL-8), using the Human Ultrasensitive Cytokine Magnetic 10-Plex Panel kit. IL-8 data were omitted, as nearly 90\% of the measurements were above the upper limit of quantitation and limited saliva sample volume prevented further analysis with diluted samples. Fluorescence intensities were taken with the BioPlex 200 Luminex system and Pro II Wash Station (Bio-Rad, Hercules, CA) according to the manufacturer's instructions. Salivary cortisol levels were also measured with the Cortisol EIA kit (Cayman Chemical, Catalog No. 582121). Absorbance readings were taken with the Infinite M1000 microplate reader (Tecan US, Raleigh NC, USA). All measurements were taken in triplicate and cytokine or cortisol concentrations (pg/mL) were determined according to 5parameter logistic regression curves constructed from standards in the kit. Grubb's test was employed to identify and remove any outliers. 


\subsubsection{Statistical analysis}

Data were analyzed using Prism 5 (GraphPad, La Jolla, CA). Baseline, post-success and post-fail concentrations $(\mathrm{pg} / \mathrm{mL})$ for each cytokine or cortisol were measured after individual and team performances. All cytokine concentrations were normalized to the baseline (cytokine level in saliva collected prior to mission briefing) and averaged for all 14 subjects. Normalized cytokine responses were additionally analyzed for each individual subject. Significant differences among any response resulting from individual versus team performance and baseline versus success versus failure responses were determined with 2-way ANOVA with Bonferroni post-hoc test, where $\mathrm{p}<0.05$ was considered significant.

Principal component analysis (PCA) and unsupervised 2-way hierarchical cluster analysis with Ward's minimum variance were performed using SAS JMP Pro 12.0.1 (Carey, NC). PCA and cluster analyses were performed on the nine variables (normalized cytokine levels) expressed by all 14 subjects, following successful and failed performances, experienced as individuals and teams. PCA reorganizes and simplifies complex datasets containing a large number factors (e.g. cytokine responses) by generating new variables (principal components) that are computed based on the variability within the dataset. The principal components were created using the sum of squares and cross products matrix for the unscaled and uncentered data. Score and loading plots of the analyses, along with the percentages of the variation covered by each principal component, are reported. Hierarchical cluster analysis groups responses (e.g. cytokine levels) that are similar, based on the distances between pairs of clusters. The distance between two clusters is defined by the ANOVA sum of squares and with every round of clustering, two groups that have the smallest 
distance in between them are combined together to form one cluster. This operation continues until all data points have been placed into a single cluster.

\subsection{Results}

\subsubsection{Normalized salivary cytokine responses of individual and team performances}

Normalized concentrations of 9 cytokines (IL-2, IFN- $\gamma$, IL-10, IL-5, IL-6, TNF- $\alpha$, IL-1 $\beta$, IL-4, and GM-CSF) were measured in saliva collected from 14 subjects and averaged, as they performed the tasks individually and in teams (Figure 4.1). When the subjects completed the missions individually, there was a general increase (normalized value $>1$ ) in cytokine response after experiencing both success and failure, indicating an increase in cytokine response from baseline levels. Normalized values ranged from $1.21 \pm 0.17$ to $2.24 \pm 0.52$ after completing successful tasks and $1.90 \pm .36$ to $4.76 \pm 1.08$ for cytokines measured after failed tasks. Additionally, post-fail levels were overall further elevated, compared to post-success responses: statistically significant increases were observed for IL-2, GM-CSF, IL-4, and IL-10 measurements.

Post-success cytokine levels following team performance were also increased, ranging from $1.04 \pm 0.10$ to $1.53 \pm 0.34$, while post-fail responses were comparable to baseline levels, ranging from $0.97 \pm 0.07$ to $1.12 \pm 0.16$. There were no significant differences between success and failure associated cytokine responses when the subjects worked in teams. Evaluation of individual versus team responses found no significant differences in cytokine levels resulting from successful tasks. There was, however, a clear significant decrease in post-fail levels for all cytokines (except IL-1 $\beta$ ) during team performance, compared to individual cytokine response. 
Normalized cytokine levels for each individual subject are provided in Appendix B. Varied responses were observed among the subjects.

\section{Average Normalized Cytokine Response}
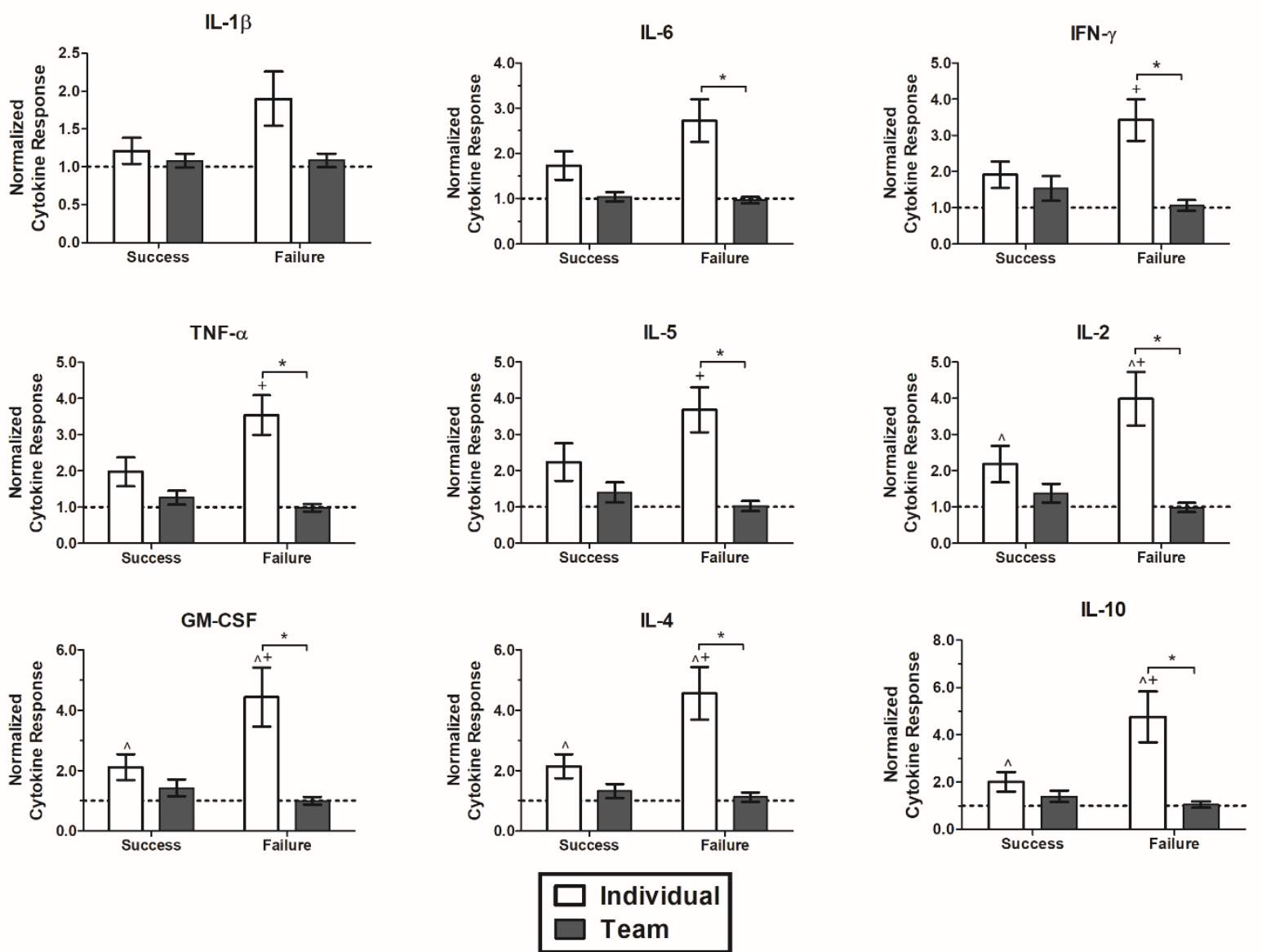

Figure 4.1. Normalized cytokine response following team and individual performance. Salivary cytokine (IL-1 $\beta$, IL-6, IFN- $\gamma$, TNF- $\alpha$, IL-5, IL-2, GM-CSF, IL-4, and IL-10) levels were determined for each subject after experiencing success and failure as an individual and as a team. All responses were normalized to baseline concentrations measured prior to beginning the sessions. The displayed cytokine responses are averages of the normalized levels for all subjects $(\mathrm{N}=14)$. Error bars reflect \pm standard error of the mean due to the variation in response among individuals. Statistically significant differences between success and failure associated levels are marked with a matching symbol $\left({ }^{\wedge}\right)$ and significant differences between individual and team performance are notated with the symbol *. + indicates a significant change, compared to the baseline (dashed line indicates normalized response $=1)$. Statistically significant $(\mathrm{p}<0.05)$ differences were determined by 2-way ANOVA with a Bonferroni post-test. 


\subsubsection{Task completion times}

The time taken for each subject to complete the tasks as an individual and as a team were recorded (Table 4.2). Completion of individual sessions for successful performances ranged from 4 minutes and 45 seconds - 9 minutes and 3 seconds, with an average time of 6 minutes and 22 seconds. Individual failure times were similar, with a range of 4 minutes and 44 seconds - 10 minutes and 41 seconds and an average of 7 minutes and 1 second. When the subjects performed as teams, the times were overall increased. Team success times ranged from 6 minutes and 15 seconds - 18 minutes and 49 seconds, with an average of 12 minutes and 35 seconds, while team failure times ranged from 11 minutes and 48 seconds - 27 minutes and 26 seconds, with an average of 17 minutes and 8 seconds. While all subjects underwent planned failure in the same time span ( 2 minutes after starting the mission), the recorded times illustrate how some subjects took more time to complete the tasks after being told they had failed.

Table 4.2. Task completion times (hh:mm:ss)

\begin{tabular}{|c|c|c|c|c|c|}
\hline & & \multicolumn{2}{|c|}{ INDIVIDUAL } & \multicolumn{2}{|c|}{ TEAM } \\
\hline & & Success & Fail & Success & Fail \\
\hline \multirow{3}{*}{ Team 1} & Subject 1 & $0: 05: 13$ & 0:08:18 & $0: 16: 15$ & $0: 11: 48$ \\
\hline & Subject 2 & $0: 04: 45$ & 0:06:58 & & \\
\hline & Subject 3 & 0:05:08 & 0:05:48 & & \\
\hline \multirow[t]{3}{*}{ Team 2} & Subject 4 & $0: 08: 12$ & $0: 10: 41$ & $0: 08: 49$ & $0: 18: 22$ \\
\hline & Subject 5 & 0:06:07 & 0:05:29 & & \\
\hline & Subject 6 & 0:06:14 & 0:08:57 & & \\
\hline \multirow[t]{4}{*}{ Team 3} & Subject 7 & 0:06:54 & 0:04:44 & 0:06:55 & 0:10:57 \\
\hline & Subject 8 & 0:06:39 & 0:05:41 & & \\
\hline & Subject 9 & 0:06:37 & 0:05:04 & & \\
\hline & Subject 10 & 0:04:51 & 0:06:46 & & \\
\hline \multirow{5}{*}{ Team 4} & Subject 11 & 0:09:03 & 0:09:39 & $0: 18: 21$ & $0: 27: 26$ \\
\hline & Subject 12 & $0: 08: 16$ & 0:09:46 & & \\
\hline & Subject 13 & 0:05:51 & 0:05:11 & & \\
\hline & Subject 14 & 0:05:24 & 0:05:07 & & \\
\hline & Average & 0:06:22 & 0:07:01 & 0:12:35 & 0:17:08 \\
\hline
\end{tabular}




\subsubsection{Principal component analysis score and loading plots}

PCA was performed to describe the variance among normalized cytokine responses resulting from experiencing success and failure, as an individual or within a team. Score and loading plots generated from individual and team based datasets are shown in Figures 4.2 and 4.3, respectively. Blue color-coded variables represent responses associated with success, while the red color-coded variables signify responses resulting from failed performances. Principal components (PC) 1 and 2 from the analysis of individual data covered 96.46\% of the variance among the cytokine responses. The score plot exhibited a clear distinction between cytokine responses resulting from success and failure, with a tighter grouping for success responses. The loading plot revealed that the contribution of the different subjects to the variance of PC 1 was similar (values ranged from 0.869 to 0.988 ) and significant (the closer the value is to 1 the greater the effect of the component on the variable). For PC 2, certain subjects contributed more to the variance, particularly subjects 7 and 14 (value $>0.3$ ).

PC 1 and 2 from the analysis of team data described $98.77 \%$ of the variance among the cytokine responses. In contrast to the PCA results of the individual responses, the score plot showed a tighter grouping for failure associated cytokine response, while success associated responses were more varied. In fact, the IL-1 $\beta$ and IL-6 levels measured after successful performances appeared to fit with the cytokine profile related to failure. The loading plot revealed that all subjects contributed to the variance of PC 1 similarly (values ranged from 0.826 to 0.992 ) and significantly (values are close to 1). For PC 2, certain subjects contributed more to the variance, particularly subjects $7,5,6$, and 3 (value $>0.3$ ). 

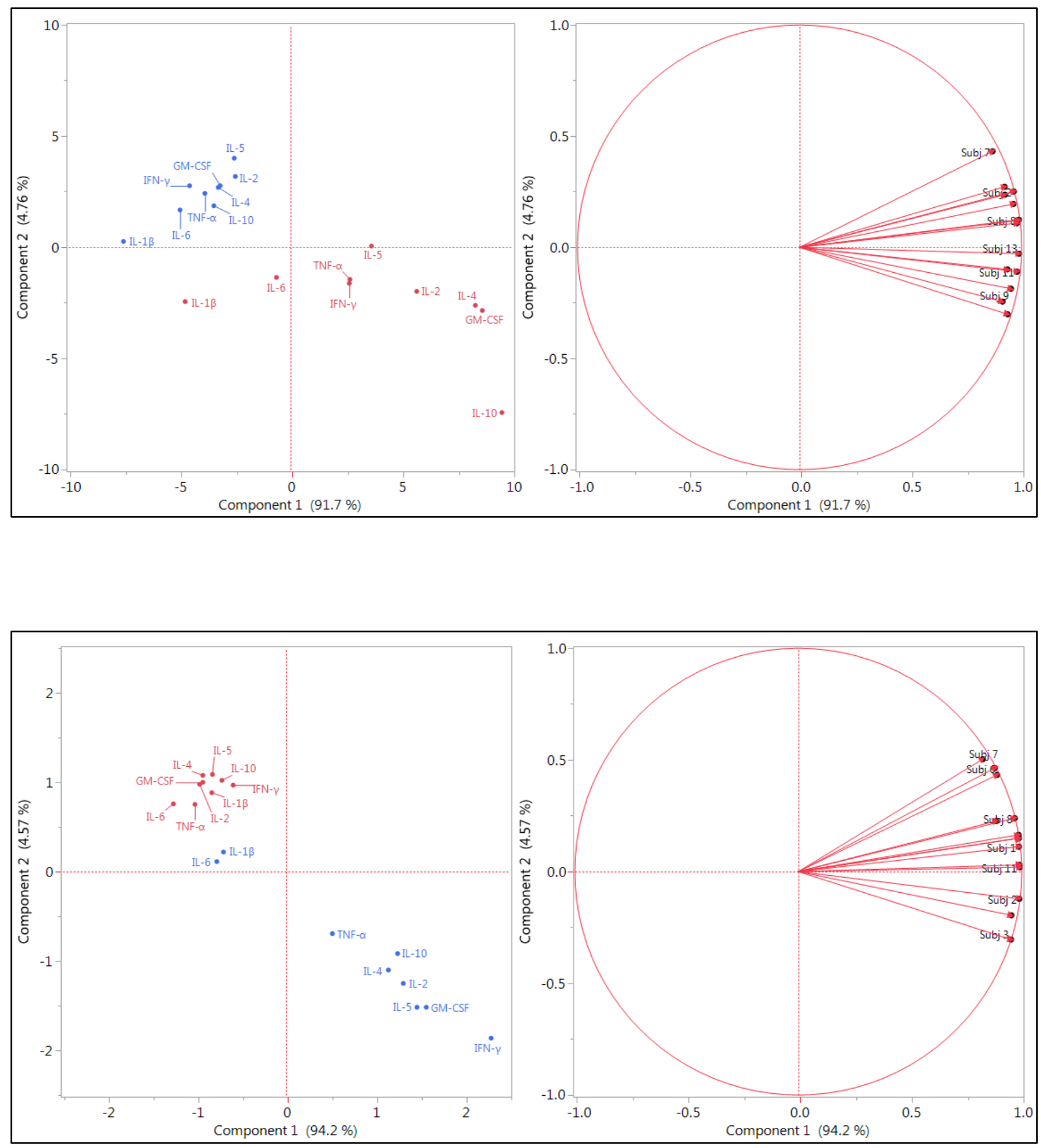

Figures 4.2. and 4.3. PCA: score and loading plots of cytokine response. PCA generated score and loading plots using individual (top) and team (bottom) datasets are shown. Blue color-coded variables represent responses associated with success, while the red color-coded variables signify responses resulting from failed performances. 


\subsubsection{Hierarchical cluster analysis with Ward's minimum variance}

2-way hierarchical clustering of the 9 cytokine responses associated with individual and team efforts are shown in Figures 4.4 and 5.5, respectively. The red-labeled cytokines represent the responses resulting from failure, while the blue-labeled cytokines indicate responses due to successful experiences. During individual performances, there was a distinct clustering of all success associated cytokines. Cytokine responses due to failure also clustered well, except for IL-6, IL-1 $\beta$, and TNF- $\alpha$, which appeared to be on the border between joining the success or failure clusters.
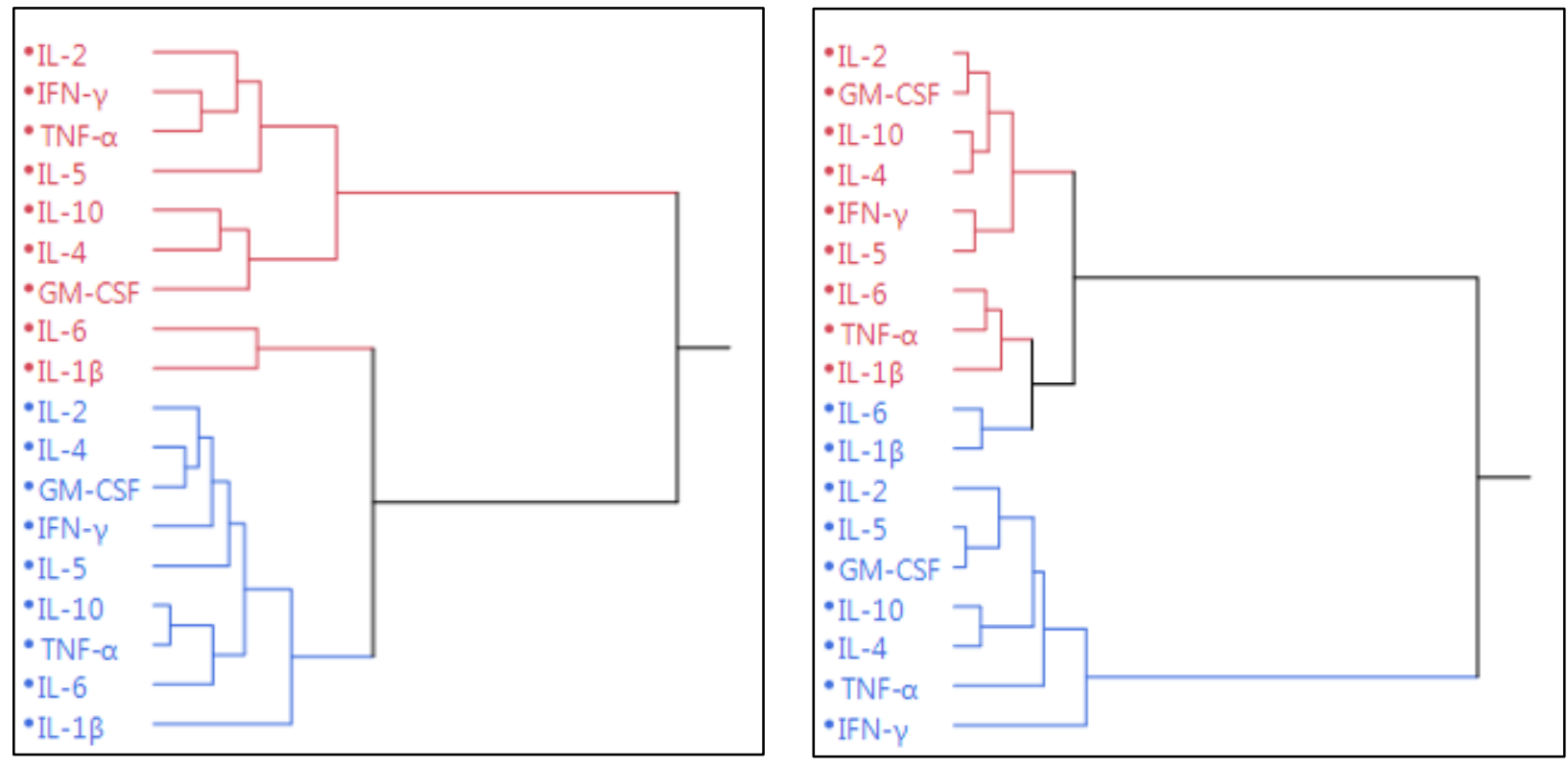

Figures 4.4. and 4.5. 2-way hierarchical cluster analysis with Ward's minimum variance. Clustering analysis performed with individual (left) and team (right) responses are shown. Blue color-coded variables represent responses associated with success, while the red color-coded variables signify responses resulting from failed performances. 


\subsubsection{Baseline cytokine concentrations}

Baseline concentrations $(\mathrm{pg} / \mathrm{mL})$ for all cytokines, averaged for all subjects are shown in Table 4.3. The baseline samples were collected at the beginning the individual and team experiments (prior to the mission briefing). Overall, all baseline cytokine levels from team performances were higher than baseline levels from individual performances. Only IL-1 $\beta$, IL-6, TNF- $\alpha$, and GM-CSF concentrations, however, were statistically significantly elevated $(p<0.05)$.

Table 4.3. Averaged baseline cytokine concentrations

\begin{tabular}{lccccc}
\hline CYTOKINE & \multicolumn{2}{c}{ INDIVIDUAL } & \multicolumn{2}{c}{ TEAM } & \\
\hline & $\begin{array}{c}\text { Concentration } \\
(\mathrm{pg} / \mathrm{mL})\end{array}$ & S.E.M. & $\begin{array}{c}\text { Concentration } \\
(\mathrm{pg} / \mathrm{mL})\end{array}$ & S.E.M. & $\mathrm{p}$ value* \\
IL-1b & 171.1 & 45.8 & 309.3 & 44.0 & $\mathbf{p}<\mathbf{0 . 0 5}$ \\
IL10 & 3.1 & 1.1 & 150.4 & 92.9 & $\mathrm{p}>0.05$ \\
IL6 & 54.1 & 26.2 & 215.1 & 24.8 & $\mathbf{p}<\mathbf{0 . 0 5}$ \\
GM-CSF & 75.4 & 54.6 & 389.8 & 89.3 & $\mathbf{p}<\mathbf{0 . 0 5}$ \\
IL5 & 4.2 & 1.8 & 177.8 & 88.1 & $\mathrm{p}>0.05$ \\
IFN-g & 24.4 & 20.5 & 104.3 & 33.6 & $\mathrm{p}>0.05$ \\
TNF-a & 17.9 & 11.2 & 146.1 & 45.1 & $\mathbf{p}<\mathbf{0 . 0 5}$ \\
IL2 & 3.0 & 1.2 & 108.4 & 52.1 & $\mathrm{p}>0.05$ \\
IL4 & 73.0 & 58.7 & 291.2 & 88.6 & $\mathrm{p}>0.05$ \\
\hline
\end{tabular}

*p value signifies statistical significance between individual and team cytokine concentration. S.E.M. $=$ Standard error of the mean. $\mathrm{N}=14$.

\subsubsection{Success and failure associated salivary cortisol concentrations}

Averaged salivary cortisol concentrations are shown in Table 4.4. There were no differences in cortisol concentration resulting from success or failure whether the subjects worked 
as an individual or as a team. There appeared to be an overall decrease in cortisol levels when the subjects were placed in teams. However, these differences were not statistically significant.

Table 4.4. Averaged salivary cortisol concentrations

\begin{tabular}{lccccc}
\hline & $\begin{array}{c}\text { Concentration } \\
(\mathrm{pg} / \mathrm{mL})\end{array}$ & S.E.M. & & $\begin{array}{c}\text { Concentration } \\
(\mathrm{pg} / \mathrm{mL})\end{array}$ & S.E.M. \\
\hline & & & & & \\
Individual Success & 595.8 & 282.9 & Team Success & 222.5 & 43.8 \\
Individual Failure & 568.4 & 270.1 & Team Failure & 188.1 & 29.5 \\
\hline
\end{tabular}

S.E.M. $=$ Standard error of the mean. $\mathrm{N}=14$.

\subsection{Discussion}

The understanding of the salivary cytokine response resulting from stress is advancing, offering a potential minimally-invasive approach to analytically monitor and evaluate the stress response $[27,28]$. As the effects of high intensity and chronic stress can be deleterious to one's health, observing the changes in cytokine levels can lend insight into the relationship between the inflammatory and immune response. Certain cytokines, such as IL-6 and IL-10, appear to exhibit a generally consistent pattern of increased concentration as a result of stress exposure [29, 30], but these patterns can be biased by the many discrepancies in the types and degrees of stressors, time points at which the cytokines are measured, or even the number of studies investigating specific cytokines. Therefore, a current challenge is to distinguish which factors actually contribute to the observed cytokine response. In addition, cytokines appear to be ubiquitous biomarkers for many different diseases [31-33] or exposures [34], further increasing the difficulty in discerning which cytokine responses are relevant to the focus of investigation. Then the overarching goal of this 
research, and for all future related research, is to offer insight into the complex cytokine response system and to distinguish cytokine trends associated with various stress conditions.

The central objective of this study was to assess measurable biochemical responses to stress, influenced by social factors. In particular, the premise of individuals experiencing and reacting to stressors together as a group and how the outcome differs from individuals facing the same stressors alone was investigated. Normalized cytokine responses associated with either positive stress (success) or negative stress (failure) were not significantly influenced ( $p>0.05)$ when the subjects encountered the stressors as a team (i.e. responses were not different than the baseline). There was, however, a clear increase for all cytokine levels (statistically significant $(\mathrm{p}<0.05)$ for 7 out of 9 cytokines) due to experienced failure when the subjects completed the tasks as individuals. These results not only suggest the involvement of the inflammatory and immune system in the human response to stress experienced with attempt and failure, but also implies the distribution of both negative and positive stress among multiple individuals. Since a large contributor of cytokine production comes from a physical aspect (studies demonstrating increased cytokine levels resulting from exercise [35], it can be argued that cytokine levels did not change from the baseline when subjects worked in teams because the physical efforts were divided among 3-4 people. However, Table 4.2 shows that on average the subjects spent more time completing the tasks as a team, requiring extra effort for a longer duration, but cytokine levels did not significantly increase during team performances $(\mathrm{p}>0.05)$. In addition, there were no substantial differences between the average times taken to complete the successful and failed tasks as individuals ( 6 min $22 \mathrm{sec}$ vs $7 \mathrm{~min} 1 \mathrm{sec}$ ), but there is a clear increase in cytokine production after 
failed tasks. These results suggest that the increases in cytokine production are due to the stress of experienced failure rather than physical exertion.

The variation within the subject population must also be considered. It is highly unlikely for all subjects to react in the same way, let alone produce the same cytokine response. This resulted in the notable error bars seen in Figure 1. However, even with the variation among the subjects, there were still statistically significant differences between cytokine responses resulting from success and failure $(\mathrm{p}<0.05)$. Nonetheless, there were still noticeable changes in the cytokine profile among all 14 subjects, as seen in Figures B1-B4 in Appendix B. In those figures, cytokine concentrations following successful and failed attempts for both individual and team efforts are again normalized to the baseline for all subjects. There was quite a range of responses, making it challenging to find a pattern or even group together subjects who had similar responses. There were many things to consider: differences in cytokine levels associated with success and failure during individual tasks, success and failure during team tasks, success during both individual and team tasks, failure during both individual and team tasks, and the magnitude of the response. Some subjects, like subject 6 , exhibited a clear increase in overall cytokine production after experiencing individual failure, whereas subject 3 had elevated responses following team success. Then, the cytokine profile of some subjects (subject 12) was not considerably affected by success, failure, individual work, or team work, while other subjects (subject 13) had responses that were more than 5-10 times higher than the baseline. In addition, various cytokines presented different patterns, further complicating the efforts to decipher the biochemical response. As a result, principal component analysis (PCA) and hierarchal clustering were employed. 
Both PCA and clustering results (Figures $4.2-4.5$ ) revealed tight groupings and separations of cytokine responses associated with success and failure, as measured following individual and team performances. These results further demonstrate that even with subject variation as a factor, properly analyzed salivary cytokines can capture the stress response. However, 3 cytokines (IL-1 $\beta$, IL-6, TNF- $\alpha$ ) appeared to be mis-clustered, suggesting that these cytokines are not informative in distinguishing the different stressors in this specific case. This may be attributed to the fact that overall, the normalized responses of these cytokines were not as amplified as the others or have more complex roles due to their broad effects [36]. It is essential to note that the significance or insignificance of certain cytokines is case specific and IL-1 $\beta$, IL-6, TNF- $\alpha$ may be central in the reaction to stress, as indicated in previous studies [37, 38].

For this study, all responses were normalized to baseline levels as individuals produce varied basal salivary cytokines and this investigation focused on the cytokine alterations due to stress. Interestingly, baseline cytokine levels presented insightful results regarding stress driven by social stress or anticipation. Overall, baseline levels before team experiments were comparatively higher than baseline levels before individual experiments, as shown in Table 4.3. This finding raises an important concept of the social stress of performing with other individuals that can impact cytokine production. Social stressors have previously been demonstrated to elicit cytokine response [39] and this study further establishes cytokine roles in the context of social stress fueled by the idea of working with other individuals, or performing in front of them. While only 4 responses (IL-1 $1 \beta$, IL-6, TNF- $\alpha$, and GM-CSF) were significantly different $(p<0.05)$ between individual and team baselines, all cytokines followed the general trend. Earlier, IL-1 $\beta$, IL-6, TNF- $\alpha$ were mentioned to not be contributive in describing the stress response, but perhaps 
these cytokines were already elevated from the beginning and continued to stay raised throughout the rest of the study.

Cortisol concentrations were also observed as an additional measurement of experienced stress (Table 4.4). Cortisol is well known as a biomarker used to understand the effects of stress [40], as well as monitoring the results of stress mediation [41]. Salivary cortisol levels have been observed to increase as a result of acute stress, chronic stress [42], and even social stress [43]. In this study, cortisol concentrations were found to be overall decreased following team performances, but there were no significant differences associated with experienced success and failure. While cortisol measurements could not be used to distinguish between positive and negative stress like cytokine responses, it does follow the trend and supports the concept that stress may be distributed among individuals.

While this work offers exciting information elucidating the cytokine response to stress, more research is needed to fully understand how cytokines can be used as salivary biomarkers. There are many factors that can interfere with cytokine production that should be investigated. First, it is always a challenge to identify all potential stressors that can alter biochemical processes. This particular study involved potential physical, psychological, social, positive, negative, and internal stressors, to name a few. Numerous stressors can make it difficult to pinpoint what is responsible for the observed changes. Additionally, not all subjects experienced the same extent of stress. For instance, a smaller subject will need to exert more effort to lift a 70 pound punching bag, compared to a larger subject. Timing of sample collection is another imperative variable in identifying relevant biomarkers [44] and additional samples collected post-stressor (not just 
immediately after) will help track long-term fluctuations that may be relevant to the overall response. Finally, there are a number of other cytokines that can be measured (e.g. chemokines, additional interferon, interleukin, and tumor necrosis factor families), that may contribute pertinent information to this work and could be investigated in the future.

\subsection{Conclusion}

This study offers insight into the complex inflammation and immune mechanisms involved in human stress response. By monitoring salivary cytokine production immediately following exposure to various stressors, certain patterns that can distinguish between positive and negative stress have been identified. Specifically, cytokine responses associated with experienced success and failure were captured. Even with the challenges of biochemical variation within the subject population, statistical analyses demonstrated the capacity of salivary cytokines to reflect adaptations in stress responses. Furthermore, the findings of this work explored the concept that stress can be distributed among multiple individuals.

\subsection{Acknowledgements}

This research was conducted by support from Defense Advanced Research Projects Agency (DARPA) [N66001-09-1-2090-WVA]; U. S. Army Research Laboratory and the U. S. Army Research Office [W911NF-12-1-0165]; and West Virginia University. 


\subsection{References}

[1] Streckfus CF, Bigler LR. Saliva as a diagnostic fluid. Oral Dis. 2002;8(2):69-76.

[2] Sahibzada HA, Khurshid Z, Khan RS, Naseem M, Siddique KM, Mali M, et al. Salivary IL-8, IL-6 and TNF-alpha as Potential Diagnostic Biomarkers for Oral Cancer. Diagnostics (Basel). $2017 ; 7(2)$.

[3] Soni S, Agrawal P, Kumar N, Mittal G, Nishad DK, Chaudhury NK, et al. Salivary biochemical markers as potential acute toxicity parameters for acute radiation injury: A study on small experimental animals. Hum Exp Toxicol. 2016;35(3):221-8.

[4] Szabo YZ, Newton TL, Miller JJ, Lyle KB, Fernandez-Botran R. Acute stress induces increases in salivary IL-10 levels. Stress. 2016;19(5):499-505.

[5] Malamud D. Saliva as a diagnostic fluid. Dent Clin North Am. 2011;55(1):159-78.

[6] Humphrey SP, Williamson RT. A review of saliva: normal composition, flow, and function. J Prosthet Dent. 2001;85(2):162-9.

[7] Garrett JR. The proper role of nerves in salivary secretion: a review. J Dent Res. 1987;66(2):387-97.

[8] Carpenter GH. The secretion, components, and properties of saliva. Annu Rev Food Sci Technol. 2013;4:267-76.

[9] Proctor GB, Carpenter GH. Regulation of salivary gland function by autonomic nerves. Auton Neurosci. 2007;133(1):3-18. 
[10] Zvezdanović L, Đorđević V, Ćosić V, Cvetković T, Kundalić S, Stanković A. The significance of cytokines in diagnosis of autoimmune diseases. Jugoslovenska medicinska biohemija. 2006;25(4):363-72.

[11] Aziz S, Ahmed SS, Ali A, Khan FA, Zulfiqar G, Iqbal J, et al. Salivary Immunosuppressive Cytokines IL-10 and IL-13 Are Significantly Elevated in Oral Squamous Cell Carcinoma Patients. Cancer Invest. 2015;33(7):318-28.

[12] Javed F, Al-Kheraif AA, Al Amri MD, Alshehri M, Vohra F, Al-Askar M, et al. Periodontal Status and Whole Salivary Cytokine Profile Among Smokers and Never-Smokers With and Without Prediabetes. J Periodontol. 2015;86(7):890-8.

[13] Thorman R, Lundahl J, Yucel-Lindberg T, Hylander B. Inflammatory cytokines in saliva: early signs of metabolic disorders in chronic kidney disease. A controlled cross-sectional study. Oral Surg Oral Med Oral Pathol Oral Radiol Endod. 2010;110(5):597-604.

[14] King GL. The role of inflammatory cytokines in diabetes and its complications. J Periodontol. 2008;79(8 Suppl):1527-34.

[15] Lim PW, Garssen J, Sandalova E. Potential Use of Salivary Markers for Longitudinal Monitoring of Inflammatory Immune Responses to Vaccination. Mediators of inflammation. 2016 Feb 28;2016.

[16] Roescher N, Tak PP, Illei GG. Cytokines in Sjögren's syndrome. Oral Dis. 2009;15(8):51926.

[17] Slavish DC, Graham-Engeland JE, Smyth JM, Engeland CG. Salivary markers of inflammation in response to acute stress. Brain Behav Immun. 2015;44:253-69. 
[18] Riis JL, Granger DA, DiPietro JA, Bandeen-Roche K, Johnson SB. Salivary cytokines as a minimally-invasive measure of immune functioning in young children: correlates of individual differences and sensitivity to laboratory stress. Dev Psychobiol. 2015;57(2):153-67.

[19] Dantzer R, O’Connor JC, Freund GG, Johnson RW, Kelley KW. From inflammation to sickness and depression: when the immune system subjugates the brain. Nat Rev Neurosci. 2008;9(1):46-56.

[20] Allen AP, Kennedy PJ, Cryan JF, Dinan TG, Clarke G. Biological and psychological markers of stress in humans: focus on the Trier Social Stress Test. Neurosci Biobehav Rev. 2014;38:94124.

[21] Ng V, Koh D, Chan G, Ong HY, Chia SE, Ong CN. Are salivary immunoglobulin A and lysozyme biomarkers of stress among nurses? J Occup Environ Med. 1999;41(10):920-7.

[22] Masilamani R, Darus A, Ting AS, Ali R, Mahmud AB, David K. Salivary biomarkers of stress among teachers in an urban setting. Asia Pac J Public Health. 2012;24(2):278-87.

[23] Juster RP, McEwen BS, Lupien SJ. Allostatic load biomarkers of chronic stress and impact on health and cognition. Neurosci Biobehav Rev. 2010;35(1):2-16.

[24] Lehner P, Seyed-Solorforough MM, O'Connor MF, Sak S, Mullin T. Cognitive biases and time stress in team decision making. IEEE Transactions on Systems, Man, and Cybernetics-Part A: Systems and Humans. 1997 Sep;27(5):698-703.

[25] Ellis AP. System breakdown: The role of mental models and transactive memory in the relationship between acute stress and team performance. Academy of Management Journal. 2006 Jun 1;49(3):576-89. 
[26] Driskell JE, Salas E. Group decision making under stress. Journal of Applied Psychology. 1991 Jun;76(3):473.

[27] Shields GS, Kuchenbecker SY, Pressman SD, Sumida KD, Slavich GM. Better cognitive control of emotional information is associated with reduced pro-inflammatory cytokine reactivity to emotional stress. Stress. 2016;19, 63-68.

[28] Walsh E, Eisenlohr-Moul T, Baer R.. Brief mindfulness training reduces salivary IL-6 and TNF-alpha in young women with depressive symptomatology. J Consult Clin Psychol. 2016;84, 887-897.

[29] Izawa S, Sugaya N, Kimura K, Ogawa N, Yamada KC, Shirotsuki K, et al. An increase in salivary interleukin-6 level following acute psychosocial stress and its biological correlates in healthy young adults. Biol Psychol. 2013;94(2):249-54.

[30] Newton TL, Fernandez-Botran R, Lyle KB, Szabo YZ, Miller JJ, Warnecke AJ. Salivary Cytokine Response in the Aftermath of Stress: An Emotion Regulation Perspective. Emotion. 2017.

[31] Jaedicke KM, Preshaw PM, Taylor JJ. Salivary cytokines as biomarkers of periodontal diseases. Periodontol 2000. 2016;70(1):164-83.

[32] Korostoff A, Reder L, Masood R, Sinha UK. The role of salivary cytokine biomarkers in tongue cancer invasion and mortality. Oral Oncol. 2011;47(4):282-7.

[33] Mastrolonardo M, Alicino D, Zefferino R, Pasquini P, Picardi A. Effect of psychological stress on salivary interleukin-1beta in psoriasis. Arch Med Res. 2007;38(2):206-11. 
[34] Siqueira EC, de Souza FT, Ferreira E, Souza RP, Macedo SC, Friedman E, et al. Cell phone use is associated with an inflammatory cytokine profile of parotid gland saliva. J Oral Pathol Med. 2016;45(9):682-6.

[35] Pedersen BK, Bruunsgaard H, Ostrowski K, Krabbe K, Hansen H, Krzywkowski K, et al. Cytokines in aging and exercise. Int J Sports Med. 2000;21 Suppl 1:S4-9.

[36] Akira, S., Hirano, T., Taga, T., Kishimoto, T. Biology of multifunctional cytokines: IL 6 and related molecules (IL 1 and TNF). Faseb j. 1990;4:2860-2867.

[37] Gill J, Vythilingam M, Page GG. Low cortisol, high DHEA, and high levels of stimulated TNF-alpha, and IL-6 in women with PTSD. J Trauma Stress. 2008;21(6):530-9.

[38] Zefferino R, Facciorusso A, Lasalvia M, Narciso M, Nuzzaco A, Lucchini R, et al. Salivary markers of work stress in an emergency team of urban police (1 degree step). G Ital Med Lav Ergon. 2006;28(4):472-7.

[39] Audet MC, McQuaid RJ, Merali Z, Anisman H. Cytokine variations and mood disorders: influence of social stressors and social support. Front Neurosci. 2014;8.

[40] Hellhammer DH, Wust S, Kudielka BM. Salivary cortisol as a biomarker in stress research. Psychoneuroendocrinology. 2009;34(2):163-71.

[41] Yoo YG, Lee DJ, Lee IS, Shin N, Park JY, Yoon MR, et al. The Effects of Mind Subtraction Meditation on Depression, Social Anxiety, Aggression, and Salivary Cortisol Levels of Elementary School Children in South Korea. J Pediatr Nurs. 2016;31(3):e185-97. 
[42] Gonzalez-Cabrera J, Fernandez-Prada M, Iribar-Ibabe C, Peinado JM. Acute and chronic stress increase salivary cortisol: a study in the real-life setting of a national examination undertaken by medical graduates. Stress. 2014;17(2):149-56.

[43] Takahashi T, Ikeda K, Ishikawa M, Kitamura N, Tsukasaki T, Nakama D, et al. Anxiety, reactivity, and social stress-induced cortisol elevation in humans. Neuro Endocrinol Lett. 2005;26(4):351-4.

[44] Reed RG, O'Connor MF, Pace TW, Raison CL, Butler EA. Dyadic coping and salivary interleukin-6 responses to interpersonal stress. J Fam Psychol. 2017;31(3):367-73. 


\section{Chapter 5}

Identification of Biomarkers Related to Perceived Stress Scale10 and the Evaluation of the Survey Components 


\section{Identification of biomarkers related to Perceived Stress Scale-10 and the evaluation of the survey components ${ }^{2}$}

Perceived stress surveys have been used by health specialists for decades to provide quick assessments of the degree of stress during a specified time, and have even been shown to be predictive of response to stressors or treatment outcomes. While these surveys have become an essential tool to evaluate the psychological state of individuals as a result of traumatic events or health concerns, very little research has related known biomarkers of stress to survey results. Identification of biomarkers associated with perceived stress scale scores, and even specific survey questions, provides important supplemental information for both practitioners and researchers using these surveys, and could offer a means to enhance interpretation of survey responses from a biological perspective. In this study, subjects completed a commonly accepted PSS-10 (Perceived Stress Scale-10) survey and provided corresponding blood samples. To identify biomarkers associated with perceived stress scores, plasma was analyzed for a variety of biomarkers. Adrenocorticotropic hormone $(\mathrm{ACTH})$ was identified as a key biomarker, with concentrations significantly different between low and high perceived stress subjects. The ten questions of the PSS-10 were further dissected to understand the degree of contribution each question offers to the overall stress assessment. ACTH again, along with cortisol and insulin were observed to be associated with scores of individual questions, highlighting certain questions as the driving forces of the overall score. An understanding of the impact each individual question possesses may be essential to acquiring an accurate assessment of perceived stress.

\footnotetext{
${ }^{2}$ Parts of this chapter have been published previously from Han AA, Vrana JA, Mitchell C, Boyd JW. Identification of Biomarkers Related to Perceived Stress Scale-10 and the Evaluation of the Survey Components. J Med Genomics Biomark. 2015;2(003). Reproduced with permission from Herald Scholarly Open Access.
} 


\subsection{Introduction}

Dynamic physiological, behavioral, and biochemical changes are anticipated outcomes of stress, triggered by external demands overwhelming an individual's ability to cope [1]. The degree of stress can have a considerable negative or positive impact on performance [2], mood [3], and even health [4]. Evidence suggesting stress levels may prompt or alter biochemical responses [5], and even epigenetic mechanisms [6], offers a link between stress and the progression of mental or physical diseases. Monitoring stress levels of patients may be an important component of treatment or recovery, and stress surveys are one of the frequently used methods to make such assessments. Hospital Anxiety and Depression Scale (HADS) [7], Perceived Stress Scale (PSS) [8], State Trait Anxiety Inventory (STAI) [9], and General Health Questionnaire (GHQ) [10] are several notable stress assessment tools designed to extract and assemble pertinent information indicative of stress.

While stress scales are informative, there are limitations in the various ways questions may be interpreted, emphasizing the importance of appropriately selected and constructed questions [11]. Inevitable and uncontrollable factors, such as the mood and condition of the subject, may additionally influence the manner by which questions are read and answered [12]. As such, further investigation of biomarkers related to perceived stress scales provides insight regarding the accuracy and reliability of perceived stress scales. Well known biomarkers of stress, such as cortisol [13], cytokines [14], and catecholamines [15] have been reported to be correlated to scores of perceived stress [16], and potentially even predictive of varying degrees of stress and stressrelated outcomes [17].

The PSS-10 (Perceived Stress Scale-10) is a specific stress survey which provides a means to measure self-evaluated stress [18], and is a widely employed tool that has been used to assess 
the psychological impact contributing to experienced stress [19]. The questions of the PSS-10 are formed to assess stress levels accumulated in the last month. Therefore, significant biomarkers detected in blood samples are assumed to result from a collection of pre-existing stress instead of a single stressful event. Perceived stress scales serve to quickly appraise the degree of stress under demanding and traumatic conditions [20,21], gauge the effectiveness of treatments aimed to reduce stress [22], and investigate the relationship between the level of stress and behavioral patterns or health disorders $[23,24]$. In the present study, the PSS-10 survey is examined in whole, but also dissected in order to identify unique biomarkers related to each question individually. Comprised of 10 questions (See PSS-10 questions in Table 5.1), the survey is designed to incorporate both positive and negative approaches of evaluation. Factors such as the ability to maintain control over stressful events, along with the positive and negative emotions involved in coping with stress are addressed in the PSS-10. Questions 4, 5, 7, and 8 address positive factors anticipated to reduce perceived stress, while questions $1,2,3,6,9$, and 10 target negative aspects contributing to stress. An output of numerical scores between zero and 40 allows for simple comparison or differentiation among subjects participating in the same study. Examination of individual questions of the survey may indicate which types of questions offer significant contribution toward generating the overall PSS-10 score.

This study includes a straightforward assessment of PSS-10 scores and blood samples acquired, with an intention to discern biomarkers related to PSS-10 results. The intent of this study is not to ascertain biomarkers after a controlled exposure to stressors, but to identify and examine biomarkers related to self-perceived stress. Over 20 endogenous biomarkers, including cortisol, insulin, adrenocorticotropic hormone, testosterone, epinephrine, and cytokines were selected for 
this study. A full list of the biomarkers is detailed in the Discussion section. This selection of biomarkers focuses on general indicators related to stress and the response to stress (ie cortisol [25], ACTH [26], epinephrine and norepinephrine [27]), as well as key regulators of basic cellular activities (ie glucose, insulin, glutathione, adenosine triphosphate, chloride). Considerably low or high levels of these biomarkers may describe the degree of stress [28].

\subsection{Materials and methods}

\subsubsection{Subjects}

Students from the Air Force Reserve Officers' Training Corps at West Virginia University were recruited to participate in this study. A total of 17 healthy subjects (15 male, 1 female), between the ages of 18-22, were enrolled. One male subject was excluded from analysis due to insufficient blood sample. Additionally, a female subject was excluded because we were only able to recruit one female. The protocol for this study was approved by the Institutional Review Board at West Virginia University and USAMRMC Office of Research Protections. All subjects provided written and informed consent to be willing test subjects prior to proceeding with the study. A detailed profile (Age, body fat percentage, exercise, tobacco and alcohol use, caffeine and food intake, and medication use) of each subject is included in Table 5.2. 


\subsubsection{Perceived Stress Scale (PSS-10)}

The subjects were directed to answer 10 questions pertaining to stress appraisal upon arrival to the research site. Overall perceived stress scores based on the questions of the survey and individual scores for each question were determined per published direction [18]. All questions were provided with possible answer choices of a 5-level Likert type scale (never, almost never, sometimes, fairly often, very often). Each answer was assigned a number rating (0-4) and summed up for all 10 questions to acquire the overall score. The number ratings for positively stated questions (items 4, 5, 7, \& 8) were reversed.

Table 5.1. 10 questions of the PSS-10 survey supplied to the subjects. Italicized questions (Q4, 5, 7 , and 8 ) are positively phrased.

\section{Question}

In the last month, how often have you been upset because of something that happened unexpectedly?

In the last month, how often have you felt that you were unable to control the important things in your life?

In the last month, how often have you felt nervous and "stressed"?

In the last month, how often have you felt confident about your ability to handle your personal problems?

In the last month, how often have you felt that things were going your way?

In the last month, how often have you found that you could not cope with all the things that you had to do?

In the last month, how often have you been able to control irritations in your life?

In the last month, how often have you felt that you were on top of things?

In the last month, how often have you been angered because of things that were outside your control?

In the last month, how often have you felt difficulties were piling up so high that you could not overcome them? 
Table 5.2. Profiles of each subject

\begin{tabular}{|c|c|c|c|c|c|c|c|c|}
\hline Subject & Age & $\begin{array}{l}\text { Body Fat } \\
\%\end{array}$ & $\begin{array}{c}\text { Exercise } \\
\text { (days per } \\
\text { week) }\end{array}$ & Tobacco & Alcohol (how often) & $\begin{array}{l}\text { Caffeinated beverage } \\
\text { (when, how much) }\end{array}$ & $\begin{array}{c}\text { Time since } \\
\text { last meal } \\
\text { (hours) }\end{array}$ & Medication \\
\hline 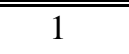 & 22 & 13.5 & $6--7$ & No & Yes (weekends) & Lunch, 12 oz Coke & 2.5 & NA \\
\hline 2 & 20 & 10.2 & $4--5$ & Yes & Yes (1-2 times a week) & 6 days ago, 1 can & 0.5 & $\begin{array}{l}\text { Ibuprofen, Sudafed, Mucinex } \\
\text { (none in last } 4 \text { days) }\end{array}$ \\
\hline 3 & 19 & 20.9 & $2--3$ & No & No & 2 days ago, 1 soda can & 1.0 & NA \\
\hline 4 & 19 & 6.1 & $2--3$ & No & Yes (once a month) & Months ago & 4.0 & NA \\
\hline 5 & 19 & 14.4 & $4--5$ & No & No & NA & 0.75 & NA \\
\hline 6 & 19 & 6.2 & $6--7$ & No & $\begin{array}{l}\text { Yes (once every couple } \\
\text { weeks) }\end{array}$ & A week ago, cup of coffee & 2.0 & NA \\
\hline 7 & 20 & 15.9 & $6--7$ & No & Yes (occasionally) & 10:30 AM, cup of coffee & 1.0 & NA \\
\hline 8 & 19 & 12.5 & $6--7$ & Yes & Yes (2-4 times a month) & Morning, 6 oz coffee & 1.5 & $\begin{array}{l}\text { Vitamin C supplement, } \\
\text { Ibuprofen, Melatonin }\end{array}$ \\
\hline 9 & 18 & 17.9 & $2--3$ & No & Yes (Rarely) & Earlier that day, cup of tea & 5.0 & NA \\
\hline 10 & 20 & 13.7 & $6--7$ & No & No & Month ago, cup of coffee & 2.0 & NA \\
\hline 11 & 19 & 17.2 & $4--5$ & No & No & $\begin{array}{l}\text { 1-2 weeks ago, cup of } \\
\text { coffee }\end{array}$ & 5.5 & NA \\
\hline 12 & 18 & 22.9 & $2--3$ & No & Yes (Once a week) & 10:40 AM, 2 glasses of tea & 0.67 & Multivitamin, Advil \\
\hline 13 & 19 & 15.8 & $4--5$ & No & No & 1 hour, 4 fl. Oz & 1.0 & NA \\
\hline 14 & 19 & 14 & $6--7$ & No & Yes (not often) & Morning, 20 fl. Oz coke & 1.0 & NA \\
\hline 15 & 21 & 21 & $4--5$ & Yes & Yes (5 nights a week) & 30 min, 1 can of coke & 2.0 & NA \\
\hline
\end{tabular}




\subsubsection{Plasma sample preparation and analysis}

Approximately one hour after the subjects completed the PSS-10 (between 8 and 8:30 PM), blood samples were acquired. A butterfly needle was used to draw blood samples into BD Vacutainer plasma preparation tubes (BD, Franklin Lakes, NJ) or BD Vacutainer plastic blood collection tubes coated with $\mathrm{K}_{2}$ EDTA (BD, Franklin Lakes, NJ). Immediately after collection of the blood, the tubes were inverted 10 times, followed by centrifugation at 2500 RPM for 12 minutes to form the top plasma layer. The samples were stored in ice until they were transported to a BSL-2 lab at West Virginia University. To avoid freeze/thaw cycles, $200 \mu \mathrm{L}$ aliquots of the plasma layer were removed from the blood tube and stored in cryogenic vials at $-80^{\circ} \mathrm{C}$. An ELISA kit from ALPCO (Salem, NH, Catalog \# 21-ACTHU-E01) was purchased to quantify ACTH. The intra-assay and inter-assay coefficients of variance (CV) for ACTH were less than 7\% and 8\%, respectively. Insulin was measured using a custom designed Milliplex kit obtained from EMD Millipore (Billerica, MA, Catalog \# HMHMAG34K). The intra-assay and inter-assay CV for insulin were less than $16 \%$ and $25 \%$ respectively. Cortisol was assayed with an EIA kit from Cayman Chemical (Ann Arbor, MI, Catalog \# 500360). The intra-assay and inter-assay CV for cortisol were less than $14 \%$ and $26 \%$, respectively.

\subsubsection{Statistical analysis}

Maximum and minimum scores for the overall survey were 40 and zero, respectively, and four and zero for each individual question. Subjects with overall scores between zero and ten were grouped as low stress subjects and those with overall scores above 10 were identified as highstressed subjects. Subjects with scores of 0 and 1 on individual questions were grouped as low stressed and those with scores above 2 were assigned to the high stressed group. Levels of relevant 
biomarkers found in initial samples of plasma were compared between the low stress and high stress groups via Welch-corrected t-tests using Prism V5 (Graphpad Software, San Diego, CA). Outliers were removed accordingly by the use of a Grubbs' test.

\subsection{Results}

\subsubsection{Biomarkers of overall PSS-10 scores}

Analysis of overall PSS-10 scores in relation to biomarkers found in samples of plasma, highlighted ACTH as a potential indicator of perceived stress levels, as levels of plasma ACTH were significantly decreased in subjects with lower overall PSS-10 scores. Mean ACTH concentrations of subjects in low and high perceived stress score groups were $18.4 \pm 1.3 \mathrm{pg} / \mathrm{mL}$ $(\mathrm{N}=6)$ and $27.3 \pm 3.2 \mathrm{pg} / \mathrm{mL}(\mathrm{N}=9)$, respectively, $\mathrm{p}<0.05$ (Figure 5.1).

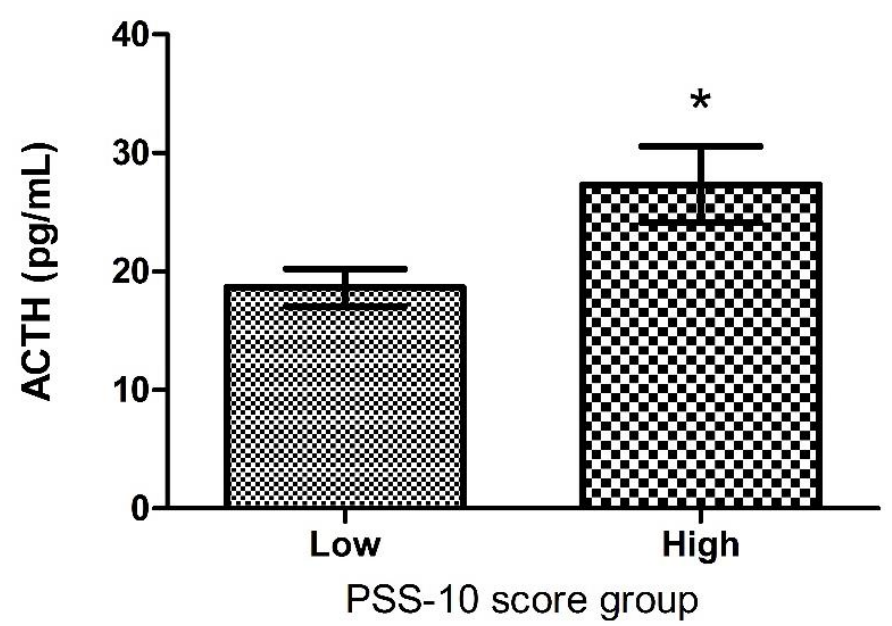

Figure 5.1. Plasma ACTH levels of high and low perceived stress subjects. Mean plasma Adrenocorticotropic Hormone (ACTH) levels of subjects with overall low and high perceived stress scores were analyzed. Observed mean ACTH values found to be significantly different $(\mathrm{p}<$ 0.05 ) between high and low perceived stress groups are marked with *. Error bars reflect \pm standard error of the mean. 


\subsubsection{Biomarkers of PSS-10 question 2 (Q2) scores}

Additional examination of each individual question comprising the PSS-10 revealed perceived stress scores of select questions were considerably related to levels of certain plasma biomarkers. In specific, plasma cortisol was identified to be distinct between the group of subjects with higher scores for question $2(\mathrm{Q} 2)$ and the group with lower scores. Mean cortisol concentrations of subjects in low and high perceived stress groups were $61.3 \pm 8.8 \mathrm{pg} / \mathrm{mL}(\mathrm{N}=9)$ and $35.8 \pm 6.4 \mathrm{pg} / \mathrm{mL}(\mathrm{N}=6)$, respectively, $\mathrm{p}<0.05$ (Figure 5.2).

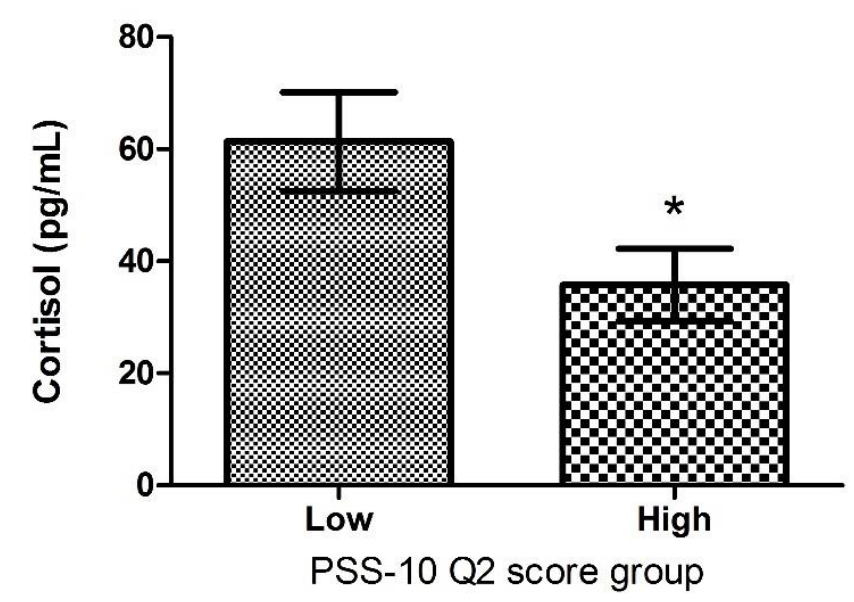

Figure 5.2. Plasma cortisol levels of high and low perceived stress subjects - Q2. Mean plasma cortisol levels of subjects with low and high perceived stress scores were determined for Q2. Observed mean cortisol values found to be significantly different $(\mathrm{p}<0.05)$ between low and high perceived stress groups are marked with *. Error bars reflect \pm standard error of the mean.

\subsubsection{Biomarkers of PSS-10 question 5 (Q5) scores}

Scores of Q5 were also associated with concentrations of plasma ACTH. Subjects placed in the low perceived stress group, according to Q5 results, had lower concentrations of ACTH in plasma samples. Mean ACTH concentrations of subjects in low and high perceived stress score groups were $18.9 \pm 1.5 \mathrm{pg} / \mathrm{mL}(\mathrm{N}=10)$ and $33.5 \pm 3.0 \mathrm{pg} / \mathrm{mL}(\mathrm{N}=5)$, respectively, $\mathrm{p}<0.05$. (Figure 5.3). 


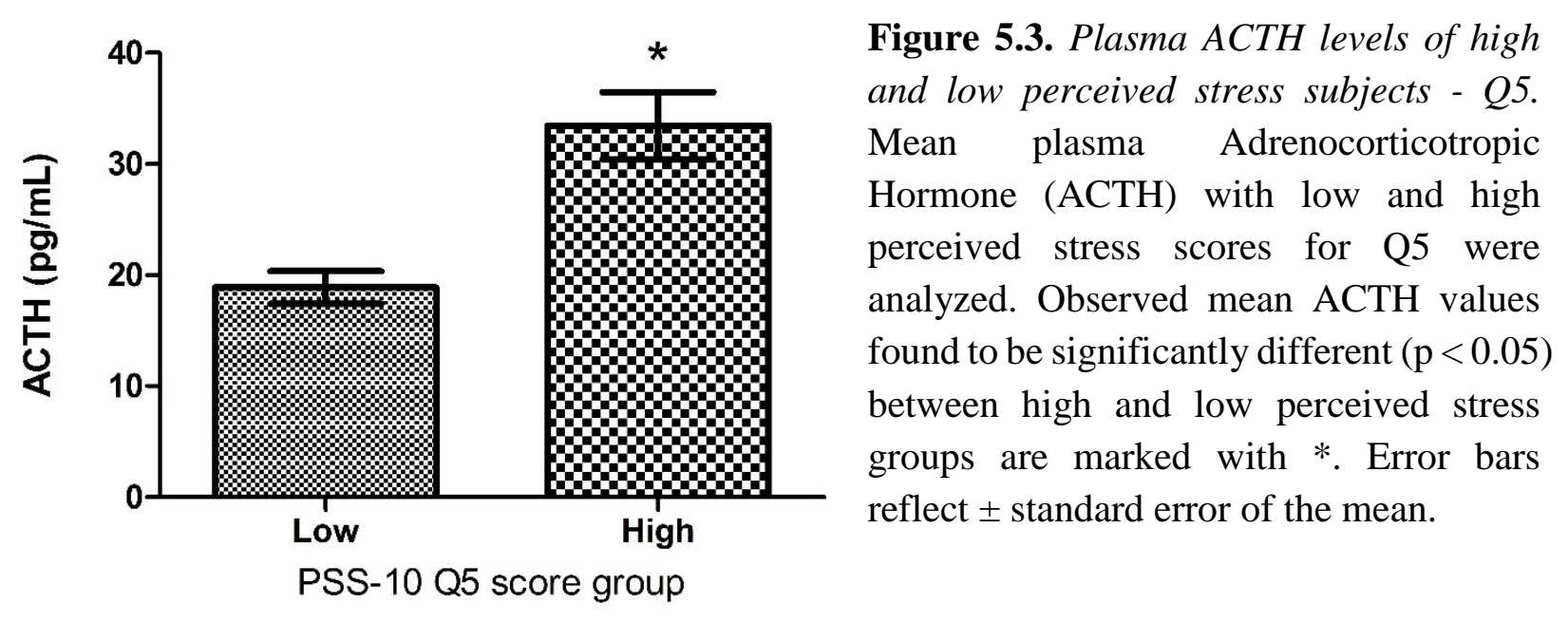

\subsubsection{Biomarkers of PSS-10 question 7 (Q7) scores}

Scores of question 7 were related to concentrations of plasma insulin. Higher plasma insulin levels in low perceived stress groups, classified based on questions 7 scores, were observed. Mean insulin concentrations of subjects in low and high perceived stress score groups, assembled based on question 7 scores, were $792.7 \pm 92.9 \mathrm{pg} / \mathrm{mL}(\mathrm{N}=10)$ and $344.7 \pm 37.9 \mathrm{pg} / \mathrm{mL}(\mathrm{N}=5)$, respectively, $\mathrm{p}<0.05$ (Figure 5.4).

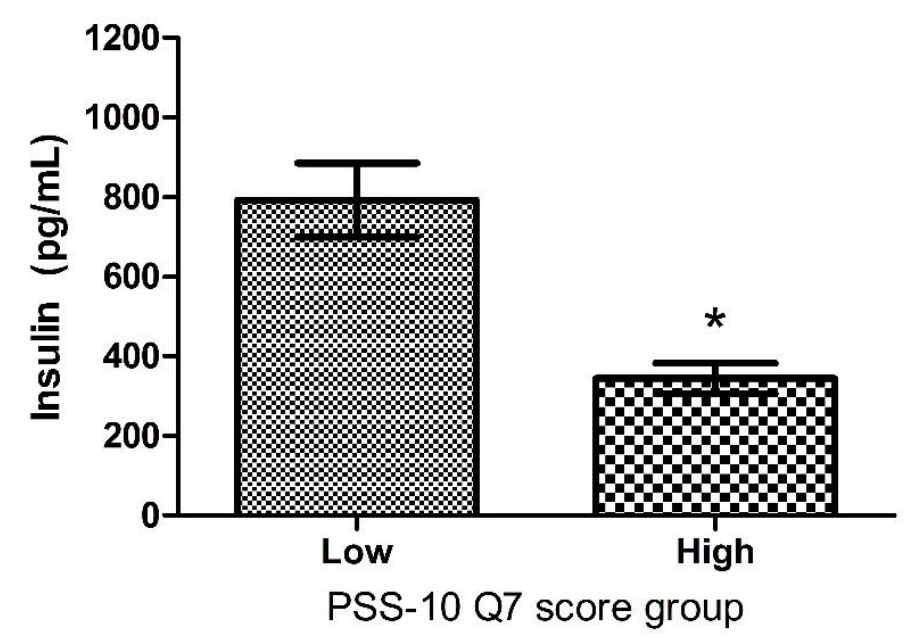

Figure 5.4. Plasma insulin levels of high and low perceived stress subjects - Q7. Mean plasma insulin levels of subjects with low and high perceived stress scores for Q7 were determined. Observed mean insulin values found to be significantly different $(p<0.05)$ between high and low perceived stress groups are marked with *. Error bars reflect \pm standard error of the mean. 


\subsubsection{Biomarkers of PSS-10 question 9 (Q9) scores}

Distinct insulin levels described low and high perceived stress groups, generated based on question 9 scores. Mean insulin concentrations of $798.9 \pm 94.8 \mathrm{pg} / \mathrm{mL}(\mathrm{N}=11)$ and $383.7 \pm 51.8$ $\mathrm{pg} / \mathrm{mL}(\mathrm{N}=5)$, were determined for low and high scoring groups, respectively, $\mathrm{p}<0.05$ (Figure $5.5)$.

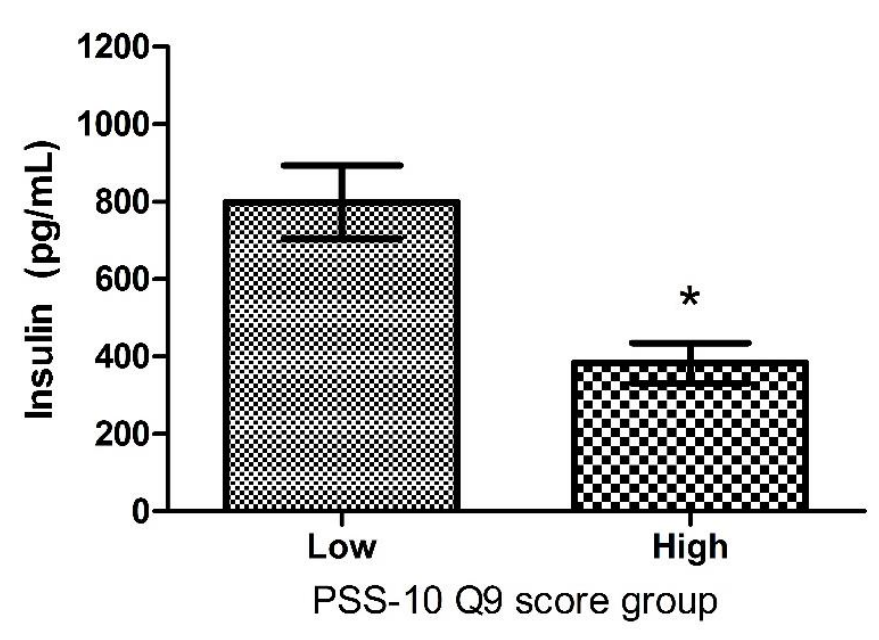

Figure 5.5. Plasma insulin levels of high and low perceived stress subjects - $Q 9$. Mean plasma insulin levels of subjects with low and high perceived stress scores for Q9 were analyzed. Observed mean insulin values found to be significantly different $(\mathrm{p}<0.05)$ between high and low perceived stress groups are marked with *. Error bars reflect \pm standard error of the mean.

\subsection{Discussion}

As informative as these stress surveys may be, there is always a possibility of misrepresentation or misinterpretation. Identifying plasma biomarkers that are significantly different between subjects of low and high perceived stress may be a suitable and quantitative alternative, or supplement, to assessing perceived stress. In this study, various stress-related biomarkers (ACTH, cortisol, insulin, glucose, leptin, testosterone, cytokines [IL-1B, IL-10, IL-6, GM-CSF, IL-5, IFN- $\gamma$, TNF- $\alpha$, IL-2, and IL-4], epinephrine, norepinephrine, adenosine triphosphate, aspartate aminotransferase, creatine kinase, chloride, and glutathione) were 
measured. Of the biomarkers measured, ACTH was identified to exhibit significantly distinct concentrations in plasma of subjects belonging in low and high perceived stress groups (Figure 5.1). ACTH levels generally increase upon exposure to stressors $[29,30]$ and have been linked to perceived stress [31]. In accordance with these previous findings associating ACTH with stress, subjects with higher perceived stress had significantly elevated concentrations of ACTH in this study. Although ACTH was the only statistically significant biomarker related to overall PSS-10 scores, further analysis of each individual question uncovered several other biomarkers.

The purpose of dissecting the survey and analyzing each question separately was to understand how and to what extent each question contributes to the overall stress assessment. Upon analysis of the individual questions, ACTH, cortisol, and insulin were found to be associated with several questions. The group of subjects that scored higher for Q5 had significantly greater levels of plasma ACTH (Figure 5.3). As ACTH concentrations were related to overall PSS-10 scores, a similar outcome between ACTH levels and individual question scores was also expected. These results suggest how a participant answers Q5 may be particularly important to assessing overall perceived stress. Along with Q5, three other questions (Q2, Q7, and Q9), offered insight regarding the connection between the survey results and related biomarkers. Q2 scores were related to plasma cortisol (Figure 5.2), while Q7 and Q9 scores were associated with plasma insulin levels (Figures 5.4 and 5.5). Cortisol and insulin were only significantly significant when analyzed against individual questions, suggesting the importance of analyzing the results of individual question in conjunction with overall scores. 
As a proposed hallmark of stress, cortisol is often measured to determine the degree of stress after demanding and taxing events [25]. A general increase in cortisol levels is expected in those that are more stressed [32], but there is also great variability in cortisol secretion [33]. In this study, the group of subjects that scored higher for Q2 (i.e. exhibited more perceived stress) had a lower average plasma cortisol than the group that had lower scores. The data suggest that cortisol levels may be lower in subjects that perceive higher stress since this survey question assesses an accumulation of perceived stress over a prolonged period of one month, instead of after one specific incident. In a previous study, cortisol secretion was suppressed in subjects who had experienced stress over an extended duration of time [34].

Insulin was an unexpected biomarker as it is not a central stress-related hormone, but nonetheless, has been linked to stress [35]. In this study, subjects with lower PSS-10 scores had higher insulin levels, which suggests subjects with lower insulin levels actually experienced more stress. In fact, stress hormones are able to strongly regulate, and in some cases, inhibit the release of insulin [36]. A more apparent relationship between the survey results and measured insulin levels concerns when the subject had last eaten. An increase in plasma insulin concentration normally occurs after meals [37], and a correlation between the time of last food intake and insulin levels is expected to be negatively related. In agreement with the anticipated results, the plasma insulin levels in these subjects were significantly $(\mathrm{p}<0.05)$ and negatively correlated $(r=-0.6524)$ to the time since they had last eaten. Subjects that had recently eaten, scored lower on the overall PSS-10 (ie lower perceived stress), suggesting factors such as insulin concentration may affect how stress surveys are completed. While insulin is related to both Q7 and Q9 (one positive and one negative, respectively), it is unclear how positive and negative questions are distinctly related 
to a shared biomarker. In this study, we can only identify which questions and biomarkers are related, but to fully understand how biomarkers are related to individual questions, additional research is necessary.

There were a few limitations to this study. First, the sample size was not ideal, even though recruitment was attempted several times. Due to the small sample size, there were several questions $(\mathrm{Q} 3, \mathrm{Q} 4, \mathrm{Q} 8, \mathrm{Q} 10)$ that were not analyzed because there weren't definite low score and high score groups. For instance, for question 4 and 10, there was only one subject that belonged in the high score group, making it impossible to make any comparisons. With a bigger sample size, it may have been possible to analyze more individual questions and identify more biomarkers that may have been missed. The other biomarkers in our selection included glucose, leptin, testosterone, cytokines (IL-1 $\beta$, IL10, IL6, GM-CSF, IL5, IFN $\gamma$, TNF $\alpha$, IL2, and IL4), epinephrine, norepinephrine, adenosine triphosphate, aspartate aminotransferase, creatine kinase, chloride, and glutathione. While these biomarkers were not significant in our analysis, many of them are strongly related to stress. Due to the limitations of a small sample, it is feasible that anticipated biomarkers were statistically eliminated. Nonetheless, this study demonstrates a significance in identifying biomarkers to assess perceived stress, as well as a benefit in analyzing individual components of the survey. A bigger sample size and a wider selection of biomarkers will be necessary for future studies.

\subsection{Conclusion}

The findings of this study enabled us to identify key biomarkers associated with perceived stress and relate them to results determined from a commonly used and accepted survey. Analysis 
of these pertinent biomarkers then uncovered select questions that may be essential to the interpretation of perceived stress. An improved understanding of the perceived stress scale, from a biological perspective, may aid in the general assessment of stress and stress related outcomes.

\subsection{Acknowledgements}

This research was conducted by support from Defense Advanced Research Projects Agency (DARPA) [N66001-09-1-2090-WVA]; U. S. Army Research Laboratory and the U. S. Army Research Office [W911NF-12-1-0165]; and West Virginia University. We would like to acknowledge David Claypool for his assistance and expertise as a phlebotomist for this study.

\subsection{References}

[1] Cohen S, Kessler RC, Gordon LU (1995) Strategies for measuring stress in studies of psychiatric and physical disorders: Measuring stress: A guide for health and social scientists. Oxford University Press, New York.

[2] Hunziker S, Laschinger L, Portmann-Schwarz S, Semmer NK, Tschan F, et al. (2011) Perceived stress and team performance during a simulated resuscitation. Intensive Care Med 37: 1473-1479. http://link.springer.com/article/10.1007/s00134-011-2277-2

[3] Kouzma NM, Kennedy GA (2002) Homework, stress, and mood disturbance in senior high school students. Psychol Rep 91: 193-198.

http://www.ncbi.nlm.nih.gov/pubmed/12353781

[4] Ebrecht M, Hextall J, Kirtley LG, Taylor A, Dyson M, et al. (2004) Perceived stress and cortisol levels predict speed of wound healing in healthy male adults. Psychoneuroendocrinology 29: 798809. http://www.ncbi.nlm.nih.gov/pubmed/15110929 
[5] Vileikyte L (2005) Stress and wound healing. Clin Dermatol 25: 49-55.

http://www.sciencedirect.com/science/article/pii/S0738081X06001362

[6] Johnstone SE, Baylin SB (2010) Stress and the epigenetic landscape: a link to the pathobiology of human diseases? Nat Rev Genet 11: 806-12.

http://www.nature.com/nrg/journal/v11/n11/abs/nrg2881.html

[7] Bjelland I, Dahl AA, Haug TT, Neckelmann D (2002) The validity of the Hospital Anxiety and Depression Scale. An updated literature review. J Psychosom Res 52: 69-77. http://www.ncbi.nlm.nih.gov/pubmed/11832252

[8] McAlonan GM, Lee AM, Cheung V, Cheung C, Tsang KW, et al. (2007) Immediate and sustained psychological impact of an emerging infectious disease outbreak on health care workers. Can J Psychiatry 52: 241-247.

http://www.ncbi.nlm.nih.gov/pubmed/17500305

[9] Bieling PJ, Antony MM, Swinson RP (1998) The State-Trait Anxiety Inventory, Trait version: structure and content re-examined. Behav Res Ther 36: 777-788.

http://www.ncbi.nlm.nih.gov/pubmed/9682533

[10] Jackson C (2007) The General Health Questionnaire. Occup Med 57: 79. http://occmed.oxfordjournals.org/content/57/1/79.full

[11] Butler G (1993) Definitions of stress. Occas Pap R Coll Gen Pract 61: 1-5. http://www.ncbi.nlm.nih.gov/pmc/articles/PMC2560943/

[12] Jex SM, Beehr TA, Roberts CK (1992) The meaning of occupational stress items to survey respondents. J Appl Psychol 77: 623-628. http://www.ncbi.nlm.nih.gov/pubmed/1429346 
[13] Karlen J, Ludvigsson J, Frostell A, Theodorsson E, Faresjo T (2011) Cortisol in hair measured in young adults - a biomarker of major life stressors? BMC Clin Pathol 11: 1-12. http://www.biomedcentral.com/1472-6890/11/12

[14] Cohen S, Doyle WJ, Skoner DP (1999) Psychological stress, cytokine production, and severity of upper respiratory illness. Psychosom Med 61: 175-180.

http://www.ncbi.nlm.nih.gov/pubmed/10204970

[15] Rief W, Mills PJ, Ancoli-Israel S, Ziegler MG, Pung MA, et al. (2010) Overnight changes of immune parameters and catecholamines are associated with mood and stress. Psychosom Med 72: 755-762. http://www.ncbi.nlm.nih.gov/pubmed/20841563

[16] Van Eck M, Berkhof H, Nicolson N, Sulon J (1996) The effects of perceived stress, traits, mood states, and stressful daily events on salivary cortisol. Psychosom Med 58: 447-458. http://www.ncbi.nlm.nih.gov/pubmed/8902896

[17] Volkmann ER, Weekes NY (2006) Basal SIgA and cortisol levels predict stress-related health outcomes. Stress and Health 22: 11-23. http://onlinelibrary.wiley.com/doi/10.1002/smi.1077/abstract

[18] Cohen S, Kamarck T, Mermelstein R (1983) A global measure of perceived stress. J Health Soc Behav 24: 385-396. http://psycnet.apa.org/psycinfo/1984-24885-001

[19] Al kalaldeh MT, Abu Shosha GM (2012) Application of the perceived stress scale in health care studies. An analysis of literature. Int J Acad Res 4: 45-50.

http://connection.ebscohost.com/c/articles/80212926/application-perceived-stress-scale-healthcare-studies-analysis-literature 
[20] Leon KA, Hyre AD, Ompad D, Desalvo KB, Muntner P (2007) Perceived stress among a workforce 6 months following hurricane Katrina. Soc Psychiatry Psychiatr Epidemiol 42: 10051011.

[21] Shah M, Hasan S, Malik S, Sreeramareddy CT (2010) Perceived stress, sources and severity of stress among medical undergraduates in a Pakistani medical school. BMC Med Educ 10:2. http://www.biomedcentral.com/1472-6920/10/2

[22] Lane JD, Seskevich JE, Pieper CF (2007) Brief meditation training can improve perceived stress and negative mood. Altern Ther Health Med 13: 38-44.

http://www.ncbi.nlm.nih.gov/pubmed/17283740

[23] Golden-Kreutz DM, Thornton LM, Wells-Di Gregorio S, Frierson GM, Jim HS, et al. (2005) Traumatic stress, perceived global stress, and life events: prospectively predicting quality of life in breast cancer patients. Health Psychol 24: 288-296.

http://www.ncbi.nlm.nih.gov/pubmed/15898865

[24] Sims R, Gordon S, Garcia W, Clark E, Monye D, et al. (2008) Perceived stress and eating behaviors in a community-based sample of African Americans. Eat Behav 9: 137-142. http://www.ncbi.nlm.nih.gov/pmc/articles/PMC2562737/

[25] Dickerson SS, Kemeny ME (2004) Acute stressors and cortisol responses: a theoretical integration and synthesis of laboratory research. Psychol Bull 130: 355-91.

http://www.ncbi.nlm.nih.gov/pubmed/15122924

[26] Axelrod J, Reisine TD (1984) Stress hormones: their interaction and regulation. Science 224: 452-9. http://www.ncbi.nlm.nih.gov/pubmed/6143403 
[27] Dimsdale JE, Moss J (1980) Plasma catecholamines in stress and exercise. Jama 243: 340-2. http://www.ncbi.nlm.nih.gov/pubmed/7351746

[28] Nater UM, Skoluda N, Strahler J (2013) Biomarkers of stress in behavioural medicine. Curr Opin Psychiatry 26: 440-5. http://www.ncbi.nlm.nih.gov/pubmed/23867656

[29] Heim C, Newport DJ, Heit S, Graham YP, Wilcox M, et al. (2000) Pituitary-adrenal and autonomic responses to stress in women after sexual and physical abuse in childhood. Jama 284: 592-597. http://www.ncbi.nlm.nih.gov/pubmed/10918705

[30] Tomei F, Rosati MV, Baccolo TP, Bernardini A, Ciarrocca M, et al. (2003) Plasma concentration of adrenocorticotropic hormone in traffic policemen. J Occup Health 45: 242-247. http://www.ncbi.nlm.nih.gov/pubmed/14646285

[31] Malarkey WB, Pearl DK, Demers LM, Kiecolt-Glaser JK, Glaser R (1995) Influence of academic stress and season on 24-hour mean concentrations of ACTH, cortisol, and betaendorphin. Psychoneuroendocrinology 20: 499-508.

http://www.ncbi.nlm.nih.gov/pubmed/7675934

[32] Schulz P, Kirschbaum C, Prüßner J, Hellhammer D (1998) Increased free cortisol secretion after awakening in chronically stressed individuals due to work overload. Stress Med 14: 91-97. http://onlinelibrary.wiley.com/doi/10.1002/\%28SICI\%291099-

1700\%28199804\%2914:2\%3C91::AID-SMI765\%3E3.0.CO;2-S/abstract

[33] Peeters F, Nicolson NA, Berkhof J (2004) Levels and variability of daily life cortisol secretion in major depression. Psychiatry Res 126: 1-13.

http://www.ncbi.nlm.nih.gov/pubmed/15081622 
[34] Zarkovic M, Stefanova E, Ciric J, Penezic Z, Kostic V (2003) Prolonged psychological stress suppresses cortisol secretion. Clin Endocrinol 59: 811-6.

http://www.ncbi.nlm.nih.gov/pubmed/14974927

[35] Halter JB, Beard JC, Porte D Jr (1984) Islet function and stress hyperglycemia: plasma glucose and epinephrine interaction. Am J Physiol 247: E47-52.

http://www.ncbi.nlm.nih.gov/pubmed/6377920

[36] Porte D Jr, Williams RH (1966) Inhibition of insulin release by norepinephrine in man. Science 152: 1248-50. http://www.ncbi.nlm.nih.gov/pubmed/5327883

[37] Genuth SM (1973) Plasma insulin and glucose profiles in normal, obese, and diabetic persons. Ann Intern Med 79: 812-822. http://www.ncbi.nlm.nih.gov/pubmed/4761908 


\section{Chapter 6}

Conclusions and Future Directions 
Many topics have been addressed in this dissertation, with an overarching goal to build on the present knowledge of the biochemical adaptations that take place to protect and maintain optimal function. This document communicates some of the vital aspects of investigating biochemical markers of stress, such as consideration of all contributing types of stressors, localized versus systemic responses, biological matrices, and analysis of complex biological datasets. Decades of research exploring the mechanisms that drive biological processes has uncovered crucial information that serves to enhance our understanding of the pathogenesis and exacerbation of diseases, disorders, exposures, or general perturbations. All of this work is done to ultimately serve the general public through development of treatments, validation of diagnostic biomarkers, and enhancement of overall health. The research in this field is expected to continue and grow as there are still many challenges that prevent a comprehensive understanding of various features of the stress response.

While there are many factors that can be addressed, the challenges of timing and data analysis are two major issues to focus on for future work. One of the most difficult variables to work with when measuring biological responses is time. Chapter 2 briefly touches on the temporal assessment of a response, but there is no way to confirm that the most significant time points were captured. Ideally, adding more time points would provide the best depiction of the fluctuations in biochemical response over time, but in many cases, it is impractical to do so because the number of samples and cost would dramatically increase. As a result, further work in determining the optimum timing of sample collection is an essential component of making advancements in this field. 
Another prominent obstacle lies in the analysis of data. The need for robust statistical analysis methods to handle complex biological data has been acknowledged with the creation of software programs, algorithms, and modeling techniques. However, there are still limitations to the current methods, prompting more bioinformatics research. The issue in proposing new analysis approaches is that it takes a great deal of testing to validate the viability and robustness of the method. In the case of using network centrality parameter analysis (demonstrated in Chapter 3) to examine a large number of variables, many more datasets must be tested to explore all potential capabilities, as well as any weaknesses of the approach. 


\section{APPENDIX A: CYTOKINE CALIBRATION CURVES}

IL-1 $\alpha$

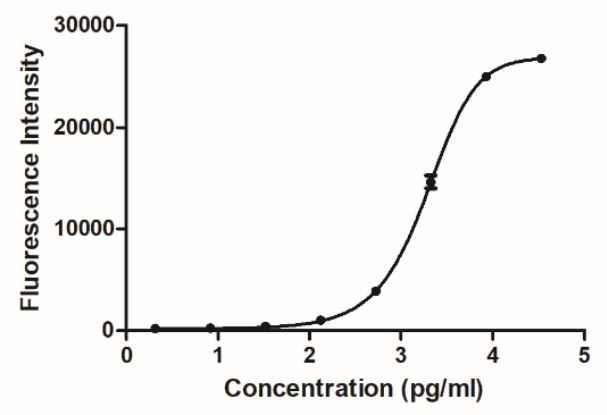

IL-2

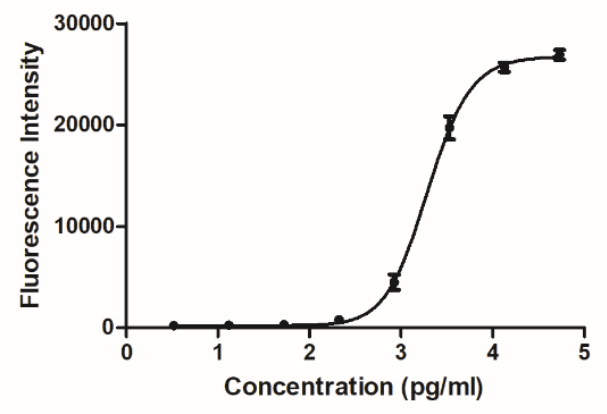

MIP-1 $\alpha$

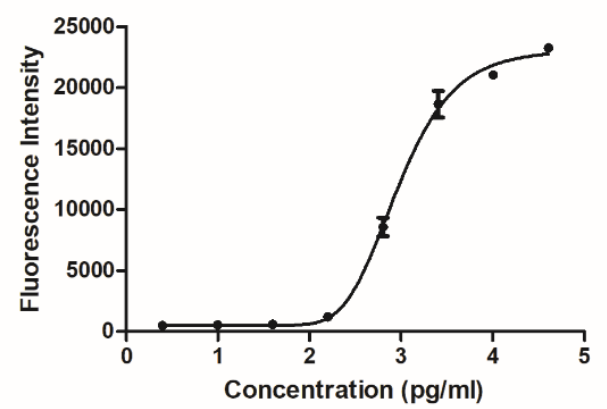

IL-1 $\beta$

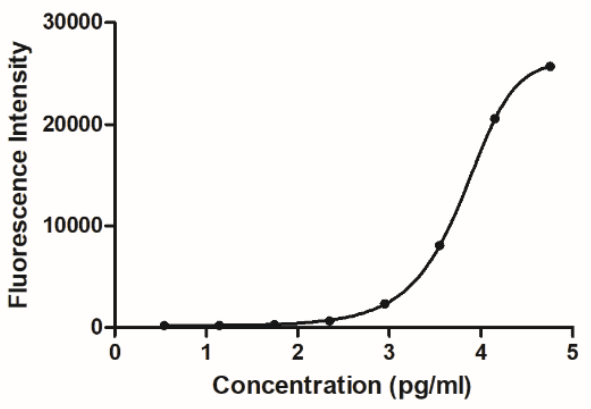

IL-6

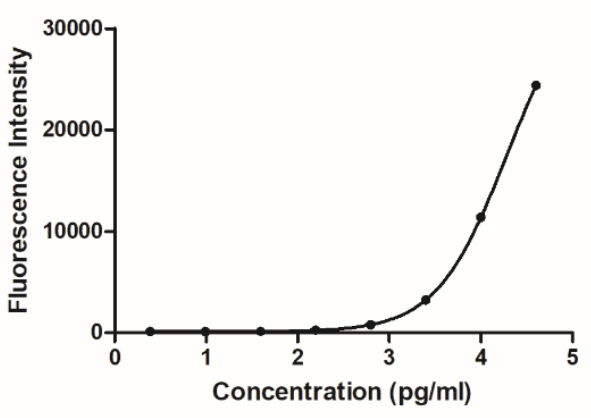

TNF- $\alpha$

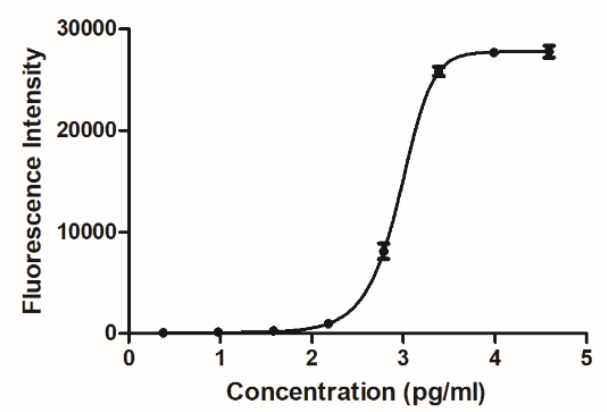




\section{APPENDIX B1: CHAPTER 4 SUPPLEMENTARY TABLES AND FIGURES}

\section{Normalized Cytokine Concentration}
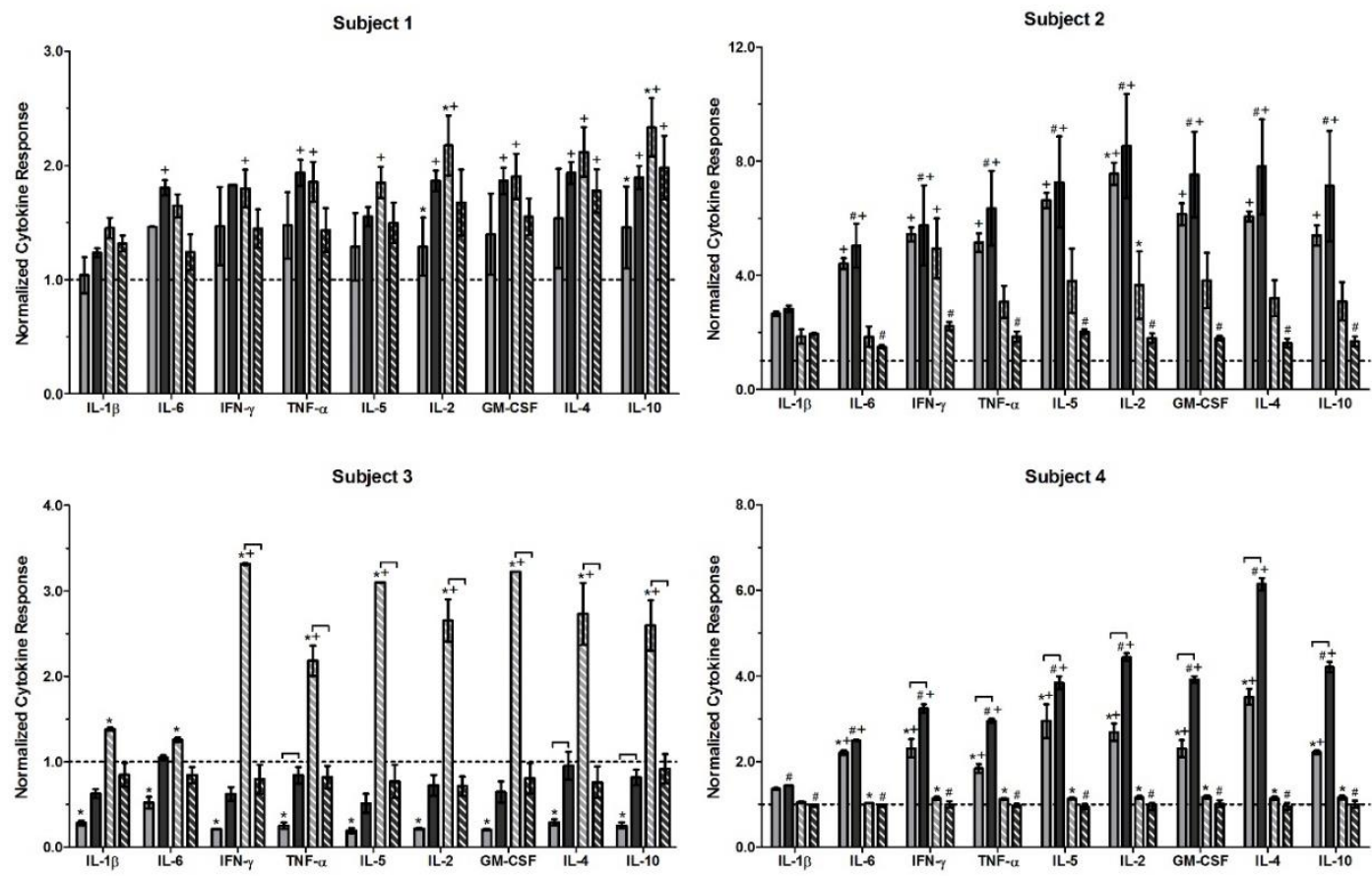

Figure B1. Subjects 1-4: Normalized cytokine response following team and individual performance. Salivary cytokine (IL-1 $\beta$, IL-6, IFN- $\gamma$, TNF- $\alpha$, IL-5, IL-2, GM-CSF, IL-4, and IL10) levels were determined for subjects 1-4 after experiencing success and failure as an individual and as a team. All responses were normalized to baseline concentrations measured prior to beginning the sessions. Error bars reflect \pm standard error of the mean due to the variation in sample replicates $(\mathrm{N}=3)$. Statistically significant differences between individual and team responses are marked with a matching symbols $\left(^{*}\right)$ and (\#). Significant differences between success and failure associated responses are notated with brackets. + indicates a significant change, compared to the baseline (dashed line indicates normalized response $=1$ ). Statistically significant $(\mathrm{p}<0.05)$ differences were determined by 2-way ANOVA with a Bonferroni post-test. 


\section{APPENDIX B2: CHAPTER 4 SUPPLEMENTARY TABLES AND FIGURES}

\section{Normalized Cytokine Concentration}
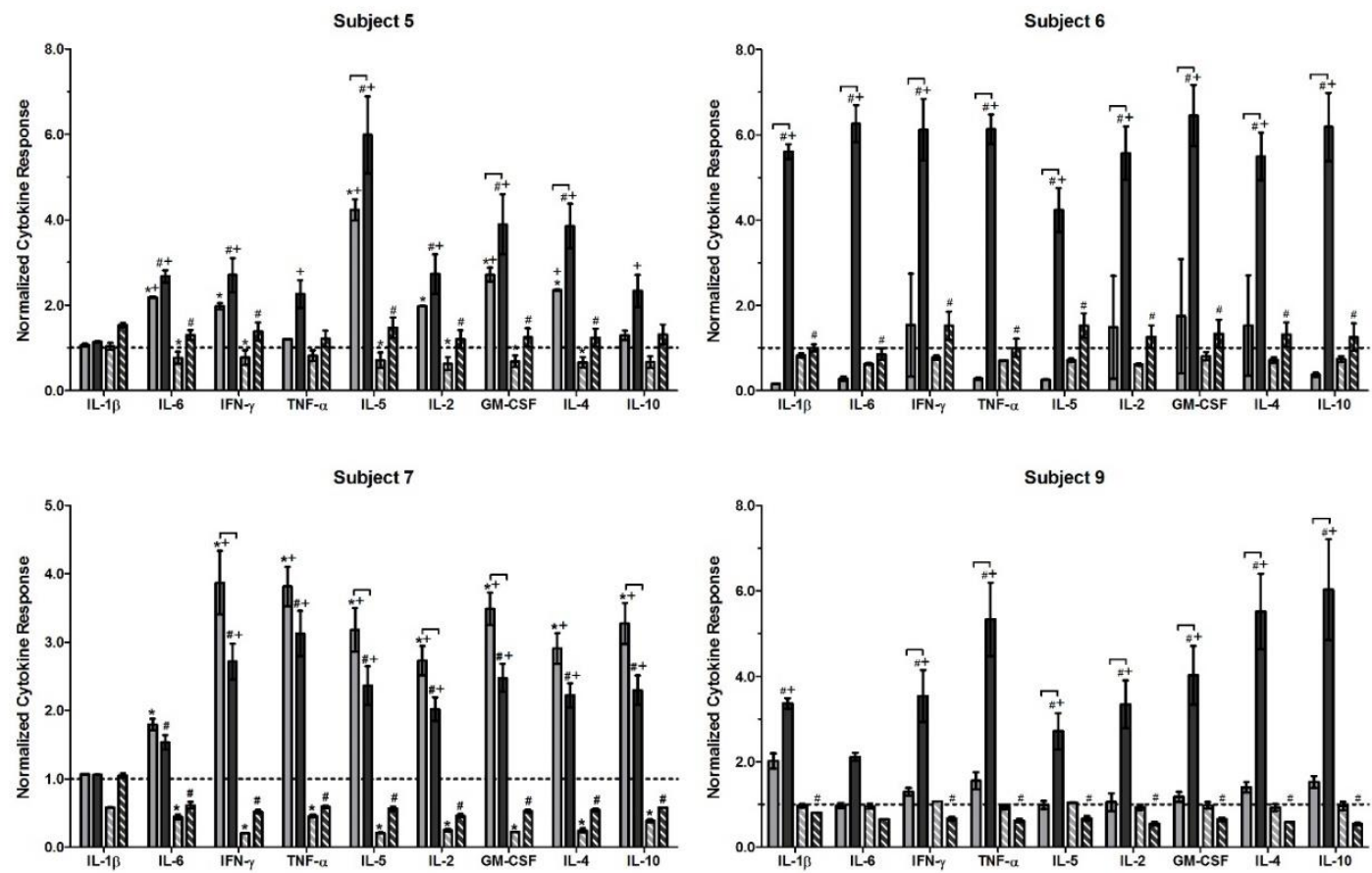

Figure B2. Subjects 5-8: Normalized cytokine response following team and individual performance. Salivary cytokine (IL-1 $\beta$, IL-6, IFN- $\gamma$, TNF- $\alpha$, IL-5, IL-2, GM-CSF, IL-4, and IL10) levels were determined for subjects 5-8 after experiencing success and failure as an individual and as a team. All responses were normalized to baseline concentrations measured prior to beginning the sessions. Error bars reflect \pm standard error of the mean due to the variation in sample replicates $(\mathrm{N}=3)$. Statistically significant differences between individual and team responses are marked with a matching symbols (*) and (\#). Significant differences between success and failure associated responses are notated with brackets. + indicates a significant change, compared to the baseline (dashed line indicates normalized response $=1$ ). Statistically significant $(\mathrm{p}<0.05)$ differences were determined by 2-way ANOVA with a Bonferroni post-test. 


\section{APPENDIX B3: CHAPTER 4 SUPPLEMENTARY TABLES AND FIGURES}

\section{Normalized Cytokine Concentration}
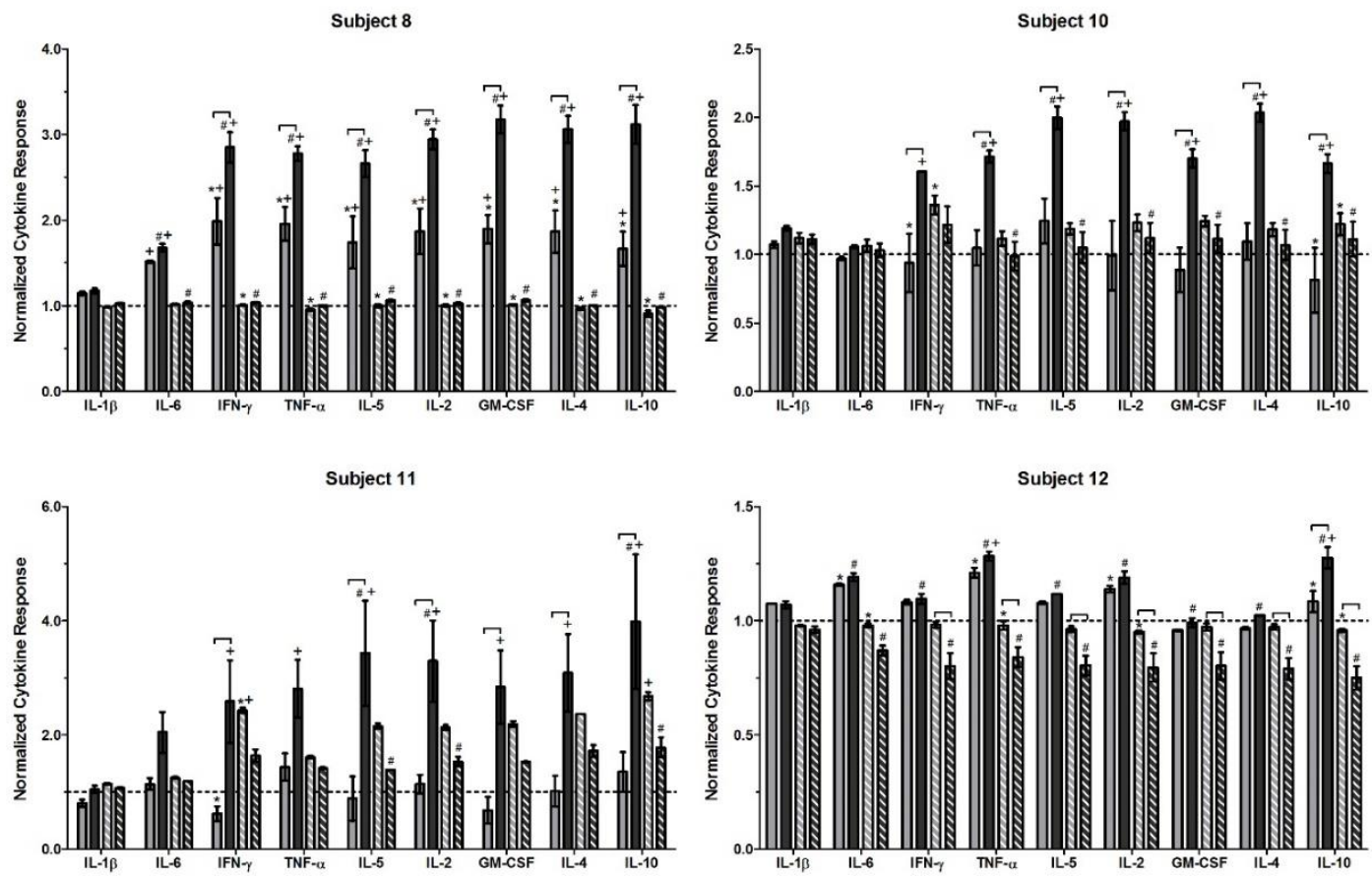

Figure B3. Subjects 9-12: Normalized cytokine response following team and individual performance. Salivary cytokine (IL-1 $\beta$, IL-6, IFN- $\gamma$, TNF- $\alpha$, IL-5, IL-2, GM-CSF, IL-4, and IL10) levels were determined for subjects 9-12 after experiencing success and failure as an individual and as a team. All responses were normalized to baseline concentrations measured prior to beginning the sessions. Error bars reflect \pm standard error of the mean due to the variation in sample replicates $(\mathrm{N}=3)$. Statistically significant differences between individual and team responses are marked with a matching symbols (*) and (\#). Significant differences between success and failure associated responses are notated with brackets. + indicates a significant change, compared to the baseline (dashed line indicates normalized response $=1$ ). Statistically significant $(\mathrm{p}<0.05)$ differences were determined by 2-way ANOVA with a Bonferroni post-test. 


\section{Normalized Cytokine Concentration}
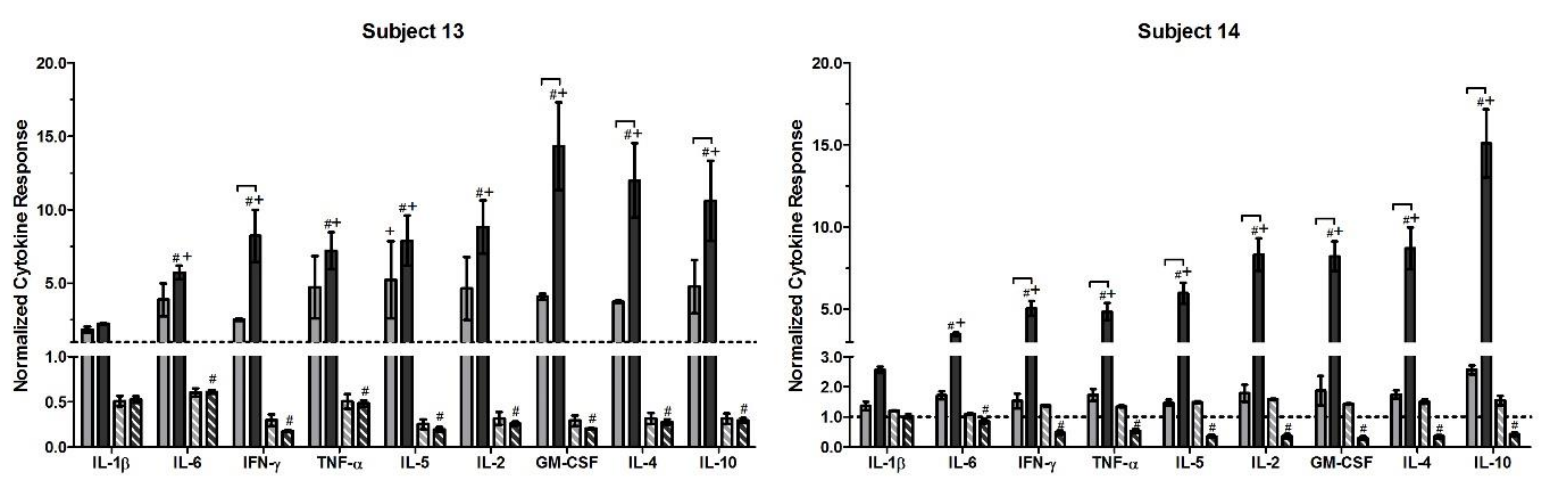

Figure B4. Subjects 13-14: Normalized cytokine response following team and individual performance. Salivary cytokine (IL-1 $\beta$, IL-6, IFN- $\gamma$, TNF- $\alpha$, IL-5, IL-2, GM-CSF, IL-4, and IL10) levels were determined for subjects 13-14 after experiencing success and failure as an individual and as a team. All responses were normalized to baseline concentrations measured prior to beginning the sessions. Error bars reflect \pm standard error of the mean due to the variation in sample replicates $(\mathrm{N}=3)$. Statistically significant differences between individual and team responses are marked with a matching symbols $(*)$ and (\#). Significant differences between success and failure associated responses are notated with brackets. + indicates a significant change, compared to the baseline (dashed line indicates normalized response $=1$ ). Statistically significant $(\mathrm{p}<0.05)$ differences were determined by 2-way ANOVA with a Bonferroni post-test. 